Portland State University

PDXScholar

Winter 4-4-2017

\title{
An IR and RF Based System for Functional Gait Analysis in a Multi-Resident Smart-Home
}

Erich Reinhardt Schafermeyer

Portland State University

Follow this and additional works at: https://pdxscholar.library.pdx.edu/open_access_etds

Part of the Electrical and Computer Engineering Commons Let us know how access to this document benefits you.

\section{Recommended Citation}

Schafermeyer, Erich Reinhardt, "An IR and RF Based System for Functional Gait Analysis in a MultiResident Smart-Home" (2017). Dissertations and Theses. Paper 3502.

https://doi.org/10.15760/etd.5386

This Thesis is brought to you for free and open access. It has been accepted for inclusion in Dissertations and Theses by an authorized administrator of PDXScholar. Please contact us if we can make this document more accessible: pdxscholar@pdx.edu. 
An IR and RF Based System for Functional Gait Analysis

in a Multi-Resident Smart-Home

by

Erich Reinhardt Schafermeyer

A thesis submitted in partial fulfillment of the requirements for the degree of

\author{
Master of Science \\ in \\ Electrical and Computer Engineering
}

Thesis Committee:

Eric Wan, Chair

Melanie Mitchel

James McNames

Portland State University

2017 


\begin{abstract}
Changes in the gait characteristics, such as walking speed and stride length, of a person living at home can be used to presage cognitive decline, predict fall potential, monitor long-term changes in cognitive impairment, test drug regimens, and more. This thesis presents a novel approach to gait analysis in a smart-home environment by leveraging new advances in inexpensive sensors and embedded systems to create novel solutions for in-home gait analysis. Using a simple, non-invasive hardware system consisting entirely of wall-mounted infrared and radio frequency sensor arrays, data is collected on the gait of subjects as they pass by. This data is then analyzed and sent to a clinician for further study. The system is non-invasive in that it does not use cameras and could be built into the molding of a home so that it would be nearly invisible. In a finished prototype version, the system presented in this thesis could be used to analyze the gait characteristics of one or more subjects living in a home environment while ignoring the data of visitors and other non-subject cohabitants. The ability to constantly collect data from a home environment could provide thousands of observations per year for clinical analysis. Providing such a robust data set may allow people with gait impairment to live at home longer and more safely before transitioning to a care facility, have a reduced fall risk due to better prediction, and live a healthier life in old age.
\end{abstract}


To Mary Beth Llorens and Howard Newman, my parents, who have always encouraged and supported me in finding my own path. Thank you. 


\section{Acknowledgements}

I would like to acknowledge the many people who contributed to the research documented in this thesis. Dr. Eric Wan was my thesis advisor, primary investigator, and boss who saw me through this process from beginning to end. Fatima Adenwala, Shadman Samin, Tanisha Payne, Colin Doolittle, Jamie Wong, Noah Zentzis, Walter Woods, and the many other student contributors in the lab were invaluable. Dr. Peter Jacobs, Jon Folsom, John Condon, David Edwards, Anindya Paul, Nick Preiser, and the many people at EmbedRF got me off to a great start. Dr. James McNames, Dr. Melanie Mitchel, Dr. Bob Bass, Mark Faust, Dean Ren Su, and the many faculty members facilitated my learning and growing as an engineer while at Portland State University.

This work could not have been completed without the hardware development skills of Shadman Samin, with whom I worked on all aspects of the data collection hardware. Shadman is directly responsible for prototypes 1 and 3 (the next one) of the gait analysis system. He is a longtime contributor to the lab and a valuable asset to the university. Tanisha Payne, Eric Wan, Jon Condon Fatima Adenwala, and Shadman Samin were all instrumental in the data collection process. Each often spent hours of his or her weekend in the lab walking back and forth, running data collections, hauling hardware to and from a data collection site, and debugging the many aspects of the system.

Hardware development was greatly aided by the PCB design skills of Jon Folsom who taught me to design PCBs correctly. I would like to thank him for his patience and 
expertise in hardware and circuit design and debugging. The data collection software for the subject ID systems was designed and developed by Nick Preiser at OHSU/EmbedRF, who is a wizard. RF Sensor firmware was originally written by Peter Jacobs and further developed by Jon Folsom.

All of the algorithms, digital signal processing software, and data flow designs were done under the advisement of Eric Wan and Anindya Paul who were instrumental in helping me to understand the complex inner workings of the many statistical methods attempted in the pursuit of this research. Similarly, all credit for machine learning understanding goes to Melanie Mitchel and Eric Wan for helping in the design, debugging, implementation and analysis of the machine learning algorithms used herein.

Thanks also to Dean Ren Su for making graduate school more fun and being a wonderful human. Thank you all for an interesting, trying, fun, hard, detailed, painstaking, rewarding experience. It has been a pleasure. You guys rock, I couldn't have done it without you. 


\section{Table of Contents}

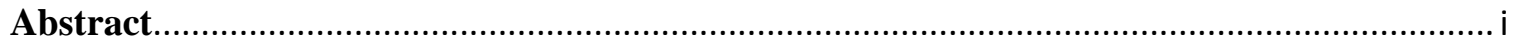

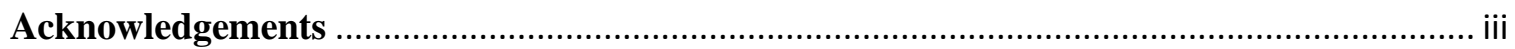

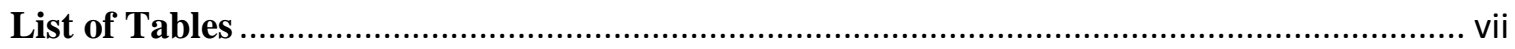

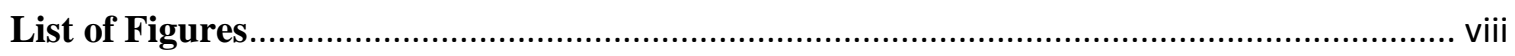

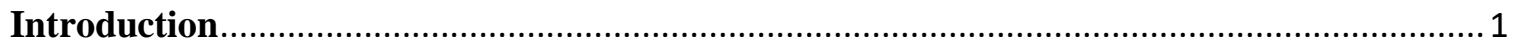

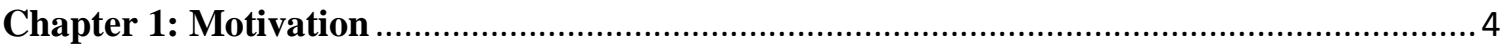

Aging, Cognitive Decline, and the American Healthcare System ...................................... 4

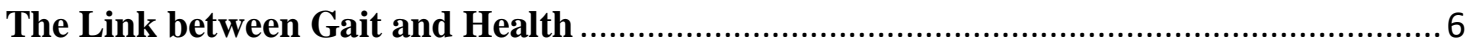

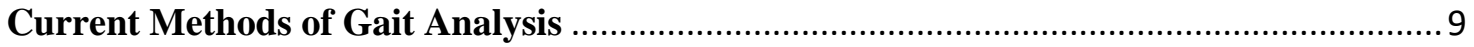

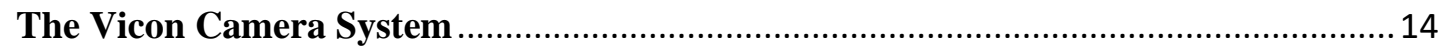

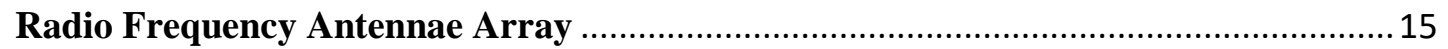

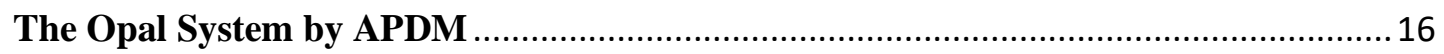

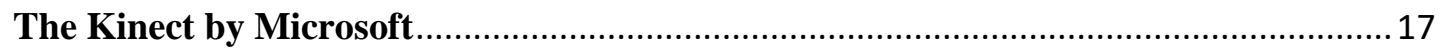

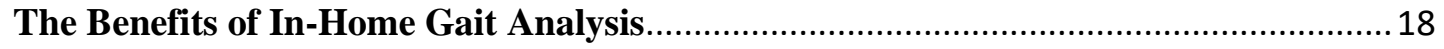

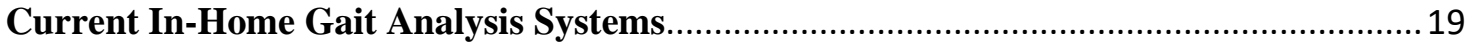

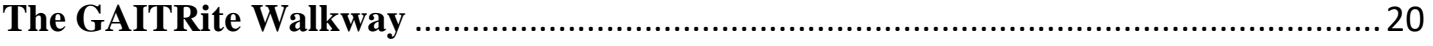

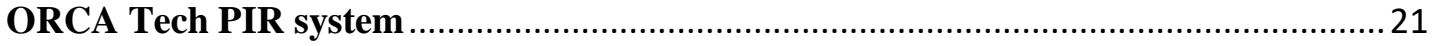

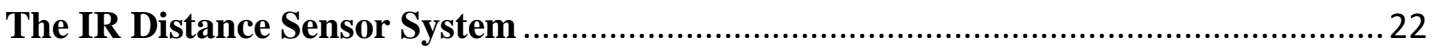

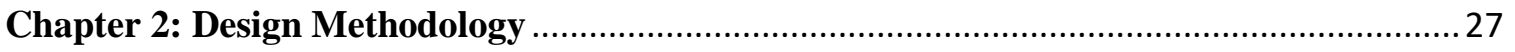

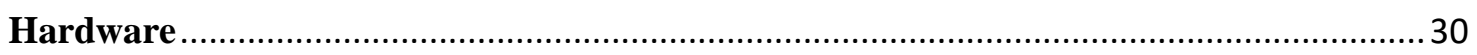

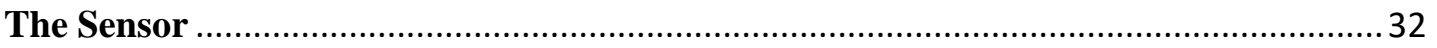

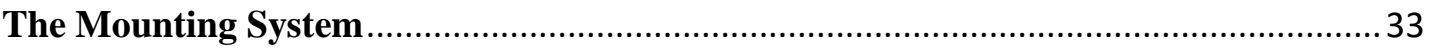

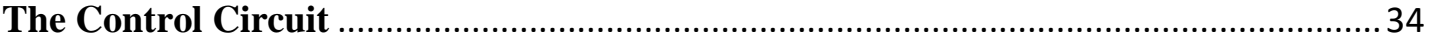

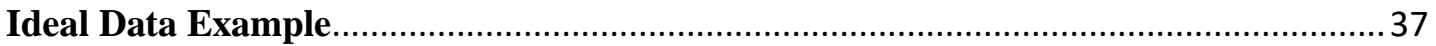

Software

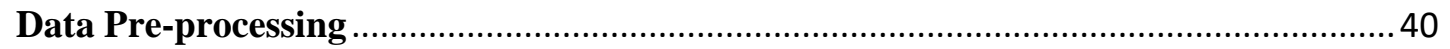

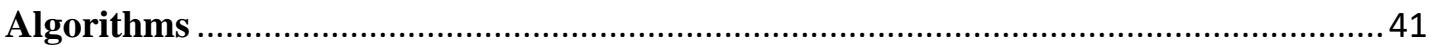

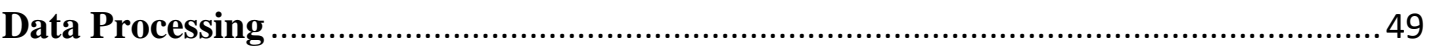




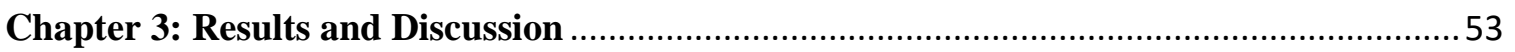

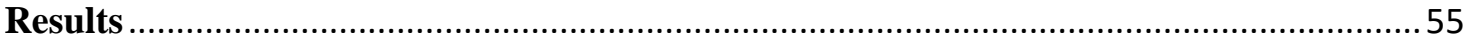

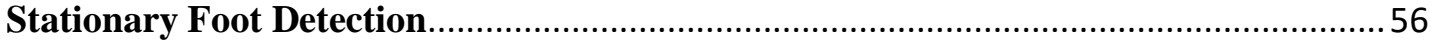

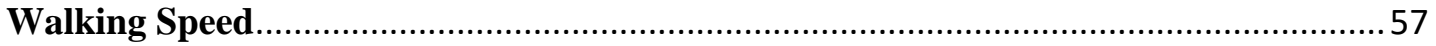

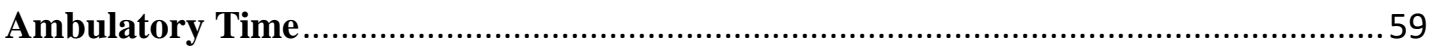

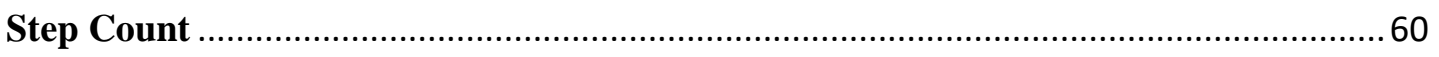

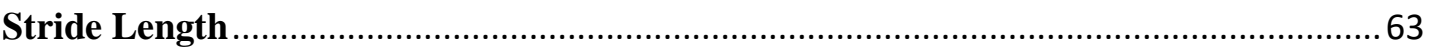

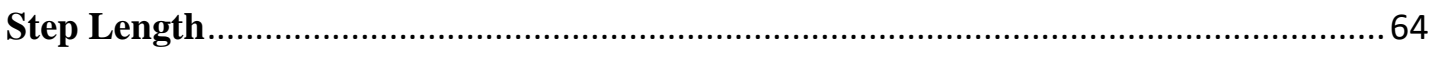

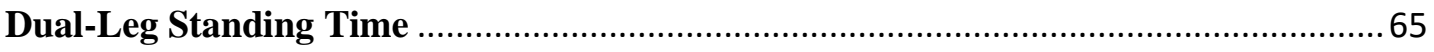

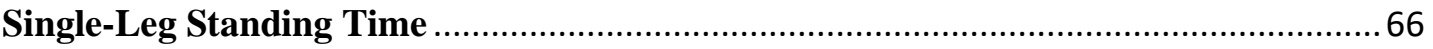

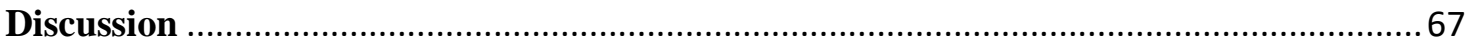

Chapter 4: Subject Identification using IR and RF Fingerprinting ................................... 71

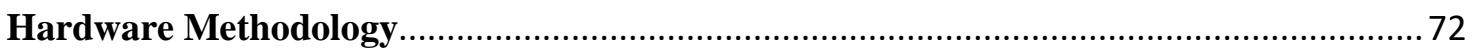

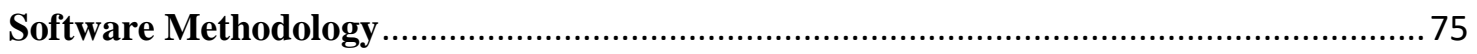

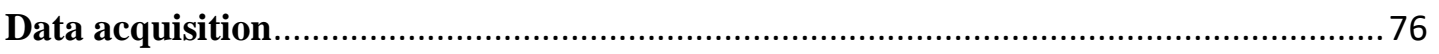

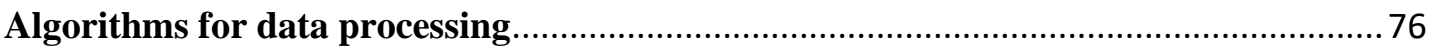

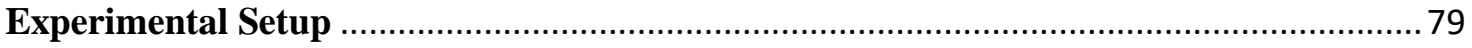

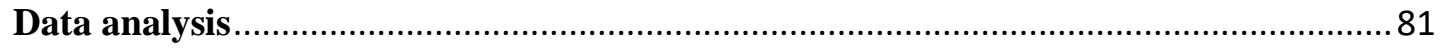

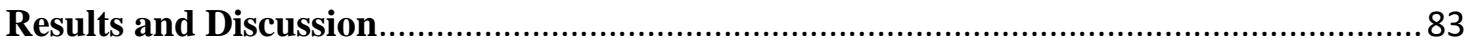

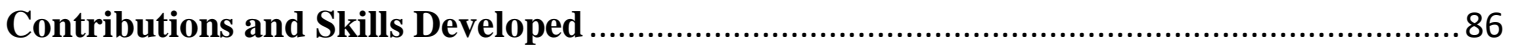

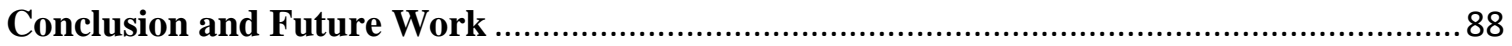

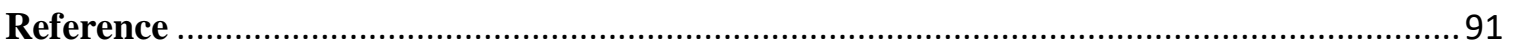

Appendix: Algorithms for Gait Analysis and Subject ID ............................................. 94

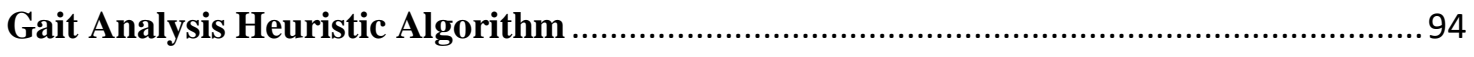

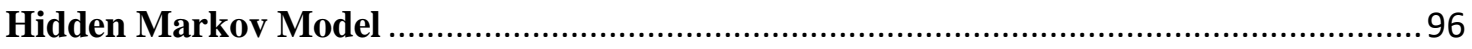




\section{List of Tables}

TABLE 1: A REVIEW OF CHANGES IN GAIT METRICS OVER TIME USING THE GAITRITE WALKWAY SYSTEM................................................................................................ 9

TABle 2: Desired ACCURACy For a GaIT ANALysis System. 'LOWEST' COLUMN DEPICTS LOWEST NECESSARY ACCURACY, 'HIGHEST' COLUMN DEPICTS HIGHEST..................... 27

TABLE 3: RMSE TABLE. GAIT METRICS BY ROW, TRACKING ALGORITHM BY COLUMN. Algorithms include Hidden Markov Model (HMM), Heuristic Model (HEURISTIC), AND THE APF MODEL (PARTICLE FILTER).

TABLE 4: NORMALIZED RMSE VALUES (NRMSE). GAIT METRICS BY ROW, TRACKING ALGORITHM BY COLUMN. AlgorithmS INCLUde Hidden MARKov Model (HMM), Heuristic Model (Heuristic), AND the APF model (PARTICle Filter). 54

TABle 5: Percentage of DATA USED in Each CALCUlation. Gait METRics By ROW, TRACKING ALGORITHM BY COLUMN. 55

TABLE 6: A SUMMARY OF GAIT METRICS, THEIR LOWEST DESIRABLE ACCURACY, AND ACHIEVED RESULTS.

TABLE 7: CONFUSION MATRIX OF THREE SUBJECTS (A-FEMALE, B-MALE, C-MALE) CLASSIFIED USING A GMM TRAINED ON ALL AVAILABLE LABELED OBSERVATIONS. 5FOLD DAILY CROSS VALIDATION.

TABLE 8: CONFUSION MATRIX OF THREE SUBJECTS (A-FEMALE, B-MALE, C-MALE) CLASSIFIED USING A GMM TRAINED ON APPROXIMATELY 140 LABELED OBSERVATIONS (6\% OF DATA) PER SUBJECT. 5-FOLD DAILY CROSS VALIDATION WITH MONTE-CARLO SAMPLING 


\section{List of Figures}

FIGURE 1: REPRODUCTION OF A FIGURE FROM FUKUI ET AL. THE DISTANCE BETWEEN PEAKS IN THE LOWER FIGURE GIVES DISTANCE AND THE BREADTH OF THE PLATEAU ASSOCIATED WITH SENSOR 5 IN THE TOP FIGURE GIVES TIMING. ................................ 25

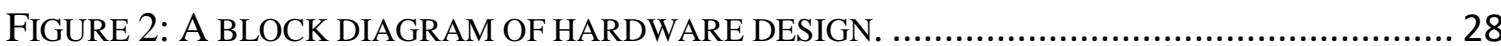

FIGURE 3: THE SECOND PROTOTYPE OF THE IR SENSOR ARRAY. LEFT IMAGE DEPICTS AN ARRAY OF 24 SENSORS IN A SIMULATED HALLWAY SETUP IN THE BSP LAB. RIGHT IS A DETAIL SHOWING THE SENSOR IN AN ACRYLIC HOUSING ATTACHED TO EXTRUDED

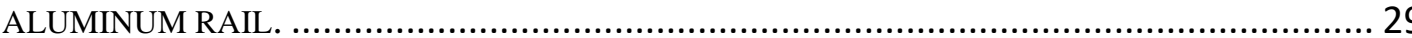

FIGURE 4: REPRODUCED FROM THE SHARP GP2Y0A60SZXF_E INFRARED SENSOR SPECIFICATION SHEET. THIS IMAGE DEPICTS THE SENSOR LAYOUT, .............................. 32

FIGURE 5: REPRODUCTION OF A FIGURE DEPICTING 80/20 1 INCH EXTRUDED ALUMINUM RAILING.

FIGURE 6: CONTROL CIRCUIT BOARD.12 IR SENSORS ATTACHMENT POINTS WITH SHUNT CIRCUITS ENTERING A SINGLE LINE FEMALE PIN SET WHERE A DAUGHTER BOARD IS ATTACHED CARRYING A MUX

FIGURE 7: AN INTUITIVE LOOK AT THE WAVEFORMS RESULTING FROM A WALK ACROSS 12 SENSORS (HALF THE NUMBER ACTUALLY BEING USED). THE FIGURE TO THE TOP LEFT ASSOCIATES' STEP PATTERNS WITH WAVEFORMS WHILE THE FIGURE TO THE TOP RIGHT SHOWS THE RESULTING WALK ACROSS ALL LINKS. LOWER FIGURES SHOW REAL DATA AS HEAT MAP (LEFT) AND TRACES (RIGHT).

FIGURE 8: FLOW CHART OF THE DATA PROCESSING WORKFLOW. DATA PROCESSING BEGINS WHERE DATA ACQUISITION ENDS AND PROCEEDS UNTIL RELEVANT GAIT METRICS ARE EXTRACTED FOR ANALYSIS BY A CLINICIAN. 38

FIGURE 9: A VISUALIZATION OF MEASUREMENT MODEL OUTPUT BASED UPON UNDERLYING FOOT POSITION FOR BOTH FEET. NOTE THAT THE NEAR FOOT IS THE TALLER WAVEFORM, BEGINNING AT $0.5 \mathrm{~S}$ WHILE THE FAR FOOT IS THE SHORTER WAVEFORM BEGINNING AROUND 1S

FIGURE 10: THE THREE STAGES OF DATA ASSOCIATION: (A) TRACKING THE NEAR FOOT, (B) SEPARATING DATA WINDOWED ABOUT THE ESTIMATED NEAR FOOT POSITION FROM ALL OTHER DATA, (C) TRACKING THE FAR FOOT NOW THAT THE NEAR FOOT IS REMOVED. ALSO THE CREATION OF A PRIOR OCCURS. (D) TRACKING THE FAR FOOT (RED) WITH EXPERT LABELING (GREEN) AND PRIOR BASED UPON NEAR FOOT POSITION (PURPLE). 47

FIGURE 11: AN EXAMPLE OF FAR FOOT PRIOR INFORMATION (PURPLE) OVERLAID ON SILHOUETTE-CANCELLED DATA. ALSO PICTURED: TRACKED FOOT (RED), GROUND TRUTH (GREEN). 
Figure 12: ScAtTER Plot OF WALKING SPEED (WS). TRUE WS (AS DETERMinED By GR MAT) IS ON THE X-AXIS, WHILE ESTIMATED IS ON Y-AXIS. THUS, THE CLOSER TO THE DIAGONAL (RED) LINE THE BETTER. .......................................................... 56

Figure 13: ScAtTER Plot OF AMbULATORY Times (AT). TRUE AT (AS DETERMined By GR MAT) IS ON THE X-AXIS, WHILE ESTIMATED IS ON Y-AXIS. THUS, THE CLOSER TO THE DIAGONAL (RED) LINE THE BETTER.

FIGURE 14: BOXPLOT OF STEP COUNT (SC) FOR THE HEURISTIC ALGORITHM. THE X-AXIS DEPICTS THE TRUE SC BASED ON GAITRITE MAT. THE Y-AXIS DEPICTS THE ESTIMATED STEP COUNT.

FIGURE 15: BOXPLOT OF STEP COUNT (SC) FOR HMM ALGORITHM. THE X-AXIS DEPICTS THE TRUE SC BASED ON GAITRITE MAT. THE Y-AXIS DEPICTS THE ESTIMATED STEP COUNT.

FIGURE 16: BOXPLOT OF STEP COUNT (SC) FOR THE APF. THE X-AXIS DEPICTS THE TRUE SC BASED ON GAITRITE MAT. THE Y-AXIS DEPICTS THE ESTIMATED STEP COUNT.

FIGURE 17: THIS FIGURE IS A REPRODUCTION OF A FIGURE FROM THE GAITRITE TECHNICAL LITERATURE. STEP LENGTH IS MEASURED FROM A TO L, STRIDE LENGTH IS MEASURED FROM A TO G, AND STRIDE WIDTH IS MEASURED FROM D TO L. 63

FIGURE 18: STRIDE LENGTH (STL). THE TRUE STL IS ON THE X-AXIS WHILE THE ESTIMATED STL IS ON THE Y-AXIS. 64

FiguRE 19: STEP LENGTH (SL). THE TRUE SL IS ON THE X-AXIS WHILE THE ESTIMATED SL IS ON THE Y-AXIS. 65

Figure 20: DUAL-LEG STANDING TIME (DST). THE TRUE DST IS ON THE X-AXIS WHILE THE ESTIMATED DST IS ON THE Y-AXIS. 66

Figure 21: Single-LEG STANDING TIME (SST). THE TRUE SST (AS DETERMINED BY THE GR) IS ON THE X-AXIS, WHILE THE ESTIMATED SST IS ON THE Y-AXIS. THE CLOSER TO THE DIAGONAL LINE THE BETTER.

FIGURE 22: ACCESS-POINTS (AP) TRANSCEIVERS ARE ARRANGED IN A HALLWAY. AS A PERSON WALKS PAST, THE RSS DISRUPTIONS BETWEEN LINKS ARE MEASURED AND USED TO RECOGNIZE THE PERSON. IR SENSORS PROVIDE TIMING INFORMATION TO EXTRACT WALKING SPEED.

FIGURE 23: EXAMPLE RSS WAVEFORMS FOR THREE DIFFERENT SUBJECTS . 73

FIGURE 24: PERCENT CLASSIFICATION ACCURACY VERSUS AMOUNT OF LABELED TRAINING DATA USED FOR BOTH A GMM AND FEED FORWARD NN. 83 


\section{Introduction}

America is aging, and as it does, new healthcare solutions are needed to serve this aging population. As baby boomers reach old age, defined as 65 and older, the burden on the healthcare system is dramatically increasing. One way to lessen the burden of an aging population on the healthcare system is to leverage innovative technologies to improve the efficiency of the system.

This thesis focuses on one such innovation: a simple, inexpensive in-home gait analysis device capable to providing real-time data to clinicians. Changes in gait metrics, such as walking speed and stride length, are associated with negative life events such as falls in persons with gait impairment. The accurate monitoring and analysis of gait characteristics over time can help a care provider diagnose and monitor change in a subject's health as well as the person's ability to live with little or no caregiver support. In addition, in-home gait monitoring can provide much more data to clinicians than clinical data alone. Such data can be used for fall-risk assessment and the monitoring of rehabilitation progress or the effects of a new medication.

The first part of the solution is a passive gait-analysis device. An array of 24 infrared (IR) sensors stationed along a hallway baseboard at $10.2 \mathrm{~cm}$ intervals collects data continuously. When a subject walks past, data is stored and analyzed using machine learning and signal processing techniques to extract relevant gait characteristics including ambulatory time, walking speed, stride length, step length, step count, single-support time, and dual-support time, with the possibility to expand to more metrics in the future, such as stride width and instantaneous foot velocity. 
The second part of the solution is a method for identifying individuals living together in a smart home. Take as an example an elderly man and woman living together at home. The man is diagnosed with mild cognitive impairment. Although there are a number of pre-existing solutions for monitoring the man at home, there are no ideal solutions for monitoring him independent of the woman's data points. To solve this problem, a passive, non-invasive subject-identification system consisting of four radiofrequency $(\mathrm{RF})$ transceivers and three IR transceivers placed in a doorway was created. Using machine learning and signal processing techniques, the unique IR/RF-fingerprint of each person can be determined after a very small number of training walks, in which it is known who is walking past the sensors. Subsequent walks can be classified as one of the two inhabitants with up to $99 \%$ accuracy depending on height and weight differences between the two people, and is capable of separating residents from non-residents. This system is inexpensive and can be added to existing homes.

The system is constructed using inexpensive, non-invasive sensors which the subject is not required to wear or carry. Additionally, it should cost less than 1,000 USD to produce, making it affordable for in-home installation. The system uses an array of IR sensors which detect the distance to an object passing within one meter of them. Data is acquired into software and analyzed using a stochastic signal processing technique called an Auxiliary Particle Filter (APF). The APF is made better by a number of other techniques including a data association technique called Sequential Silhouette Cancelation, a switch for keeping the far foot stationary when the near is known to be swinging, and a strong underlying model of how walking occurs used in conjunction with 
the process model. The system is capable of detecting step length with better than $10 \mathrm{~cm}$ accuracy, dual- and single-support times to within 170ms and 130ms respectively, and stride length to within $20.4 \mathrm{~cm}$. Additionally the system can separate a male and female subject with as much as $99 \%$ accuracy, allowing for the collection of gait data in a multiresident smart home environment. By combining these two technologies, a clinician will be able to track a person's gait characteristics with more comprehensive data than possible in a clinical setting. This innovative new technique for aging-in-place in a smarthome environment will help people to stay safely in the comfort of their own home far longer than currently possible. 


\section{Chapter 1: Motivation}

In 2016 assistive technologies for a smart home is a fledgling industry. Those solutions which do exist tend to be expensive, not automated for home use, or do not support the identification and separation of multiple inhabitants. This section will provide some background on the current state-of-the-art in in-home elderly care for persons with gait impairment.

\section{Aging, Cognitive Decline, and the American Healthcare System}

From the late 1940s through the early 1960s a baby boom was sparked by end of the last world war. By the middle of the 1960s the world's population had increased by 400 million people [1]. In 2016, the very first baby boomers are reaching old age. They join their predecessors in making up the 46 million Americans over the age of $65,14.5 \%$ of the total population, estimated to live in the US in 2014 [2]. By the year 2040 the cohort of Americans over the age of 65 is estimated by the US Census Bureau to more than double to 98 million people. An estimated 17.8 million senior citizens from that group will be over the age of 85 [3], which will be the average age of baby boomers in 2040.

With an aging population comes an increased healthcare cost. Fifteen percent of men and $24 \%$ of women over the age of 65 need some level of care ranging from inhome assistance to a full-time care staff at a nursing home. By the age of 85 these numbers increase dramatically to $46 \%$ for men and $62 \%$ for women with $15 \%$ and $25 \%$ 
respectively living in nursing homes [4]. A conservative estimate places the number of elderly people living in nursing homes in 2040 at around 6 million with one in three of those people over the age of 85 at a total annual cost of 326.2 billion dollars (2016 valuation). More than half the annual cost of nursing home care is incurred caring for those people 85 years and older.

A meta-analysis of previous studies which account for age ranges above 80 years by EL Schneider et al. [3] suggests that $51.5 \%$ of older Americans dwelling in care facilities suffer from some level of cognitive impairment. The same survey suggests that the rates of cognitive impairment increase dramatically from $2.8 \%$ at age 65 to $28 \%$ at ages 85 and above. As improved healthcare increases life expectancy of the oldest-old the rates of cognitive impairment will increase. Researchers in this field agree that agerelated statistics from the Social Security Administration and the US Census Bureau underestimate the lifespan of aging Americans. The underestimate is due to an unwillingness to project federal spending on healthcare research and biomedical improvements in the next 25 years and their effect on reduced mortality risk [1]. Based on a study by $\mathrm{Hu}$ and Cartwright [5], the cost of care in a nursing home is nearly double that of in-home care. Therefore changes in the number of elderly people in care facilities could have a huge impact on the US economy. If government estimates of longevity are negatively biased by 3.8 years, as suggested by SJ Olshansky et al. [1] the person-yearsof-life (a metric describing aggregate years across a population and abbreviated PYL) could increase by as much as 22 million PYL for those over the age of 85 by 2040. 
To help prevent the inevitable strain on social security in the US it would behoove researchers to try to limit the PYL of individuals in full-time care environments such as nursing homes. To achieve this end it is possible to leverage rapid improvements in computation, electronics and engineering. By creating systems which allow people in transitional phases of gait impairment to be closely monitored it is possible to keep them at home longer, thereby reduce expenditures on healthcare costs while simultaneously improving their quality of life. Systems which can help test drug regimens, monitor and report health concerns to doctors, and help alleviate a fear of being injured and alone are valuable to individuals and society at large.

\section{The Link between Gait and Health}

There are many methods for studying health in the aging. Over a half century ago S Katz et al. [6] suggested a framework referred to as the Index of Activities of Daily Living (ADL) which is still used today. The ADL focuses on a person's ability to achieve the basic functions of daily life such as eating, bathing, and using the restroom. The ADL is the basis of a number of ongoing research projects both at OHSU and PSU [7]. Although some of the elements of the ADL are able to be tracked in an automated environment, the tests were designed to be evaluated by caregivers some 50 years ago. By monitoring gait in the home it is possible to collect some metrics relevant to ADL such as walking speed and ambulatory time regularly and with much higher frequency than afforded by the typical annual assessment [8]. 
For a gait analysis system to be effective it must be able to detect clinically relevant changes in gait metrics. In the application of tracking recovery progress in stroke victims, Tilson et al. [9] study a group of 283 subjects with first-time stroke. A comfortable gait speed (CGS) change of $16 \mathrm{~cm} / \mathrm{s}$ was considered a meaningful improvement. The study tracked the progression of stroke sufferers at 20 days and 60 days post-stroke. Mean CGS changes of $18 \mathrm{~cm} / \mathrm{s}$ at 20 days and $39 \mathrm{~cm} / \mathrm{s}$ at 60 days poststroke were found. A CGS change of $16 \mathrm{~cm} / \mathrm{s}$ was found to have a sensitivity of $73.9 \%$ and a specificity of $57 \%$ for detecting improvement in subject's health.

In a study of Parkinson's disease (PD) medications Morris et al. [10] tested 15 subjects with PD for changes in gait metrics between peak dose time (.5 hours following dosing) and a half hour before re-dosing. At peak dose, PD sufferers had a mean walking speed of $103.2 \mathrm{~cm} / \mathrm{s}$ (standard deviation (SD) of 17.3), and a mean stride length of $108.5 \mathrm{~cm}$ (SD of 18.4). In the second test, walking speed was mean $78.6 \mathrm{~cm} / \mathrm{s}$ (SD of $28.7)$, and a mean stride length of $87.8 \mathrm{~cm}$ ( $\mathrm{SD}$ of 25.8$)$.

Schnall et al. [11] report on the gait characteristics of a soldier $(\mathrm{N}=1)$ with a traumatic hip disarticulation (leg loss, replaced by prosthesis). Between 3 and 38 months post-surgery the subject experienced step length changes of $12 \mathrm{~cm}$ on the prosthesis and $20 \mathrm{~cm}$ on the intact limb. The subject also experienced a change in walking speed of $29 \mathrm{~cm} / \mathrm{s}$, single support time $2 \%$ (prosthesis) and $0 \%$ (intact limb).

A study by Wet et al. [12] examines the contributions of abnormalities of gait biomechanics and gait characteristics to the energy cost of walking in older adults. The 
study of 50 elderly adults with mean age 76.7 years, $65 \%$ female composition, and an average of 4.3 comorbidities resulted in the following measures of gait characteristics: walking speed $88 \mathrm{~cm} / \mathrm{s}(84-9395 \%$ confidence interval (CI)) and stride time $740 \mathrm{~ms}$ (720$77095 \% \mathrm{CI})$.

The effect of therapeutic exercise on gait speed was studied by Lopopolo et al. [13] in a meta-analysis of 1,302 community-dwelling elderly people. The study found that Strength training and aerobic-plus-other-exercise training had statistically significant effects on habitual gait speed. Papers included in the meta-analysis report changes in gait speed pre-test to post-test of 0 to $7 \mathrm{~cm} / \mathrm{s}$ with one outlier of $17 \mathrm{~cm} / \mathrm{s}$ and the majority of the resulting changes in the $1-3 \mathrm{~cm} / \mathrm{s}$ range for both control and experimental groups. Overall, exercise training resulted in a habitual gait speed change of $1 \mathrm{~cm} / \mathrm{s}$.

The test-retest reliability of temporal and spatial gait characteristics was found to be good in a study using the GAITRite walkway system conducted by van Uden et al. [14]. The study measured the reliability of gait metrics including walking speed, step length, stride length, step time, stride time, single support time, and dual support time. The results can be seen in Table 1 .

The results of the research by Tilson et al, Morris et al., and Schnall et al. point towards potential use cases for in-home gait analysis. In each of these studies a temporal trend in gait metrics is used to find underlying patterns in the health of subjects. With an adequately precise system these tests could be completed in a smart-home environment instead of in a clinical setting. 


\section{Current Methods of Gait Analysis}

The current state-of-the-art for gait analysis methods both in the home and in clinical settings is described well in a survey paper Gait Analysis Methods [15] by Muro-de-La-Herran et al. In their paper the authors present a comprehensive survey of current gait analysis methods in the 2012-2013 time frame. The survey covers methods used both in a home and clinical setting. [15] begins with an introduction of key gait parameters such as is presented in this thesis. The authors provide some historical perspective on the progress of gait analysis methods, calling those which depend on a clinician's timing and judgement as being "semi-subjective." [15] argues that these methods are neither as accurate nor as precise as what they label "objective" methods. A more fitting duality might be to call the later automated and the former manual due to the distinction being made based upon the clinician being part of the measuring device.

In their survey, Muro-de-la-Herran et al. find that the current state-of-the-art of gait monitoring is two-pronged. The most accurate and reproducible systems are

\begin{tabular}{|l|l|l|}
\hline \multirow{2}{*}{ Gait Variables } & Mean Change Week 1 & \multicolumn{1}{|c|}{ to Week 2 $(95 \% \mathrm{Cl})$} \\
\cline { 2 - 3 } & Preferred Walking Speed & Fast Walking Speed \\
\hline Walking Speed $(\mathrm{cm} / \mathrm{s})$ & $4.65(1.27-8.03)$ & $2.96(-0.47-6.39)$ \\
Step Length $(\mathrm{cm})$ & $1.57(0.68-2.46)$ & $0.89(0.13-1.66)$ \\
Stride Length $(\mathrm{cm})$ & $3.17(1.36-4.98)$ & $2.11(0.49-3.74)$ \\
Step Time $(\mathrm{s})$ & $0.01(0.01-0.002)$ & $0.01(0.01-0.002)$ \\
Stride Time $(\mathrm{s})$ & $0.01(0.02-0.003)$ & $0.01(0.02-0.001)$ \\
\hline Single Support Time $(\mathrm{s})$ & $0.01(0.01-0.002)$ & $0.01(0.01-0.001)$ \\
Dual Support Time $(\mathrm{s})$ & $<0.001(0.01-0.01)$ & $<0.001(0.01-0.004)$ \\
\hline
\end{tabular}

Table 1: A Review of changes in gait metrics over time using the GAITRite walkway system. 
automated non-wearable systems used in clinical environments. The most usable in daily gait acquisition are automated wearable systems which the authors suggest as the ideal implementation for in-home use. The authors go on to offer guidelines for future research in new gait analysis techniques.

Automated methods include any system in which a computer and a set of sensors measure the gait characteristics of the subject in place of a clinician directly timing or otherwise measuring a gait metric. The authors delineate the automated methods into three categories: image processing, floor sensors, and sensors placed on the body. Image processing includes what are referred to as "structured light" methods including laser range finding. More traditional image processing techniques such as Kinect based methods are also grouped under image processing. In this nomenclature the IR/ RF sensor system presented in this thesis would at least partially fall under the heading of structured light methods. The authors go on to further delineation by grouping the image processing and floor sensors into the group non wearable sensors (NWS) and the body-worn sensors into the group wearable sensors (WS).

The authors describe the methods for image processing beginning with image thresholding to create a binary image with the figure made distinct from the ground. More time is spent analyzing various methods of depth measurement including time of flight (TOF) based systems, stereoscopic (multi-camera) systems, and laser scanning (LIDAR). The Microsoft Kinect sensor system is mentioned by name under the structured light heading because it projects an IR grid and uses an IR capture device to estimate depth measurement. For further detail on Kinect based methods this thesis has a 
detailed study of that system in Chapter 1 as well as another structured light system by ORCA tech called the passive infrared system (PIR).

Next Muro-de-la-Herran et al. present methods of gait analysis based on floor sensors, force plates and pressure sensors. The authors distinguish between the methods by pointing out that force plates measure pressure with 6 degrees of freedom, measuring vertical, horizontal, and rotational displacement along 3 axis. In contrast pressure sensors are simply a device for measuring the pressure caused when the sensor is compressed between a body part and the floor. Force plate and pressure sensor systems include both wearable and non-wearable systems. An example of a wearable system would be a sneaker insole which consists of a bottom layer of gel and a top layer of a piezoelectric material which is deformed in a measurable way as the subject walks. An example of a non-wearable system is the GAITRite Walkway which is described in detail in this thesis in Chapter 1.

Finally the authors present the inertial measurement unit (IMU) based gait analysis methods. These methods include one or more IMUs and gyroscopes which are mounted to a subjects clothing, footwear, or body to measure changes in moments of inertia of that body part. By knowing what part of the body an IMU is attached to, and by using multiple IMUs, a model of walking, joint flexion, and other biometrics can be constructed. An excellent example of one such IMU-based device is the Opal System by APDM, a Portland based company. Further detail on the Opal System can be found in Chapter 1. 
The survey goes on to compare prices, efficacy, and use cases among the gait analysis methods. Accuracy values are taken directly from publications on each sensor and as such are not in a single format, making it hard to compare them in a table. Pricing for each sensor is also vague, most price fields ranging by greater than an order of magnitude. The most interesting element of the analysis tables are the advantage/disadvantage columns which list the pros and cons of each method. At the broadest level, wearable systems are compared to non-wearable systems and the authors come to contrasting opinions to the ones in this thesis. Namely, the authors suggest that wearable systems are the primary systems appropriate for in home gait analysis and that their primary disadvantages are twofold: first is the complexity of algorithms needed to analyze IMU data, and second is the issue of battery life. In contrast this thesis presents wearable sensors as primarily a clinical technique because to effectively collect data sensors must be properly attached to the subject and monitored for functionality by clinicians.

For clinical purposes the authors of the survey suggest that non-wearable systems such as force plates and the GAITRite mat are ideal for data collections in a clinical setting because they are more accurate and provide more reproducible results. The authors point out that these systems tend to be very expensive and that it is impossible to monitor gait with them outside of the instrumented environment. Both these points are valid for the systems mentioned in this paper. The limitation of NWS to clinical settings is due largely to the fact that the systems listed are expensive, must be operated by clinicians, and are not able to distinguish among subjects. The authors point out that a 
key criteria to analyzing the efficacy of any system, WS or NWS, is to keep in mind the target user group and consider their specific needs regarding frequency of data collection, important gait metrics, and the effect of testing in a clinic versus in a home.

Finally the authors provide some guidance for future gait research, stating that in the future it is important that research focuses on sensors which can monitor gait daily while interfering the least with a subject's daily activities. Specifically the authors suggest that future gait research should focus on four different areas: (1) new sensors for gait parameter analysis, (2) power consumption, (3) miniaturization, and (4) signal processing algorithms. The authors deviate from the thinking of this thesis by stating that these objectives are best met by wearable systems such as the Opal device by APDM. It is the stated opinion of this thesis that a non-wearable system which is non-invasive, inexpensive, and accurate would be the ideal method for in home gait analysis because it would avoid the issues of body-worn sensing devices.

The following systems have been studied heavily and some are considered the gold standard devices (specifically the Vicon and APDM's Opal) for various types of mobility monitoring. These devices tend to deliver very high precision analysis of gait metrics. However, each of them has a reason for not being ideal for in-home gait monitoring. 


\section{The Vicon Camera System}

The Vicon camera system is the gold standard for gait analysis in clinical settings. Most techniques in the last 30 years have been compared directly to the Vicon system as a gauge of their accuracy. The Vicon camera system consists of one or more cameras positioned around a room to capture the motion of a subject. The subject wears two or more reflective dots on her person which the Vicon system detects through image analysis. The motion of these dots can be used to motivate a model of human physiology, thereby allowing for the reconstruction of joint angles, body segment positions and more.

These measurements can in turn be used for the analysis of physiological characteristics such as gait. In the field of gait analysis the Vicon system has been used for fall prediction, walking speed estimation, and other forms of gait analysis [16][17][18]. The Vicon camera system has been shown to be highly capable at distinguishing subjects with a history of falling from those without, using a dual-task test in which the subject carries water while walking [17]. One research group [16] was able to show that when used in a clinical environment with no occlusion the Vicon system is able to achieve an accuracy of better than 70 micrometers when measuring motion. The researchers are quick to note that this level of accuracy is highly dependent on a wellcalibrated system operating in a clutter-free environment.

Occlusion due to clutter is the Achilles heel of the Vicon camera system; because it relies on video analysis, any subject or part of a subject obscured from the Vicon's cameras is not available for analysis. This makes its use in an in-home setting require it to be carefully staged such that multiple cameras ensure no subject occlusion. In addition, 
the Vicon system is very expensive and therefore not likely to be installed permanently in a residence.

\section{Radio Frequency Antennae Array}

A recent paper by Adib et al. presents a very interesting result regarding clinical gait analysis via region level tracking [19]. While this work is not directly relevant to gait analysis it should be included because it has definite potential for in-home region level tracking and gait metric extraction. Physically the device is an array of RF antennae $2 \mathrm{~m}$ tall and $1 \mathrm{~m}$ wide. The entire device appears to be custom manufactured and consists largely of PCB antennae, for this reason it seems that the device could be fairly inexpensive to produce, although the author does not mention this in his paper.

Researchers showed that the RF array could be used to measure multiple subjects with decimeter accuracy, even when obscured by walls. In addition the device can be used to measure breathing and gestures of each subject, leading to the supposition that it could also be used for gait analysis in the future. For the purposes of the initial paper, the system was tested in a research environment as well as a simulated office.

One finding of this research is that the system suffers from obfuscation as it tries to track multiple subjects: those nearer to the system block those further away. To solve this problem the researchers use a data association technique which they call successive silhouette cancelation in which the nearer object is tracked and then subtracted from the 
data. These steps are repeated until all objects are tracked. Later it will become apparent that the IR gait system presented herein uses a very similar technique for data association.

Results from this the initial testing of this system are highly encouraging and suggest that very detailed region level tracking could be inexpensively achieved in the future. Concretely, the system was able to localize up to 5 users with a median accuracy of $8-18 \mathrm{~cm}$ in the $\mathrm{x}$ direction ( orthogonal to the array plane) and $7-11 \mathrm{~cm}$ in the $\mathrm{y}$ direction (parallel to the array plane). The discrepancy in the accuracies in the $\mathrm{x}$ and $\mathrm{y}$ directions is due to the shape of the antennae array which is more sensitive to time-of-flight errors in the $\mathrm{x}$ direction than in the $\mathrm{y}$ direction. The system can also detect pointing gestures in three dimensions with 8-16 degrees of accuracy.

\section{The Opal System by APDM}

The Opal system manufactured by APDM is a body worn device or set of devices each of which consist of an accelerometer a magnetometer and a gyroscope as well as data storage and communications hardware. Devices can be used in configurations of up to 24 Opal devices. Opal devices can come pre-attached to an object, such as a person's shoes, but is more commonly positioned by a clinician for an in-clinic task [20].

The Opal system is highly accurate due to its ability to integrate multiple devices and multiple sensors in each device. In so doing it is possible to measure a wide variety of gait, balance and postural metrics. The device has been tested both in clinical and 
home settings [21] for gait analysis as well as a wide range of other uses including physiological monitoring of athletes and soldiers after a concussion event [22].

The sole deficiency of the Opal design is that if the device is not being worn, it will not monitor a person's motion. Because many persons with gait impairment also suffer from cognitive impairment, there is a serious difficulty with asking those persons to remember to wear or carry a device.

\section{The Kinect by Microsoft}

The Kinect was a device designed by Microsoft originally for gaming with their dedicated game platform, the X-Box. Later the system was released as a Windows OS device which could be used along with a software development kit (SDK). The Device itself consists of an IR depth camera, RGB camera, and an array of microphones, all mounted on a motor-driven pivot.

Body position can be tracked using the IR depth camera and the SDK which outputs a skeletal model of a person within $4 \mathrm{~m}$ of the camera. The Kinect can be used to monitor joint position and angle, spatiotemporal gait characteristics, and other physical motion of the body [23][24][25]. The Kinect is similar to the Vicon camera system in that it relies on line of sight imaging to capture body motion. Therefore it suffers from much the same deficiencies as does the Vicon system such as varying brightness in a room caused by windows, obscuring of the subject due to clutter, and the shape of the room being unconducive to the sensors range limitations. 
Researchers found it especially difficult to directly track as subject's legs when not in a clinical environment due to the common low-lying clutter of a home such as chairs, tables, couches, lamps, etc. Stone et al [25] suggests a solution for the issue of lower-limb detection. Using a Gaussian Mixture Model (GMM) of features including walking speed, subject height, and stride length the researchers could estimate a gait cycle by measuring fitting the features of a subject to the GMM. The estimates from this technique were not found to be highly accurate.

Because of the difficulties of in-home use, as well as the reticence of elderly people to have a video camera in their homes, the Kinect is best suited as a low-cost, highly accurate clinical alternative to the Vicon camera system.

\section{The Benefits of In-Home Gait Analysis}

Collecting data continuously at home it is possible to have a far richer data set than by doing the same in a clinical environment. The switch to in-home testing is a new one. 15 years ago the ability to place inexpensive sensors in a person's home and to monitor those sensors remotely did not exist. Today, while the technology exists, it is new and therefore important that good implementations are found in the current framework of in-home healthcare systems. From previous research, there are known tests for gait as a proxy for cognitive decline. The simplest such test is walking speed. To test walking speed a subject is asked to walk a predefined route, often a hallway or marked path as quickly as possible without running. The subject's time is measured using either 
an automated triggering system such as photovoltaic sensors, or a tester manually timing the walk between two points. A number of studies point to the use of velocity as a predictor of cognitive decline both in healthy and already diagnosed subjects [26] [27]. Gait characteristics other than walking speed are indicative of cognitive changes in older adults; Ble et al. points to stride length as an indicator of executive function [28], while Bowen et al. indicate a correlation between dual support time and the effects of a stroke [29]. Other gait characteristics include step length, step width, step/extremity ratio and stride width [30]. All of the metrics listed here are detectable using current systems such as the GAITRite gait mat. Many of the current devices are too expensive to be used outside of a clinical setting. Because of this limitation the results from these devices suffers from an inherent performance bias as when subjects are asked to come to a clinic and perform a task they know they are being measured and attempt to perform accordingly. Such metrics are only obtained at the frequency with which a subject can go to the clinic for testing. An affordable solution which could be deployed in a home would therefore have the opportunity to provide more consistent metrics with a higher degree of accuracy than its more expensive counterparts in the clinical setting.

\section{Current In-Home Gait Analysis Systems}

As a field of study, in-home device free gait detection is relatively new but the PSU BSP lab is not the first research group to address the issue. Other groups have provided more accurate systems, such as MIT's RF tracking system [18], more detailed systems, such as the GAITRite gait mat [14], or more simple systems such as 
ORCATECH's ceiling mounted IR array [8]. The following is a description of some of the other systems currently available which attempt to solve the same or similar problems.

\section{The GAITRite Walkway}

The GAITRite walkway (GR) is the current gold-standard for gait analysis systems both at OHSU and at PSU. Much of the reason for our use of the system is its simplicity and accuracy. With a single researcher the system can be transported, setup and operated with ease. Because the system is entirely self-contained in a flexible mat it is consistently accurate. The proprietary GR software allows for walks to be exported at various levels of processing for use in analysis and comparison.

The system consists of a portable walkway constructed of pressure sensors sandwiched between a foam layer beneath and a nylon layer above. The system connects to proprietary software on a Windows PC. The software detects and analyzes the timing and placement of footfalls, recording individual footstep data for analysis and replay. The active sensing portion of the mat measures an active area of $61 \mathrm{~cm}$ by $732 \mathrm{~cm}$ and consists of nearly 28,000 individual sensors placed $1.27 \mathrm{~cm}$ apart each sampling at $80 \mathrm{~Hz}$. Testing suggests that individual step values were within $1.5 \mathrm{~cm}$ and 0.02 seconds $80-94 \%$ of the time. Values recorded by the GAITRite and a second system used for benchmarking did not vary from one-another significantly in one such test. 
Because of its cost, size, and bulk (noted by other researchers as a potential tripping hazard [31]) the system is not suitable for unattended use. Because it must be operated by a researcher for each walk it would be impossible in its current state to integrate into a smart-home environment. In addition it has no ability to separate individuals and therefore could not be functional in a multi-user home.

\section{ORCA Tech PIR system}

The ORCATECH passive infrared system (PIR) is a ceiling mounted array of passive infrared sensors meant to detect motion occurring directly beneath them [32][33]. These sensors are relatively easy to mount and provide their data wirelessly to a central database. In my experience setup has been a challenge with these sensors due to a toobroad IR aperture which makes their motion detection imprecise and a difficulty in getting the software setup correctly. Despite these difficulties the PIR system is unobtrusive, relatively inexpensive, and provides walking speed data on subjects walking below it in a hallway.

A row of 4 sensors is placed in a line overhead in a hallway or other place where people will walk in a straight line. Each PIR sensor has its field of view restricted by a taped aperture which allows the sensor to have a $+/-6 \mathrm{~cm}$ accuracy at a distance of $90 \mathrm{~cm}$. Due to inaccuracy in the application of the tape aperture, some form of ground truth must be used to calibrate the system upon setup. For the purposes of testing the GAITRite 
system is used for calibration. By measuring the differences in timing and the known distance between sensors the walking speed can be found.

Due to the reduced view of the sensors with taped apertures, some sensors failed to fire when a subject walked past them. When using the GR and the PIR sensors the walking speed correlated to ground truth results with between 93\% accuracy with 2 sensors firing and $99 \%$ accuracy with 5 sensors or more firing. The mean walking speed of subjects in this segment of the testing was $139.5+/-26 \mathrm{~cm} / \mathrm{s}$. In-home testing provided an additional 100,000 walking speed samples over three months across 18 homes. Mean walking speed across subjects was $99.3+/-9.2 \mathrm{~cm} / \mathrm{s}$.

Physical measurement of in-home sensor lines is not precise, and therefore walking speeds are relative. The sensor system can collect an amazing amount of data because it is in constant use. This is one big benefit of an at-home system over its clinical counterparts. Unfortunately the sensor system only measures relative walking speed, requires calibration, and does not have any other pertinent gait metrics.

\section{The IR Distance Sensor System}

After building the first prototype of our IR gait detection system one of our primary investigators, Dr. Peter Jacobs from the OHSU Biomedical Engineering Dept. brought to light a paper on an extremely similar system from a research group in Taiwan. This paper titled Easy-to-install system for daily walking ability assessment using a 
distance sensor array by R Fukui et al. [31] describes a system which is uncanny in its similarity to our own.

The system is described as an easily installed gait assessment system designed to measure walking speed and step length. The system does not require any initial configuration or 'learning' because it relies entirely on a heuristic approach for data analysis.

The system is constructed as a 1.2 meter strip with 24 IR sensors (Sharp GP2Y0A21YK0F), the same sensor we used for the subject ID system which we began research on in early 2014 and initially submitted for publication in February of 2015. The sensors are grouped onto custom PCB boards in groups of 4 which allow the system to be expanded or repaired. The IR transceivers run at $20 \mathrm{~Hz}$ and use triangulation to measure distance. They are built into a rigid platform $6 \mathrm{~cm}$ high requiring a ramp to use. The sensors claim to be able to measure from 10 to $80 \mathrm{~cm}$ with a resolution of $60 \mathrm{~Hz}$, although our independent testing of the sensors suggests that possibly their range is closer to half that. The modular nature of the system allows it to be easily repaired but requires a fixed distance between sensors. At a length of 1.2 meters the sensor strip can catch one $80 \mathrm{~cm}$ walking step only if it is well centered in the walking window. An ATMega microcontroller acquires data from the sensor array via 216 channel multiplexers. The system is powered by a wall wart power cord and transmits data wirelessly using an XBee wireless transmitter. 
The overall flow of the data processing is as follows: data is acquired using the IR gait array. Next, regular walking data is extracted. This is done by thresholding the raw data at $800 \mathrm{~mm}$, which is the maximum range of the sensor. Any data acquired at less than $800 \mathrm{~mm}$ is therefore considered part of a walk. Walks are considered when they take place in less than 3 seconds (corresponding to $40 \mathrm{~cm} / \mathrm{sec}$, a very slow walking speed, even for the elderly). Next, step length is estimated by looking at the sum of energy ("reaction") across all time. Figure 1 depicts the "reaction" as a bar graph. By performing peak detection and measuring the distance between adjacent peaks (in figure 1, peaks Xc1 and $\mathrm{Xc} 2$ are considered adjacent), a step length estimate can me calculated as step length $=$ $\Delta p e a k *$ peak seperation. Walking speed is calculated as $\frac{\Delta \text { Dist }_{\text {Heel Strike }}}{\Delta \text { Time }_{\text {Heel Strike }}}$ where $\Delta$ Dist $_{\text {Heel strike }}$ is the distance between the heel strike of a foot and that of the opposite foot, and similarly $\Delta$ Time $_{\text {Heel strike }}$ is the timing difference of the two heel strikes. Heel strikes are determined by using the Hough Transform, which identifies straight lines in a binary 2D image, to identify when a foot is stationary. The onset of the stationary foot timing is then considered to be a heel strike. Finally, step duration is calculated as the period of time the Hough transform determines a foot to be on the ground.

Although Fukui et al. do not report algorithms or figures for single leg stand time or dual leg stand time, this could also be determined using the data from the Hough transform. The algorithms used in this section are highly dependent on data with a very high SNR. The benefit of the algorithms presented in this paper is that, given a high SNR, they are quite effective for analyzing some gait metrics. The algorithm is not at all robust to a low SNR. 
Walking step length ranges from $30-60 \mathrm{~cm}$, walking speed ranges from $.5-1 \mathrm{~m} / \mathrm{s}$. The proposed algorithm can estimate step length with an accuracy of $15 \mathrm{~mm}$, walking speed with an accuracy of $.05 \mathrm{~m} / \mathrm{s}$. These results were compared to ground truth acquired from a pressure mat which resulted in a sensitivity of $100 \%$ and a specificity of $97.5 \%$.

The algorithm used to analyze walks from the IR distance sensors does not measure single support time, step/extremity ratio, or stride width. It may be within the purview of this research group to do so but their current work does not show these results. In addition the system does not work with a cane and assumes that the person is walking without shuffling, both of which are sufficiently common in older adults to merit inclusion into such a system. While the study results are very impressive given the simplicity of their algorithm, the primary weakness is their lack of testing on subjects with non-standard gait characteristics such as walking with a cane or shuffling walks. Because the system does not require training certain assumptions are made such as the fact that the walker will not

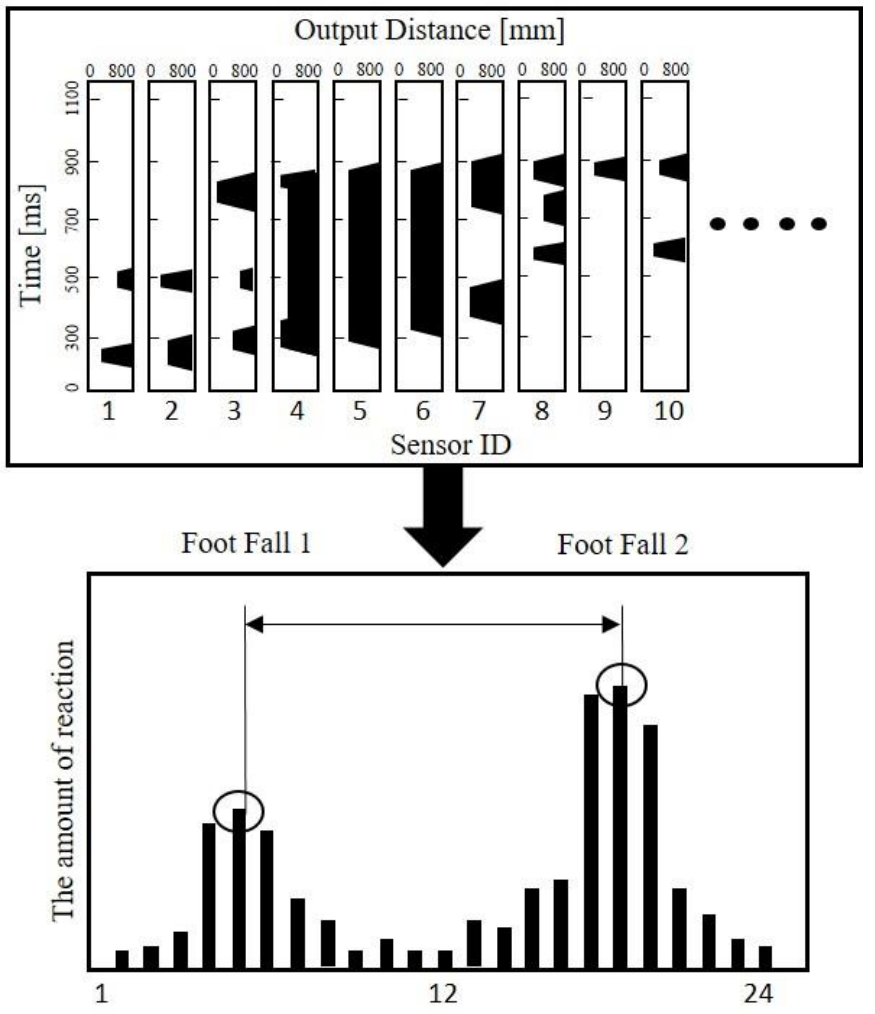

Figure 1: Reproduction of a figure from Fukui et al. The distance between peaks in the lower figure gives distance and the breadth of the plateau associated with sensor 5 in the top figure gives timing. 
shuffle while walking. In addition because the system disregards walks in which a single sensor does not fire it is possible for the entire walk to be missed if a subject lifts her foot higher than the sensor height of $5 \mathrm{~cm}$ (less than 2 inches). In addition the systems length does not allow it to measure step-to-step gait variability, an important measure of fallpotential. Finally, and most importantly, the system does not distinguish among multiple subjects, therefore relegating it to a single-subject dwelling.

Searches for the literature review and existing-systems overview conducted in this chapter were done using Google Scholar, Elsevier, and the PSU Library scientific periodicals search resource at library.pdx.edu. Search terms for these searches included: elderly, elderly care, aging-in-place, American demography, gait, gait analysis, activities of daily living, spatial, temporal, Shimmer gait device, GAITRite gait device, Opal gait device, Onspot gait device, Microsoft Kinect, IR gait detection, walking speed, gait detection, tagged, tag-free, in-home, and more. 


\section{Chapter 2: Design Methodology}

Design requirements for a gait analysis system are dictated by the context in which it is to be used. The system described here has the goal of replacing some clinical gait analysis with in-home gait analysis. In the previous section the work by Tilson et al. suggests that in monitoring meaningful changes in comfortable gait speed post-stroke a system should be able to detect changes of in walking speed of less than $16 \mathrm{~cm} / \mathrm{s}$. Morris et al.'s study on the efficacy of medication for Parkinson's disease a gait detection system which can distinguish walking speed with at least $12 \mathrm{~cm} / \mathrm{s}$ accuracy, and stride length with an accuracy of $10 \mathrm{~cm}$ would be useful for detecting a drop in medication levels, possibly due to an elderly person forgetting her medications. Schnall et al. suggests a walking speed accuracy of $14.5 \mathrm{~cm} / \mathrm{s}$ and step length accuracy of $6 \mathrm{~cm}$ would be sufficient to measure changes in gait characteristics for a person with traumatic hip disarticulation over the course of a 3 year study. Van Uden et al.'s research on test-retest reliability of temporal and spatial gait characteristics using the GAITRite walkway show that extremely fine-grained statistics could be useful for detecting changes in walking speed, step length, stride length, step time, stride time, single support time, and dual support

\begin{tabular}{|l|l|l|}
\hline & accuracy & \\
\cline { 2 - 4 } Gait Variables & Lowest & Highest \\
\hline Walking Speed $(\mathrm{cm} / \mathrm{s})$ & 16 & 1 \\
Step Length $(\mathrm{cm})$ & 10 & 0.1 \\
Stride Length $(\mathrm{cm})$ & 5 & 0.01 \\
Step Time $(\mathrm{ms})$ & 10 & 2 \\
Stride Time $(\mathrm{ms})$ & 10 & 3 \\
Single Support Time (ms & 10 & 1 \\
Dual Support Time (ms) & 20 & 2 \\
\hline
\end{tabular}

Table 2: Desired Accuracy for a Gait Analysis System. 'Lowest' column depicts lowest necessary accuracy, 'Highest' column depicts highest. 
times. Temporally, a system able to detect changes on the order of $10 \mathrm{~ms}$ and spatially on the order of $1 \mathrm{~cm}$ would be ideal for this type of study. By viewing these experiments a desirable range of gait metric sensitivity can be achieved. This result is visualized in table 2. The first column depicts a gross measure while the second column depicts a fine measure. A gait analysis system would ideally be as or more sensitive than the fine measure column, but should certainly be between the two to be useful in replacing clinical data collections with smart-home data collections.

The system discussed in this paper is the second prototype in an ongoing research project attempting to reliably and accurate track gait. As such, while table 2 represents final figures for accuracy of gait metrics which are desirable, there were other factors which were taken into account for the hardware development of the gait detection system. It should be noted that design decisions were made by the research group as a whole

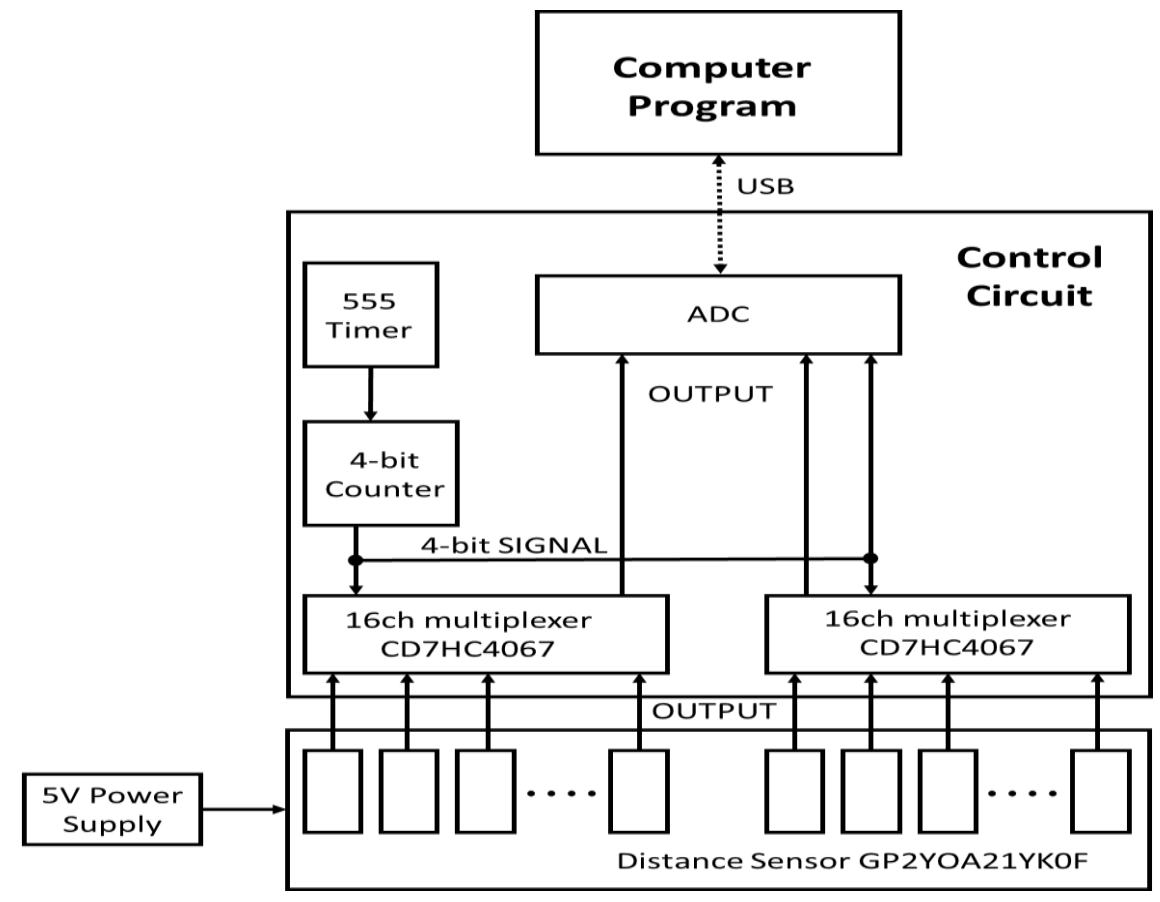

Figure 2: A block diagram of hardware design. 
under the leadership of Dr. Eric Wan. The final implementation of prototype 2 (referenced herein as the system) was done almost exclusively by Erich Schafermeyer with the exception of some soldering help by Shadman Samin and some case construction help from Tanisha Payne. Prototype 3 is being constructed entirely by Shadman Samin and Tanisha Payne. Prototype 1 was constructed in large part by Shadman Samin with some help from Erich Schafermeyer. All algorithms and software described herein were designed by Dr. Eric Wan and Erich Schafermeyer with the essential support of Walter Woods, Dr. James McNames, and Dr. Melanie Mitchell.

The system was tested following Institutional Review Board (IRB) protocols for the data collections. IRB oversight was provided by the OHSU IRB and was approved through the PSU IRB approval process.

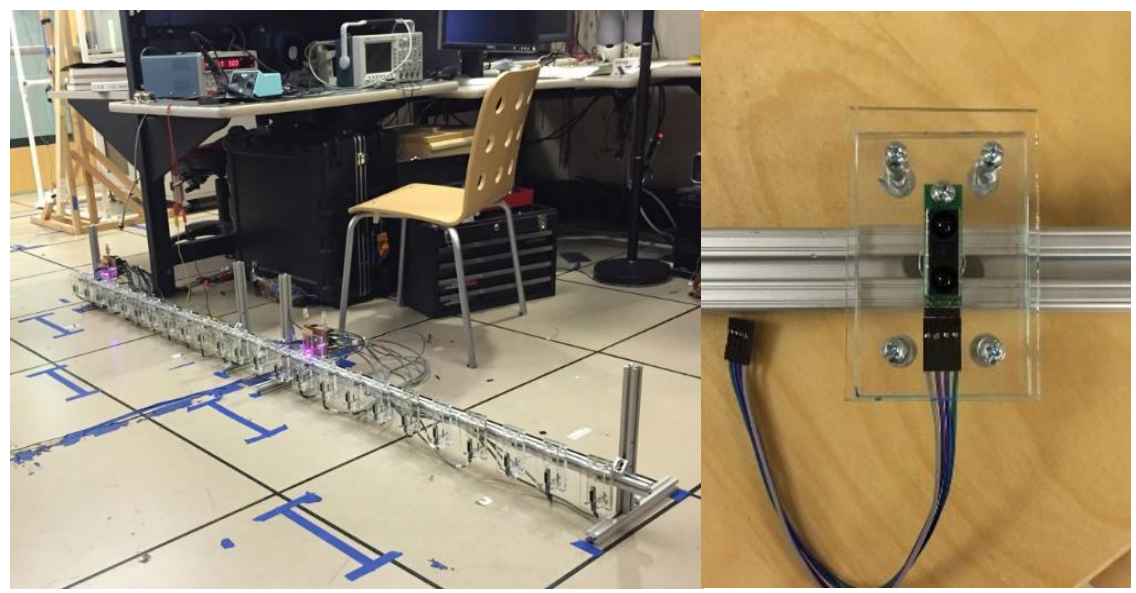

Figure 3: The second prototype of the IR sensor array. Left image depicts an array of 24 sensors in a simulated hallway setup in the BSP lab. Right is a detail showing the sensor in an acrylic housing attached to extruded aluminum rail. 


\section{Hardware}

The hardware design for the gait detection device was a critical decision in the design process. The design of the hardware determines how and where the system can be used, how the code base for the system will be developed, and how robust the system is to modification, repair, and testing. Deciding on the size and material for the mounting system, the layout for the electrical system, and choosing the sensors themselves were all important elements of the design process.

To effectively measure gait characteristics of a person walking down a hallway it is ideal to capture at least two full cycles of the subjects walk. Assuming a stride length of 1 meter, the array would, ideally, be at least 2 meters long. To be certain two strides are captured, the system was designed to consist of two rails, each $122 \mathrm{~cm}$ long, for a total length of 2.44 meters. Assuming an average walking speed of $1.4 \mathrm{~m} / \mathrm{s}$, and that a foot will spend roughly half its time stationary, a spacing for the sensors can be calculated such that a sensor will never be passed by the foot without taking a measurement:

$$
\frac{1.4 \text { meter }}{\text { second }} * 2 * \frac{1 \text { sample }}{60 \text { seconds }} \cong \frac{46.6 \mathrm{~mm}}{\text { sample }}
$$

Note that in the above calculation the multiplier (2) is used because half a foot's time is spent not moving, therefore it must move twice as fast as the torso to travel the same distance over the same time period. Thus the foot can move up to $47 \mathrm{~mm} / \mathrm{sample}$ and the sensors should be at least $50 \mathrm{~mm}$ apart to ensure that the foot does not pass a sensor without being sensed. 
Figure 2 shows a block diagram of the hardware system. In the diagram 24 channels of distance data are collected by 24 IR sensors which are powered in parallel by a 5v DC power supply. Sensor data is multiplexed and sent into an analog-to-digital converter (ADC). Data is then acquired by a computer program for de-multiplexing and analysis. The system is constructed as two identical rails, each $1.22 \mathrm{~m}$ long. 12 sensors are spaced along each rail at $101.6 \mathrm{~mm}$ intervals. A single control circuit (one MUX, clock, power supply) is mounted to each rail.

The choice to break down the system into 12 sensor sub-segments was largely a result of the ADC used. To have a sufficiently fast sample rate on the ADC while keeping costs low it was necessary to use an ADC with less than 25 channels input, which would be necessary if not multiplexing the sensor data before passing it through the ADC. To reduce the number of channels entering the ADC a MUX was chosen which could handle a large number of sensors in case it was ever decided to add more sub-segments to the system. 


\section{The Sensor}

The sensor chosen was an infrared (IR) light transceiver (Figure 3) (Sharp GP2Y0A60SZXF_E). Light is emitted from an IR light emitting diode (LED) and received by a position sensitive device (PSD). The distance from the IR sensor to the target object is measured by triangulation and then sent to the collection device as an analog signal in the $3.6 \mathrm{v}$ to $.6 \mathrm{v}$ range. The spec sheet for the Sharp IR sensor states that the sensor has a range of $10-80 \mathrm{~cm}$, although evidence would suggest that the range is closer to $20-60 \mathrm{~cm}$. The sensor housing includes an oscillator which samples the IR receiver at a rate of 60 times per second as well as a low pass filter (Figure 4). Empirical evidence suggests that the closest these sensors can be spaced without causing excessive interference with each other is around $10.2 \mathrm{~cm}(\sim 4 \mathrm{in})$ apart. This testing was done by Shadman Samin in the early phases of prototyping and again between prototypes 2 and 3 .

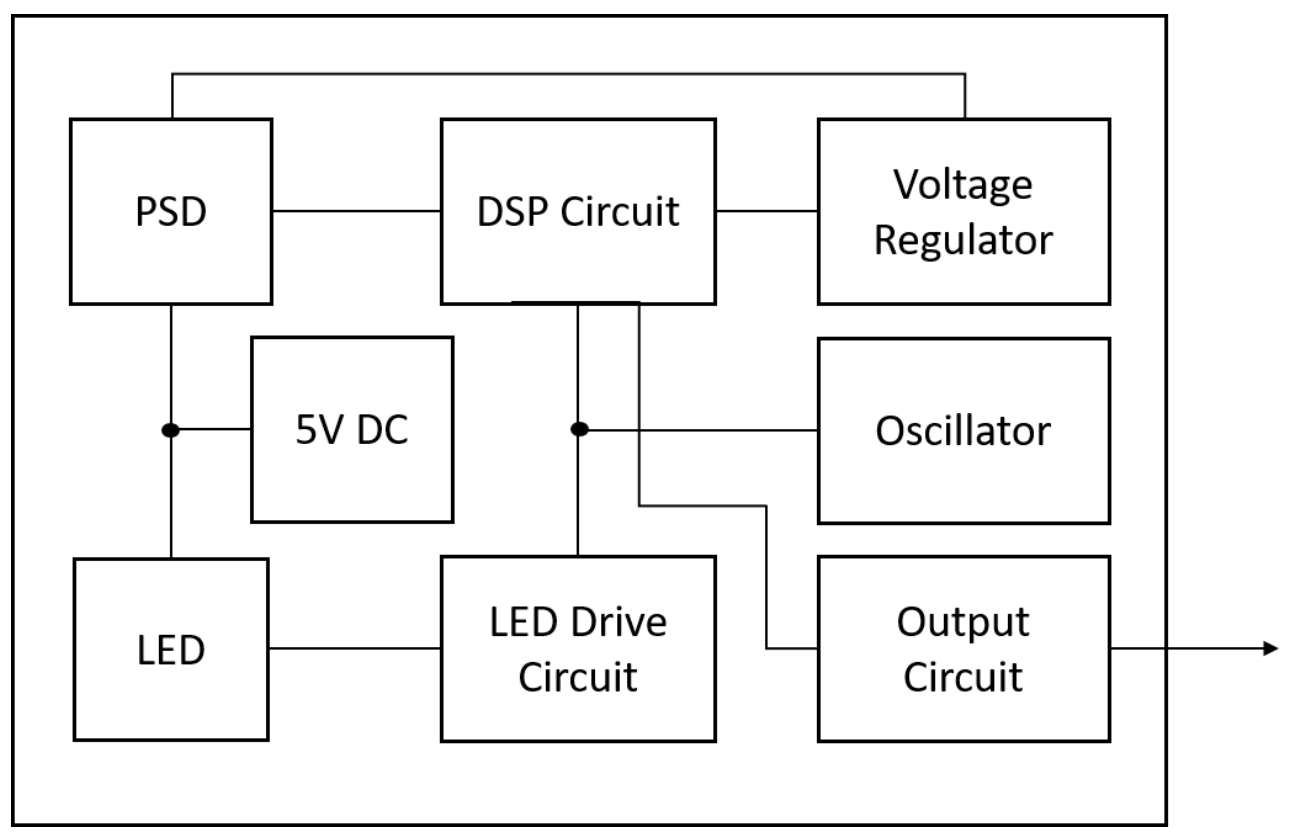

Figure 4: Reproduced from the SHARP GP2Y0A60SZXF_E infrared sensor specification sheet. This image depicts the sensor layout. 
Tests suggested that placing the sensors closer together than $10.2 \mathrm{~cm}$ resulted in noise increases in the captured IR signal of one or more sensors in the array. It would be ideal in a future prototype to space the sensors at a closer spacing so that a finer distance could be measured. By placing all sensors along one wall the system could eventually be installed in a hallway and powered by a single outlet. In addition, transmitter/receiver pairs would not measure distance, only a 'broken link' and therefore would provide less information that the sensors used here.

\section{The Mounting System}

A single hole in the sensor is the only point by which to mount the sensor to a rail. The sensor package contains both the transmitter and receiver thereby allowing the control circuit and sensor array to be mounted to a single wall (Figure 3). The benefit of mounting to a single wall is that in a hallway with doors or objects along one side the sensor system would still be able to be used. In addition the sensor system need only be powered by a single outlet instead of by one outlet for the transmitter array and a second for the receiver array on the opposite side of the hallway. A rail was constructed in two $4 \mathrm{ft}$ segments, each on a pair of legs which could be adjusted in height. The rail itself is made of aluminum

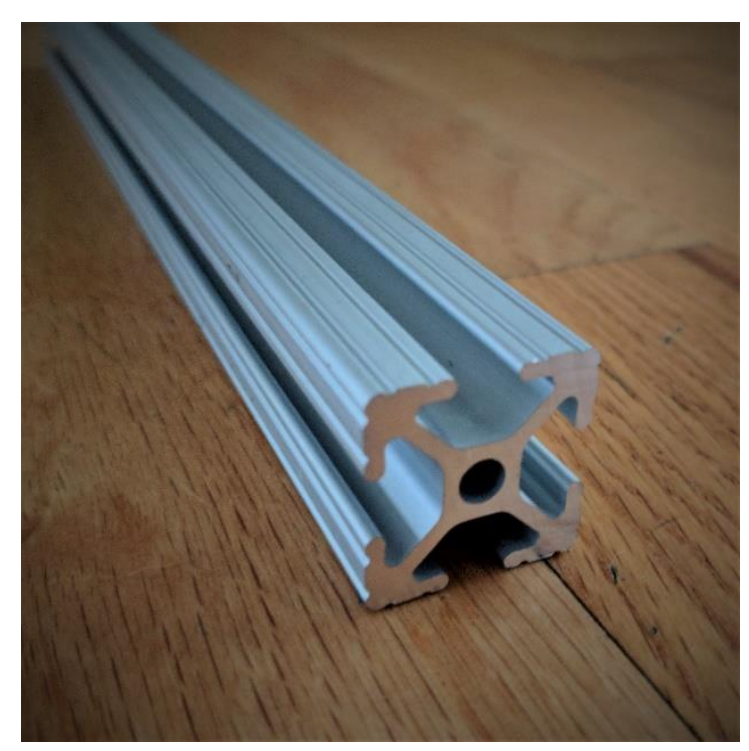

Figure 5: 80/20 1x1 inch extruded aluminum railing. 
extrusion called 80/20. The extrusion rail system consists of modular extruded aluminum which can be cut and bolted to itself easily (Figure 5). The continuous channel in the rail allows the height and placement of sensors to be infinitely adjustable. To connect the sensor to the rail a box is constructed of three Plexiglas sheets as well as bolts and nylon washers. The sensor itself is mounted to the front panel of Plexiglas which is then mounted to the rear panels by a bolt at each of four corners (Figure 3 ). The two plates are held off of each other by nylon spacers to which can be added nylon washers to adjust the length of the spacer. By adjusting the length of the spacer the vertical and horizontal angles of the front plate and therefore the IR sensor can be adjusted to angle the sensor. This is an essential degree of freedom because the sensors, rail, and cases are not precisely constructed. If the sensors were not directly adjustable the ability to ensure their orientation would be greatly reduced.

\section{The Control Circuit}

The control circuit is designed around two multiplexers. Each multiplexer takes in 16 channels and outputs 1 channel at 16 times the rate. The IR sensors switch at $60 \mathrm{~Hz}$ so the multiplexer must sample each of the 16 channels in $1 / 60^{\text {th }}$ of a second. In that same time all 16 inputs sampled are concatenated and sent out as a single channel. The multiplexer is driven by a 4 bit counter. Each count corresponds to one of the multiplexers input channels. The input is then drawn from the corresponding channel as the 4 bit counter switches. In this application the 4 bit counter is driven in turn by a 555 timer running at $8 \mathrm{kHz}$. Which allows each sensor to be sampled 8 times per $60 \mathrm{~Hz}$ cycle. 


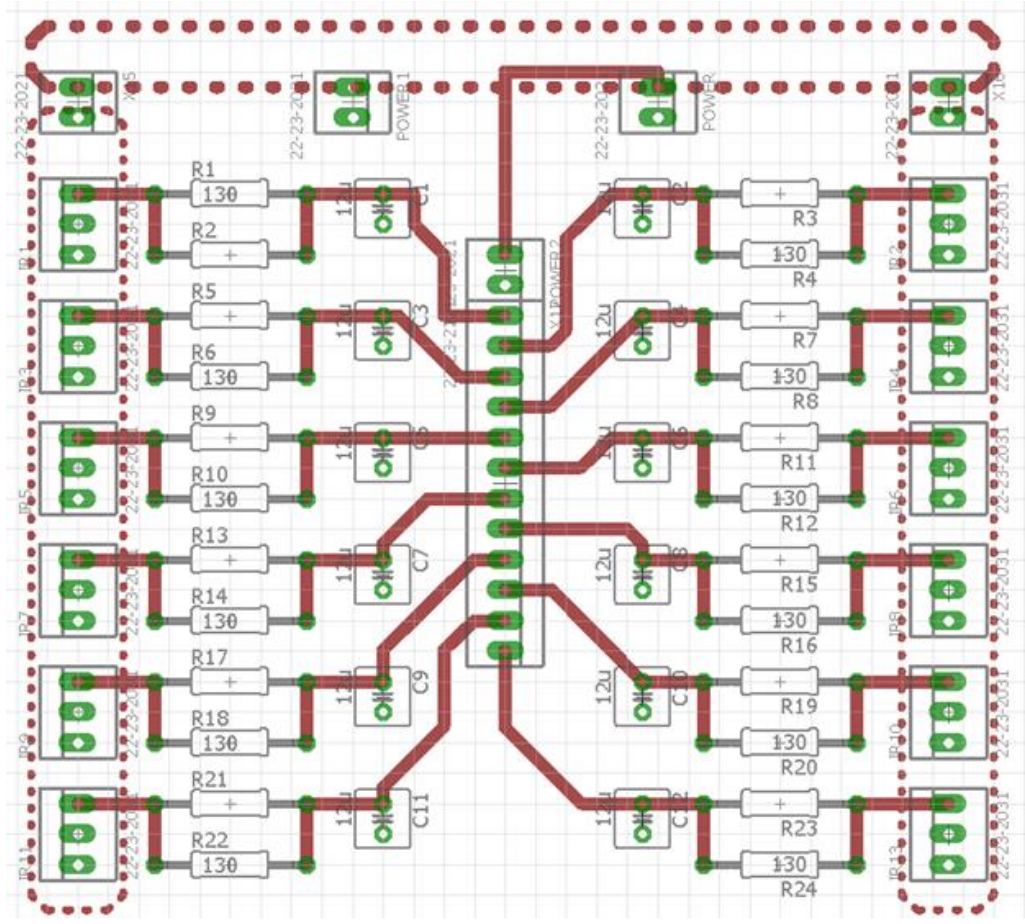

Figure 6: Control Circuit Board.12 IR sensors attachment points with shunt circuits entering a single line female pin set where a daughter board is attached carrying a MUX.

It is important to sample the sensors multiple times per $60 \mathrm{~Hz}$ cycle and find the average of multiple values to account for noise in individual samples. Because there are 24 sensors, only 12 channels of each multiplexer are used. The outputs of the MUX as well as that of the 4bit counters are taken as inputs to an analog to digital converter (ADC) which samples at a rate of $64 \mathrm{kHz}$, allowing it to capture the MUX signals with 8 samples per switch as well, again to account for noise in switching. A Scientific Instruments ADC was used which is capable of sampling 8 channels at up to $250 \mathrm{kHz}$. Power is provided to the sensor system by a dedicated DC power supply running at $5 \mathrm{v} 1 \mathrm{~A}$. The control circuit was implemented on a custom routed PCB which was designed using EagleCAD with the help of John Folsom at MotioSens and cut on the LID PCB router (Figure 6). In the board schematic mounting points can be seen for a shunt circuit which is a capacitor bridging 

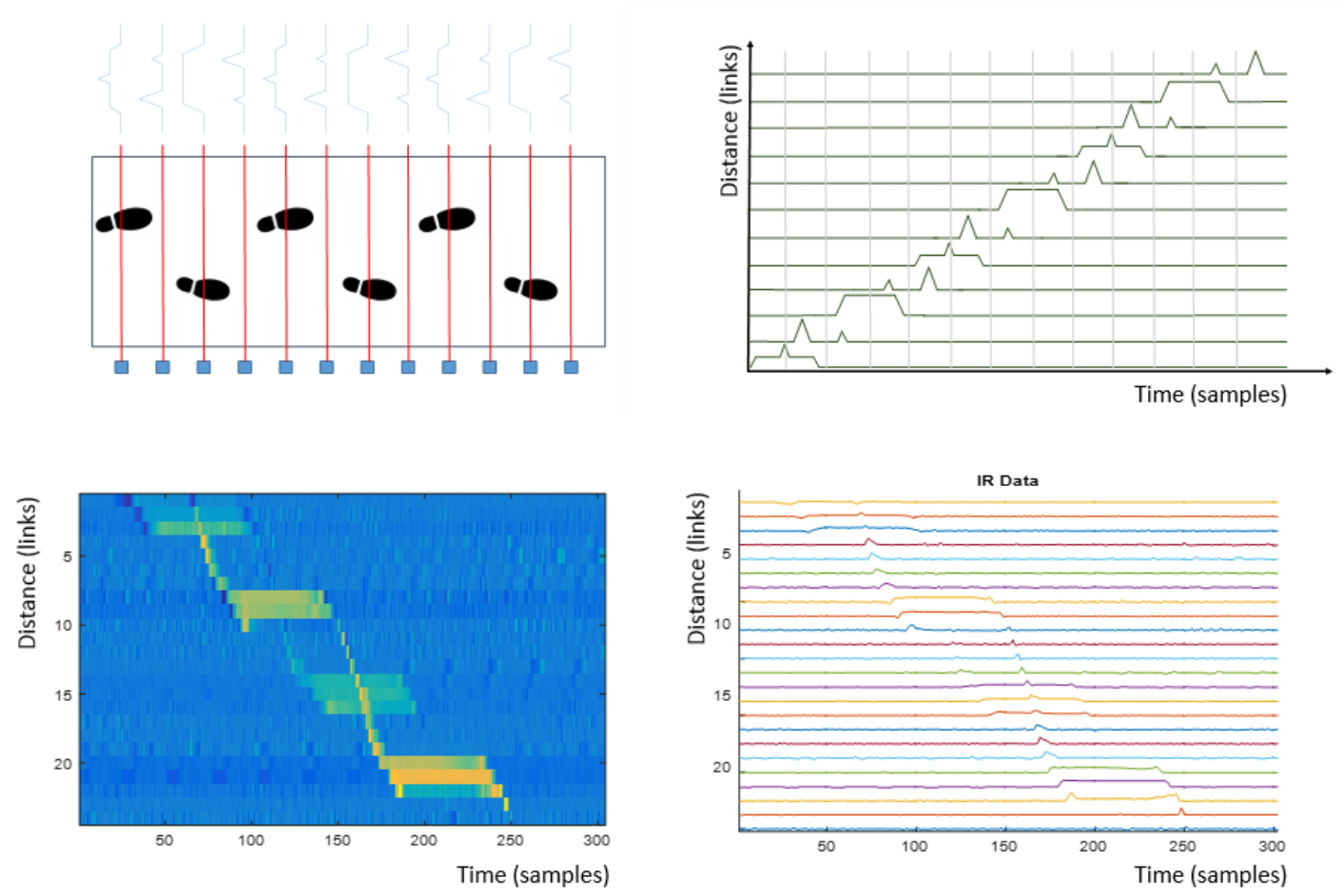

Figure 7: An intuitive look at the waveforms resulting from a walk across 12 sensors (half the number actually being used). The figure to the top left associates' step patterns with waveforms while the figure to the top right shows the resulting walk across all links. Lower figures show real data as heat map (left) and traces (right).

the power and ground line for each sensor. The shunt circuit in general has many uses as a circuit breaker, reverse polarity protection, or low pass filter. In this case it is being used to redirect high-frequency noise to ground. A second low-pass filter circuit is also integrated into the control circuit design. All holes in the design are through-plated allowing the bottom of the board to be soldered to more effectively. A final consideration are the wires connecting the sensors to the control circuit board. These wires can be as much as $140 \mathrm{~cm}$ long and act as excellent antennae for receiving electromagnetic noise from the surrounding environment. Because wall power in the US runs at a $60 \mathrm{~Hz}$ cycle and the IR sensors are sampling at $60 \mathrm{~Hz}$ it could be extremely difficult to remove any noise (called mains hum in audio applications) introduced by wall power. It was therefore 
important that the wires were well shielded to avoid the introduction of noise from other electrical devices in the room.

\section{Ideal Data Example}

Ideal data coming from the hardware system is depicted in Figure 7. In the figure a walking space is depicted as a rectangle outlined in black. The rectangle is transected by red lines which depict the IR beams emanating from the sensors which are depicted as small blue squares. On the mat can be seen footprints which depict the positions at which a subject places her feet while walking. The foot nearer to the blue sensor boxes is referred to as the near foot while the foot further away is referred to as the far foot. Above the walking area can be seen an idealized version of the waveform which will be acquired at that link based upon the subject's foot positions. There are four basic waveforms depicted. At the first link from the bottom a waveform with a broad, low plateau bisected by a spike is visible. This waveform is created by a far foot stationed in the link, creating the plateau, and a near foot swinging past, creating the spike. At the second link from the bottom a waveform with a tall spike and a short spike is visible. This waveform is created by a near foot swinging followed by a far foot swinging. At the third link from the bottom a waveform with a broad, high plateau is visible. This waveform is created by the near foot stationed in the link. Because the far foot is obscured by the near foot, the far foot does not influence the signal at this link. At the 


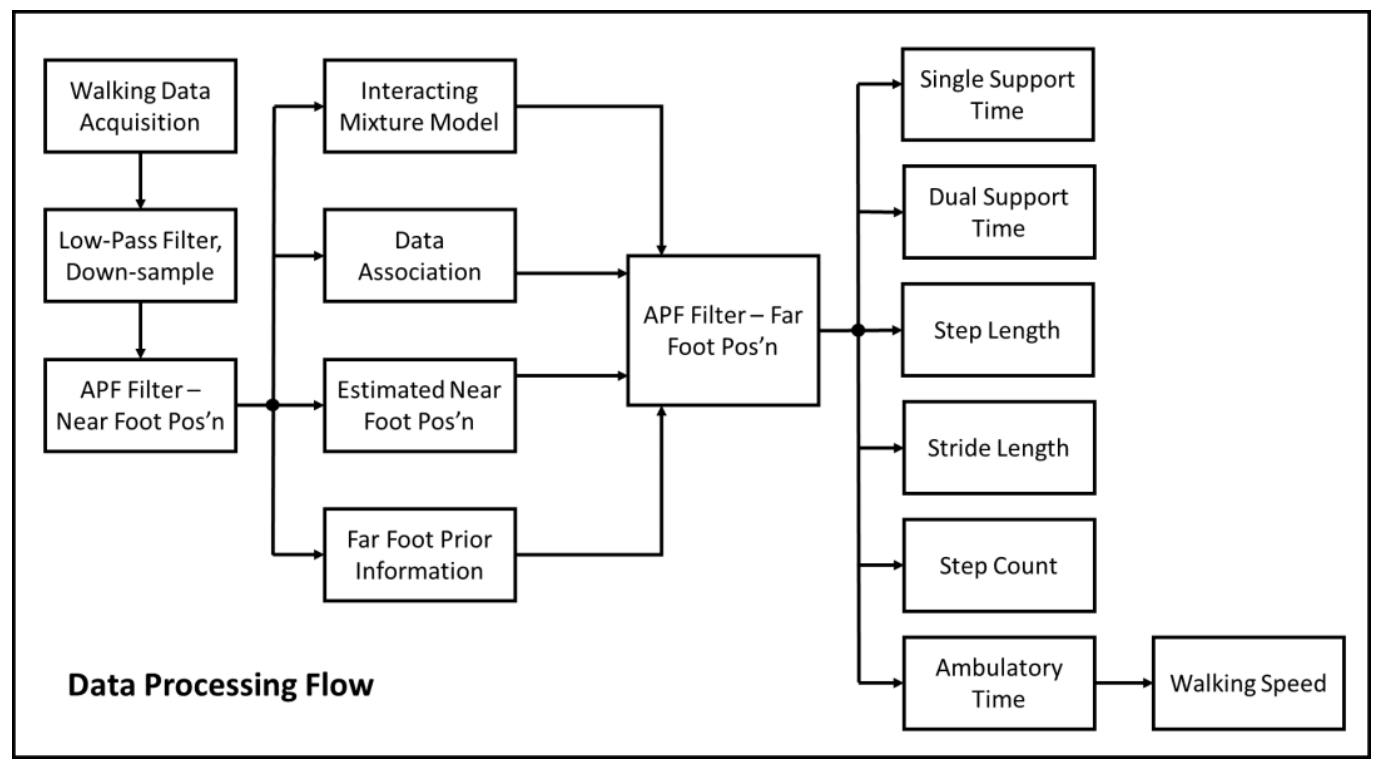

Figure 8: Flow chart of the data processing workflow. Data processing begins where data acquisition ends and proceeds until relevant gait metrics are extracted for analysis by a clinician.

fourth link from the bottom a waveform with a short spike followed by a tall spike is visible. This waveform is created by a far foot swinging followed by a near foot swinging. In the top right, the rectangle depicting the walking area a graph of ideal traces is depicted. The traces are each associated with a single link and it can be seen that the non-zero parts of the signal are exactly the same as the waveform segments above the walking rectangle. This figure is included to provide some intuition and vocabulary for the following discussion of data processing, algorithms, and analysis.

Below the ideal data figures are two images of real data. The bottom right image depicts data as a set of traces, one per sensor. Note that traces are indexed from top to bottom. The image on the bottom left depicts the same data as a heat map in which the color reflects the energy with cooler colors (blues) representing low energy, and hot colors (reds) reflecting higher energy. These types of images will be used to relate real data for the remainder of the thesis. 
It is noteworthy that the data pictured in the bottom of Figure 7 is not as clean either as the ideal data or as the data from the IR sensor system made by the other group. The noise in the data collections taken for this thesis motivated much of the algorithm design and research herein. Some noise was due to optical interference issues, as has been noted. Other noise sources could include EM interference. Initially the hardware was designed to have a protective grounding shield for the wires, however the shields were never properly attached, leading to another potential source of noise. As pleasing as it is to have an opportunity to solve this problem, it would be ideal to have a hardware system which created data as clean as the ideal data shown above. It is the goal of the research group to create a third prototype system which minimizes noise due to hardware issues.

\section{Software}

Figure 8 depicts the steps from walking data acquisition through gait feature extraction. First, walking data is acquired from the ADC into MatLab. Next the data is low-pass filtered using an $8^{\text {th }}$-order Butterworth filter and resampled to $60 \mathrm{~Hz}$ using interpolation to achieve even sample spacing and decimation to down sample. A filter, called a particle filter, is then applied to find the near foot position. From the near foot position a model of when each foot is stationary can be estimated. Data can also be associated to either the near foot or the far foot and background. These estimates are used in a second round of filtering which estimates the far foot position. Using both the far foot and near foot estimates, gait metrics such as walking speed, step length, etc. are extracted and made available for gait analysis. 


\section{Data Pre-processing}

Data is stored from the acquisition hardware as two channels of multiplexed signal and four channels of clock (one per each of 4 bits). The two channels of multiplexed signal data are de-multiplexed into 24 channels of data, each corresponding to a single IR sensor link. Each link's signal is filtered using a polyphase finite impulse response (FIR) low pass filter. Filtering is an important step to avoid aliasing which occurs when the down-sampled data contains high frequency components which are above the Nyquist frequency of the resampling step. A polyphase filter is one which treats the signal as a multiplexed signal of $\mathrm{M}$ subsequences. The signal is de-multiplexed and sent through M low-order FIR filters which each filter one of M phases of the original signal. This schema allows the signal to be filtered in parallel. Next the data is down sampled to $60 \mathrm{~Hz}$. By oversampling, low pass filtering, and down sampling high frequency noise from the hardware data acquisition system is minimized.

Data from the IR gait system is paired with data collected simultaneously by a GAITRite gait mat. The GAITRite's data is treated as ground truth, meaning that it is assumed to be correct. RMSE values presented later will reflect the difference between the ground truth and the data collected by the IR gait system. 


\begin{abstract}
Algorithms
Data analysis begins with identifying the position of the near foot. Because the near foot is nearer it generates the stronger signal of the two feet. It is an easier task to detect the near foot position. In attempts to track both feet using a particle filter it was noticed that the nearer foot was always tracked much more accurately. The assumed reason for this is that the near foot has a higher energy signal and the two feet come together and separate during a gait cycle. For these two reasons the tracking of the far foot was often derailed by the observation of the near foot. This observation led to the idea of using data association, which will be discussed later. Once detected, the near foot position can be used to separate out the part of the signal associated with the near foot from that of the far foot. By separating the two it becomes easier to track the far foot position.
\end{abstract}

To track the near foot position an Auxiliary Particle Filter (APF) is used. The particle filter was chosen through a process of empirically testing multiple state space tracking algorithms, machine learning techniques, and heuristic tracking methods. The APF was the most effective method for tracking foot position. The APF is a method of Bayesian inference, a class of algorithm, based on Bayes Theorem which can be stated as:

$$
P(A \mid B)=\frac{P(B \mid A) * P(A)}{P(B)}
$$

This equation can be thought of as relating the probability of an event $\mathrm{P}(\mathrm{A})$ to the probability of that same event $\mathrm{P}(\mathrm{A} \mid \mathrm{B})$, after more information is taken into account. As a 
concrete example, if $A \equiv$ foot position (posn) and $B \equiv$ sensor information (data) then this can be reformulated as:

$$
P(\text { posn } \mid \text { data })=\frac{P(\text { data } \mid \text { posn }) * P(\text { posn })}{P(\text { data })}
$$

With knowledge of what data the sensors will give based on a given foot position, $P($ data|posn $)$, called the measurement model in the context of a particle filter, and with knowledge of what the likelihood of that foot position $P(p o s n)$, which is called the process model in the context of a particle filter, it is possible to find the probability of a foot position given the sensor data $P($ posn $\mid$ data $)$.

A particle filter is the application of an integral approximation technique called the Monte Carlo Method to Bayesian inference. The Monte Carlo method approximates a high-dimensional definite integral by generating random samples from a probability distribution. By avoiding the curse of dimensionality, Monte Carlo methods allow for the statistical analysis of any system which can be defined as a probability. Taken a step further, a related probability distribution can be used to do importance sampling, which takes into account where the bulk of the probability is distributed and generates more random samples in that region. This technique leads to the other name by which a variety of particle filters are know: Sequential Importance Sampling, or SIS particle filters (SIS) [27].

Particle filters are a relatively new method for solving the filtering problem because they require more computation than was reasonable in the computer systems of a 
generation ago. As with all Bayesian state space tracking techniques, one of the primary benefits of the particle filter framework is its ability to integrate prior knowledge about a system with the measured output of that system to create a more robust tracker. Particle filters differ from other methods of state space tracking in that they utilize a stochastic process to progress each particle. The group of particles, defined as points in Ndimensional space, along with their weights, which can be thought of as each particle's relative importance, represents the posterior distribution of the Bayesian inference. This method is derived from Monte Carlo integration, which represents a definite integral using a stochastic process to find points on that $\mathrm{N}$-dimensional surface. In the context of a particle filter the posterior probability density can be thought of as being represented as a sum of impulses which are represented by the particles and their weights such that:

$$
\hat{p}\left(x_{n} \mid y_{0: n}\right) \equiv \sum_{i=1}^{n_{p}} w_{n}^{i} \delta\left(x_{n}-x_{n}^{i}\right)
$$

Where $\hat{p}$ is the estimated posterior PDF, $x_{n}$ is the underlying state position at time $\mathrm{n}, y_{0: n}$ are the observations from time 0 to time $\mathrm{n}, n_{p}$ is the number of particles, $w_{n}^{i}$ is the weight of each particle $\mathrm{i}$ at time $\mathrm{n}, \delta$ is the Dirac delta impulse function, and $x_{n}^{i}$ is the estimated particle position at time $\mathrm{n}$ for particle $\mathrm{i}$. The simplest particle filter, called the Sequential Importance Sampling (or SIS) particle filter suffers from a problem called degeneracy. Degeneracy is an example of the curse of dimensionality in which particles cease to be representative of the posterior probability distribution over time. There are multiple ways to solve this problem, including resampling (which leads to the SIR particle filter), the Auxiliary Particle Filter (APF) was the focus of this research. The 
APF solves the degeneracy problem by using an auxiliary variable which represents the distribution in a pre-sampling step.

Concretely, the particle filter can be described with the following pseudo code algorithm [34]:

$$
\left[\left\{x_{n}^{i}, w_{n}^{i}\right\}_{i=1}^{n_{p}}=A P F\left[\left\{x_{n-1}^{i}, w_{n-1}^{i}\right\}_{i=1}^{n_{p}}, y_{n}\right]\right.
$$

- $\quad$ For $i=1: n_{p}$ :

$$
\text { calculate } \tilde{V}_{n-1}^{i}=w_{n-1}^{i} p\left(y_{n} \mid f_{n-1}\left(x_{n-1}\right)\right)
$$

- $\quad$ normalize the weights, $v_{n}^{i-1}=\frac{\tilde{v}_{n-1}^{i}}{\sum_{i=1}^{n p} \widetilde{w}_{n}^{i}}$

- Sample the parent trajectories $j_{i}$ using weights $v_{n}^{i-1}$

- $F o r ~ i=1: n_{p}$ :

$$
\begin{gathered}
\qquad \operatorname{draw} x_{n}^{i} \sim q\left(x_{n} \mid x_{n-1}^{j^{i}}, y_{n}\right) \\
\text { calculate the unnormalized weights } \widetilde{w}_{n}^{i}=\frac{p\left(y_{n} \mid x_{n}^{i}\right) p\left(x_{n}^{i} \mid x_{n-1}^{j^{i}}\right)}{q\left(x_{n}^{i} \mid x_{0: n-1}^{j_{i}}, y_{0: n}\right)} * \frac{w_{n-1}^{j_{i}}}{v_{n-1}^{j_{i}}} \\
\bullet \quad \text { Normalize the weights, } w_{n}^{i}=\frac{\widetilde{w}_{n}^{i}}{\sum_{i=1}^{n_{p}} \widetilde{w}_{n}^{i}}
\end{gathered}
$$

In equation (1) an overview of the particle filter is given where the subscript $\mathrm{n}$ is a time step, $\mathrm{i}$ is the index of each particle. $X_{n}$ is the position of each particle in one dimension along the walking axis of the sensor array at time $\mathrm{n}$ in K-dimensional space where $\mathrm{K}$ is the number of state variables defined for the particular problem. $Y_{n}$ is the observation for each state at time $\mathrm{n}$. Equation (2) describes the process of generating an 
auxiliary variable $\tilde{V}_{n-1}^{i}$ from the weight at the previous time step and the likelihood of the observation $y_{n}$ given the observation function on the particle $x_{n}$ which is predicted from $x_{n-1}$ using a process model which predicts a future value of $\mathrm{x}$ based upon its current value. The observation function is a model of how underlying states map to observations. It takes as input the predicted underlying state at a given time step and outputs an estimation of the observation. The observation function is also called a measurement model. Equation (3) describes normalizing the weights. Equation (4) describes the presampling step which allows the APF to avoid the degeneracy problem. Equation (5) describes updating the particle trajectory. Equation (6) describes calculating the unnormalized weight $\widetilde{w}_{n}^{i}$ using the likelihood $p\left(y_{n} \mid x_{n}^{i}\right)$, the prior $p\left(x_{n}^{i} \mid x_{n-1}^{j^{i}}\right)$, and the ratio of auxiliary variable to weight variable $\frac{w_{n-1}^{j_{i}}}{v_{n-1}^{j_{i}}}$. Equation $(7)$ describes unitnormalizing the weights. A further treatment of particle filters in general and auxiliary particle filters in specific can be found in PD Moral et al. 1996 [35] and MK Pitt et al, 1999 [36].

For the purpose of implementing the APF two models must be specially fit to the data, the process model, which defines the state transitions from one hidden state, $X_{n-1}$, to the next, $X_{n}$, and the measurement model which defines the likelihood of a specific output $Y_{n}$ given a hidden state $X_{n}$ at time n. These two models fill the role of the prior $\mathrm{P}(\mathrm{A})$ and the likelihood $\mathrm{P}(\mathrm{B} \mid \mathrm{A})$ in the Bayesian framework described. For the purpose of multi-foot tracking the process model describes the position of each foot $x(n)$ at time $n$ as a random walk on previous position $\mathrm{x}(\mathrm{n}-1)$ driven by a white noise process $U_{\text {near,far }}(n)$ : 


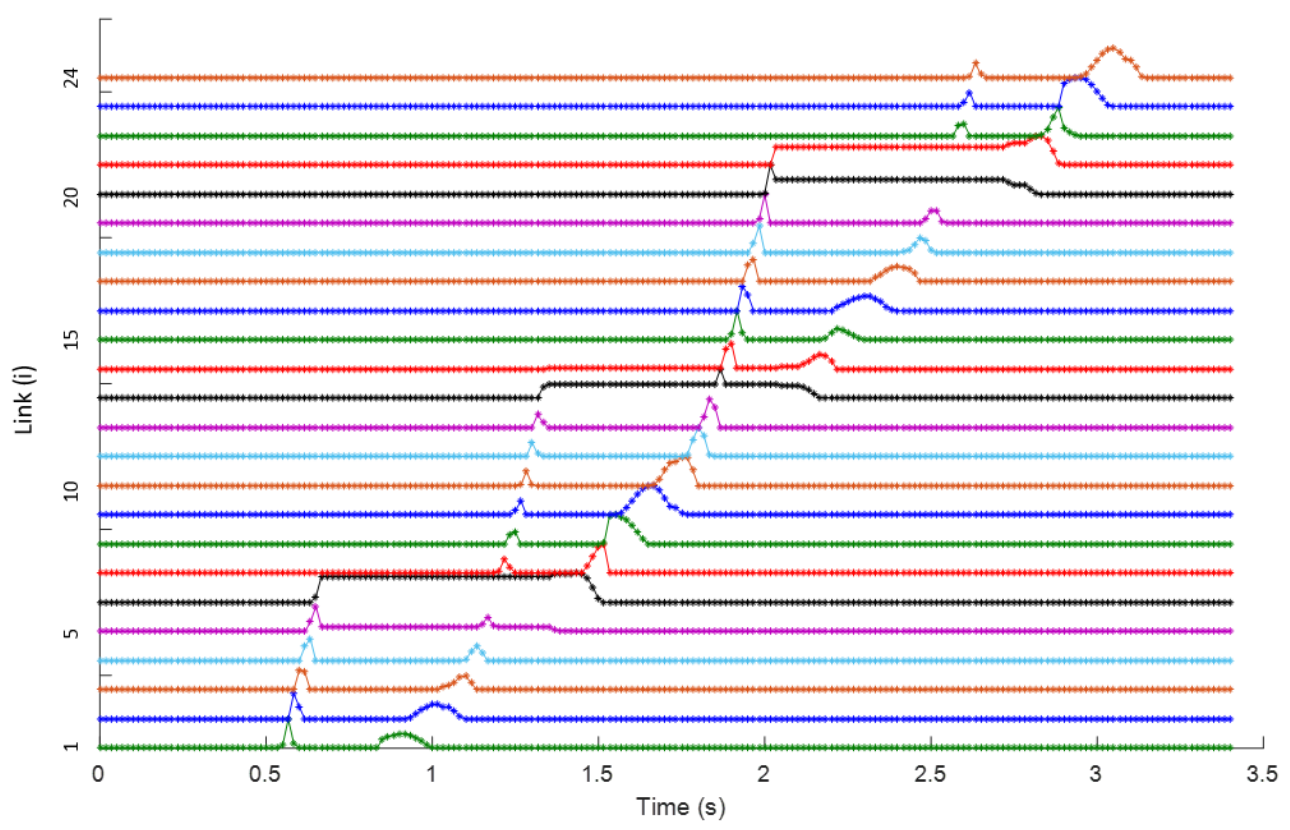

Figure 9: a visualization of Measurement Model output based upon underlying foot position for both feet. Note that the near foot is the taller waveform, beginning at $0.5 \mathrm{~s}$ while the far foot is the shorter waveform beginning around $1 \mathrm{~s}$.

$$
\begin{gathered}
\text { Process Model: } \\
X_{\text {near }}(n)=X_{\text {near }}(n-1)+U_{\text {near }}(n) \\
X_{\text {far }}(n)=X_{f a r}(n-1)+U_{f a r}(n)
\end{gathered}
$$

This process model does not capture knowledge of gait. Instead it allows the tracked object to move within a range about its current position by defining a normal distribution with mean of the estimated position and variance defined be the use case. A more advanced process model could be beneficial for future testing, however empirical testing suggested that more descriptive process models were not beneficial in implementing the particle filter. With limited time the research was shifted to focus on data association and other techniques for state space tracking. The measurement model 

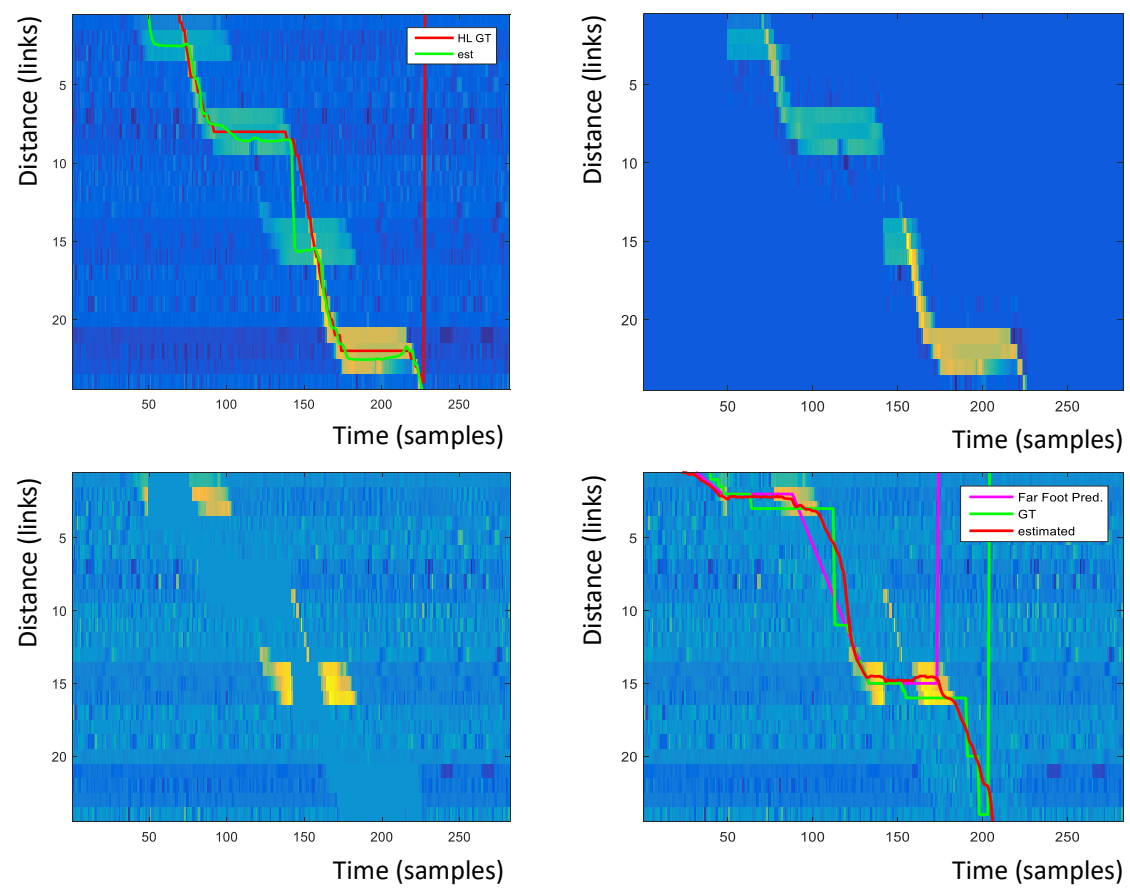

Figure 10: The three stages of data association: (a) tracking the near foot, (b) separating data windowed about the estimated near foot position from all other data, (c) tracking the far foot now that the near foot is removed. Also the creation of a prior occurs. (d) tracking the far foot (red) with expert labeling (green) and prior based upon near foot position (purple).

can be thought of as a bi-weight kernel smoother, defined as $Y_{n}=a *\left(1-X_{n}^{2}\right)^{2}$ where $\alpha$ is a scaling value. The measurement model is designed to replicate the behavior of a series of IR sensors each of which provides a smoothed peak as an object moves in front of it. The height of the peak (given by a) is proportional to the distance from the IR sensor to the object. Therefore the far foot creates a truncated peak relative to the near foot, thus requiring a smaller a term. The width of the peak is determined by the speed at which the object is traveling. For each link the measured IR value is calculated as follows:

Measurement Model:

$$
\text { For } i=1: 24 \text { : }
$$




$$
\begin{aligned}
& Y_{n}=\max \left(f_{\text {far }}\left(\Delta x_{\text {far }}(n)\right), f_{\text {near }}\left(\Delta x_{\text {near }}(n)\right)\right)+U_{n} \\
& \text { Where } f_{\text {far }}\left(\Delta x_{\text {far }}(n)\right)=\left(1-100 \Delta x_{\text {far }}^{2}\right)^{2} \text { if } \Delta x_{\text {far }} * 10<1,0 \text { else } \\
& f_{\text {near }}\left(\Delta x_{\text {near }}(n)\right)=2\left(1-100 \Delta x_{\text {near }}^{2}\right)^{2} \text { if } \Delta x_{\text {near }} * 10<1,0 \text { else }
\end{aligned}
$$

Where $\Delta X_{\text {near,far }}(n)$ is calculated for each link as the relative distance of that link from the start of the sensor array minus the estimated position of the foot. Therefore, for each link, it is a difference between foot position and sensor position. $Y_{n}$ is then the estimated measurement given the underlying state estimate. For the current sensor array there are twenty four links and therefore this measurement model is run twenty four times for each foot position. An example of the output of the measurement model for both feet can be seen in Figure 9.

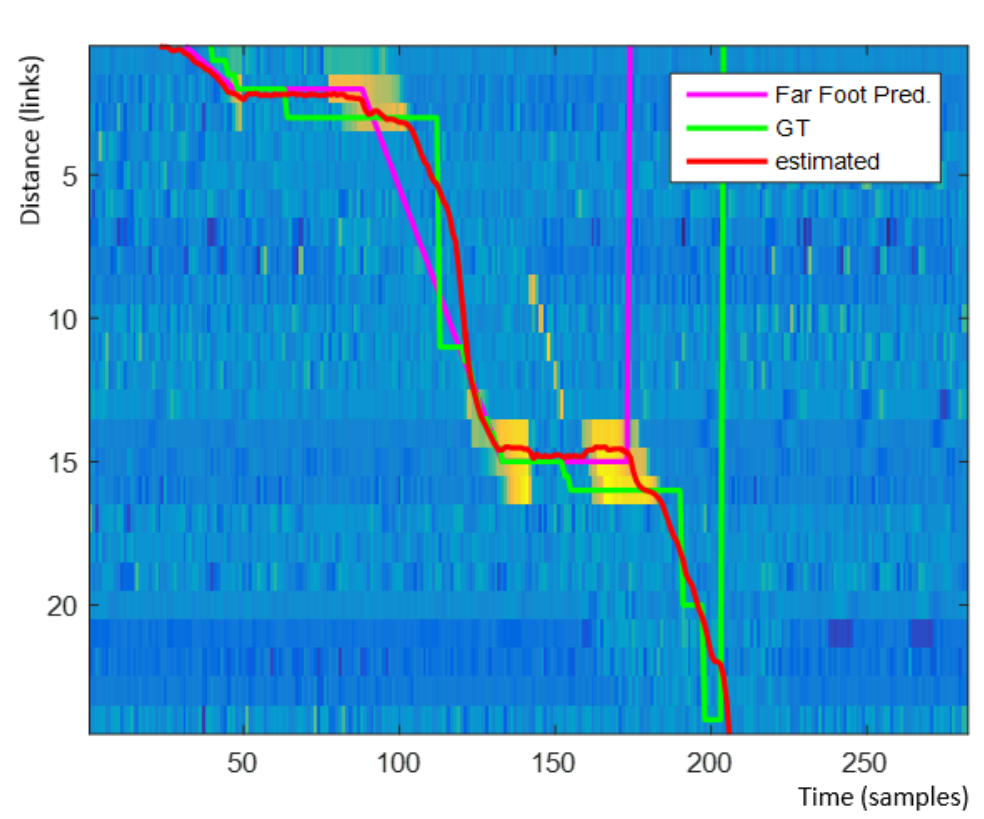

Figure 11: An example of far foot prior information (purple) overlaid on silhouette-cancelled data. Also pictured: tracked foot (red), ground truth (green). 


\section{Data Processing}

Once down sampled the data is filtered using the APF to determine near foot position as visualized in Figure 10a. As described above the APF uses a measurement model and process model which attempt to account for the position of both feet. This is due to the fact that the observations used at this stage are of both feet's data. Once the near foot position is estimated a data association method which was adapted from the multi-person motion tracking paper reviewed [19]. In their paper Adib et al. refer to the data association as sequential silhouette cancellation (SSC). This name is chosen because a silhouette of the subject being tracked is removed from the measurement data in successive steps, allowing for the remaining data to be successively filtered until all subjects are tracked. In the case of the IR gait system the object being tracked is a foot, of which there are only two, therefore a single silhouette cancelation is sufficient. The silhouette of data associated with the near foot is defined by the estimated position of the near foot from the first run of the APF Figure 10a. This data association window about time $\mathrm{n}$ is defined as a Gaussian-shaped filter (along the link axis) around the estimated position of the near foot at time $\mathrm{n}$. The Gaussian-shaped filter allows for a 'soft windowing' of the background data. The Gaussian has a mean of the estimated position at time $\mathrm{n}$ and a standard deviation of one link. A window of data defined the dot product of the Gaussian and the observation is then subtracted from the observation (Figures 10b and c) and replaced with white Gaussian noise (WGN) of the same standard deviation as noise in the data collection. Data collection noise parameters are determined when there is no subject in front of the sensor array which is defined as a time when the energy 
across all sensors is below a threshold defined as double the mean energy of the system in a walk. Because those two signals were so similar, and because the underlying states join and separate multiple times in the course of an observation, it was quite difficult to successfully track the far foot position. SSC solved the problem of having two signals which were identical except for their magnitude in the observation.

The downside of SSC, as can be seen in Figure 10 is that silhouetting the near foot data necessarily removes some of the far foot data. This results in a situation in which it is hard to track not because there is too much near foot data as before, but because there is not enough far foot data. To solve a lack of usable observation a stronger prior model is necessary (shown as the purple line in Figure 10d). Fortunately, this is an easier problem to solve because the sections of the observation from which SSC removed information are sections in which the far foot is stationary. Therefore, if those times can be identified by estimating the near foot position and using that estimate to motivate a more refined a priori model for the far foot, then the issue of data association is sufficiently resolved. This technique works specifically because there are only two objects (the feet) being tracked. A more involved technique called a joint probabilistic data association filter (JPDAF) is used extensively in computer vision research to an unknown and time varying number of subjects. The JPDAF would be an excellent avenue of future research for this project, however, due to time constraints a simpler, sufficient method was found in the SSC method.

The near foot position estimation is also used to create a strong model for positions of both feet. The model is based on a knowledge of gait. Concretely, when the 
near foot is stationary it is probable that the far foot is swinging, when the near foot is swinging it is definite that the far foot is stationary (otherwise the person is jumping). By using these two facts a strong a priori can be constructed for integration into the far foot tracking algorithm. Figure 11 shows the far foot prediction (purple), the background and far foot data (heat map), the tracked foot position (red), and the labeled foot position (green). By using a strong prior concept of the far foots position two things can be done. The first is that switching model can be constructed and the second is that a second prior can be folded into the system at the measurement model.

The near foot a priori is used to generate a Boolean value Bool ${ }_{S W I T C H}$ which switches the process model between the random walk model used in the previous APF and a stationary value:

$$
\begin{gathered}
\text { Process Model For Far Foot Tracking: } \\
\text { if } \text { Bool }_{\text {SWITCH }}={ }^{\prime} \text { moving }^{\prime}: X_{\text {far }}(n)=X_{\text {far }}(n-1)+U_{\text {far }}(n) \\
\text { if Bool } \text { SWITCH }={ }^{\prime} \text { stationary': } X_{\text {far }}(n)=X_{\text {far }}(n-1)
\end{gathered}
$$

Notice also in this process model that only the far foot is tracked. The Boolean value Bool $_{S W I T C H}$ is created using the near foots estimated location from the first execution of the APF. The estimated location is used to find the near foot position, as described in the results and discussion section, later in the thesis. The value of Bool $_{S W I T C H}$ is 'stationary' when the near foot is swinging, and 'moving' the remainder of the time. Similarly the measurement model is updated to work with the newly silhouette-canceled data: 


$$
\begin{gathered}
\text { Measurement Model For Far Foot Tracking: } \\
\text { For } i=1: 24: \\
Y_{i}^{f a r}(n)=\max \left(f_{\text {far }}\left(\Delta x_{\text {far }}(n)\right), f_{\text {far }}\left(\Delta x_{\text {prior }}(n)\right)\right)+U_{i} \\
\text { Where } f_{\text {far }}\left(\Delta x_{\text {far }}(n)\right)=\left(1-100 \Delta x_{\text {far }}^{2}(n)\right)^{2} \text { if } \Delta x_{\text {far }}(n) * 10<1,0 \text { else } \\
\text { and } f_{\text {far }}\left(\Delta x_{\text {prior }}(n)\right)=\left(1-100 \Delta x_{\text {prior }}^{2}(n)\right)^{2} \text { if } \Delta x_{\text {prior }}(n) * 10<1,0 \text { else }
\end{gathered}
$$

In the updated measurement model $X_{\text {prior }}$ is the far foot prior information. The calculation of this is described above, in the Using the updated process model and measurement model, along with the far foot prior information and the switching Boolean, an APF tracks the far foot position (Figure 10d). A better solution in this case could be a true interacting mixture model which would use multiple models running in parallel to track the feet in different parts of the gait cycle. The different models could be switched between using a mixture variable. This mixture variable could be tracked as a hidden state in the process model.

The resulting output is a position estimate for the far foot, in addition to the previously attained position estimate for the near foot. From these two position estimates gait metrics such as stride length, step count, etc. can be extracted for gait analysis. 


\section{Chapter 3: Results and Discussion}

Data was collected from four subjects of varying age, gender, height, and weight.

Subject 1 is male, early thirties, 6'2" tall, 2001bs. Subject 2 is male, early fifties, 5'9" tall, 160 lbs. Subject 3 is female, mid-twenties, 5' '" tall, 125lbs. Subject 4 is male, midforties, 5' 8" tall, 150lbs. The four subjects were asked to walk a hundred times each. Each walk consisted of traversing the IR sensor array once while stepping on the GAITRite gait mat. Data was collected simultaneously using two laptops, one as described here-in for the IR gait data, and one running the proprietary GAITRite software. Subjects were given ten seconds in which to complete the walk. Data in which the subject did not complete the walk in ten seconds, the walk was not detected by both the systems, or any other error occurred in the collection of data, was discarded. Approximately 350 usable collections were performed in this way. 99 walks taken from the first 35 walks for subjects 1-3 were then hand-labeled using a custom script in MatLab. Hand-labeled data consisted of a position estimate for the near and far foot based upon an image of the data. The green line in Figure 10d is an example of hand labeling of the far foot. The 99 hand labeled walks were used in the development and initial comparison of the analysis methods and algorithms. The

remaining approximately 250

walk were left aside for

validating the behavior of the

\begin{tabular}{|l|r|r|r|}
\hline RMSE & \multicolumn{1}{|c|}{ HMM } & Heuristic & Particle Filter \\
\hline amb time (s) & 1.014 & 0.807 & 2.040 \\
\hline walk speed $(\mathrm{cm} / \mathrm{s})$ & 20.129 & 19.746 & 21.707 \\
\hline step count & 1.520 & 1.147 & 0.652 \\
\hline stride len $(\mathrm{cm})$ & 21.558 & 51.829 & 18.237 \\
\hline step len $(\mathrm{cm})$ & 39.781 & 49.444 & 9.049 \\
\hline dual stand $(\mathrm{s})$ & 0.181 & 0.246 & 0.178 \\
\hline single stand $(\mathrm{s})$ & 0.284 & 0.264 & 0.157 \\
\hline
\end{tabular}

final analysis solution. Final

Table 3: RMSE table. Gait metrics by row, tracking algorithm by column. Algorithms include Hidden Markov Model (HMM), results are reported for the full Heuristic Model (Heuristic), and the APF model (Particle Filter). 
data set of approximately 350

walks, of which a subset of

approximated 99 walks were used

to develop the method. Data from

the GAITRite system was

\begin{tabular}{|l|r|r|r|}
\hline Norm. RMSE & HMM & Heuristic & Particle Filter \\
\hline amb time (s) & 0.357 & 0.284 & 0.719 \\
\hline walk speed (cm/s) & 22.593 & 22.163 & 24.365 \\
\hline step count & 0.371 & 0.280 & 0.159 \\
\hline stride len $(\mathrm{cm})$ & 16.259 & 39.090 & 13.755 \\
\hline step len (cm) & 61.274 & 76.157 & 13.939 \\
\hline dual stand (s) & 0.463 & 0.628 & 0.455 \\
\hline single stand (s) & 0.455 & 0.422 & 0.251 \\
\hline
\end{tabular}

considered to be the 'true state' of

Table 4: normalized RMSE values (nRMSE). Gait metrics by row, tracking algorithm by column. Algorithms include

Hidden Markov Model (HMM), Heuristic Model (Heuristic),

the walk. Thus a walking speed,

and the APF model (Particle Filter).

step count, etc. as determined by the GAITRite is the 'true' walking speed, step count,

etc. RMSE values calculated in Table 3 reflects the difference between the true value $x$

and the estimated value $\hat{x}$ for that gait metric as:

$$
X_{R M S E}=\sqrt{\sum\left(x_{n}-\hat{x}_{n}\right)^{2}}
$$

The amount of data calculated for each method is also presented in table 4. Data which cannot be estimated for a specific metric (e.g. if the APF detects only one step for a given walk, then that walk cannot have a stride length calculated) will be ignored in the calculation of RMSE for that metric. To aid understanding of the RMSE values, Table 5 will present the RMSE for each metric and each algorithm mean-normalized using the mean of the 'true' value as determined by the GAITRite:

$$
X_{N O R M}=\frac{X_{R M S E}}{\left(\frac{1}{N}\right) \sum x_{n}}
$$

Multiple gait analysis methods were investigated. These three tables will give a full understanding of the efficacy of each of the gait analysis algorithms for each gait metric. 


\section{Results}

Multiple gait analysis methods were investigated in the course of this research. The most promising methods are compared in this results section. Only the APF algorithm is described in detail in the thesis body. For a description of the other algorithms in detail please refer to The Appendix. In addition to the APF there are two other algorithms analyzed. First, the heuristic algorithm analyzes signal power to detect footfalls, and uses those footfalls to extract gait characteristics. The heuristic method is simple and effective, providing another good reference point against which to compare more complex algorithms. Second, a Hidden Markov Model (HMM) which is a state space tracking technique. The HMM uses a discrete state space to represent the same information as the process model in the context of the APF. The HMM uses the Viterbi algorithm to find an optimal path. For further description of the HMM and heuristic algorithms refer to The Appendix. Finally, the APF, that algorithm has been discussed in depth, is compared.

Development of the Heuristic algorithm was done entirely by Erich

Schafermeyer. The Auxiliary

Particle Filter was designed as part of coursework for a state space tracking course taught by

\begin{tabular}{|l|r|r|r|}
\hline Percent Data Used & \multicolumn{1}{|c|}{ HMM } & Heuristic & Particle Filter \\
\hline amb time $(\mathrm{s})$ & $48.69 \%$ & $100.00 \%$ & $90.67 \%$ \\
\hline walk speed $(\mathrm{cm} / \mathrm{s})$ & $48.69 \%$ & $100.00 \%$ & $90.67 \%$ \\
\hline step count & $100.00 \%$ & $100.00 \%$ & $100.00 \%$ \\
\hline stride len $(\mathrm{cm})$ & $76.09 \%$ & $62.68 \%$ & $100.00 \%$ \\
\hline step len $(\mathrm{cm})$ & $73.18 \%$ & $46.94 \%$ & $100.00 \%$ \\
\hline dual stand $(\mathrm{s})$ & $69.97 \%$ & $92.42 \%$ & $99.71 \%$ \\
\hline single stand $(\mathrm{s})$ & $74.64 \%$ & $95.34 \%$ & $100.00 \%$ \\
\hline
\end{tabular}

Dr. James McNames. The APF

Table 5: Percentage of data used in each calculation. Gait code was modified and adapted metrics by row, tracking algorithm by column. 


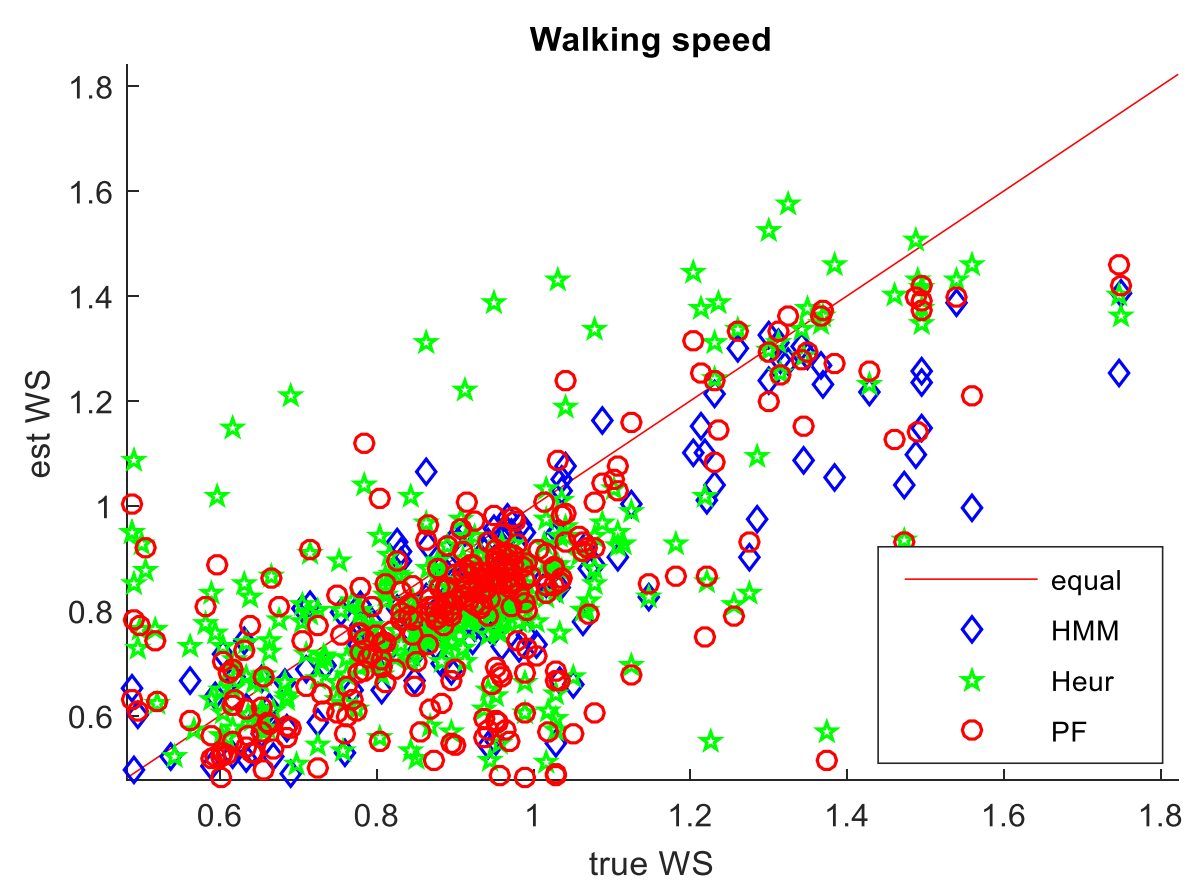

Figure 12: Scatter plot of Walking Speed (WS). True WS (as determined by GR mat) is on the x-axis, while estimated is on y-axis. Thus, the closer to the diagonal (red) line the better.

from code used in the course and presented by the professor for use in the homework assignments. Development of the Hidden Markov Model was based upon code written by Dr. Anindya Paul for MotioSens. The code was adapted by Erich Schafermeyer. The discrete state space of transition probabilities was designed by Erich Schafermeyer and Dr. Eric Wan and the emission probabilities were based on a measurement model designed by Fatema Adenwala.

\section{Stationary Foot Detection}

Before extracting individual gait metrics each foot must be determined to be swinging or stationary. A foot is considered swinging when its position stays within $7.62 \mathrm{~cm}$ of a given position for at least .33 seconds. For example if at time $12.45 \mathrm{sec}$ a foot 


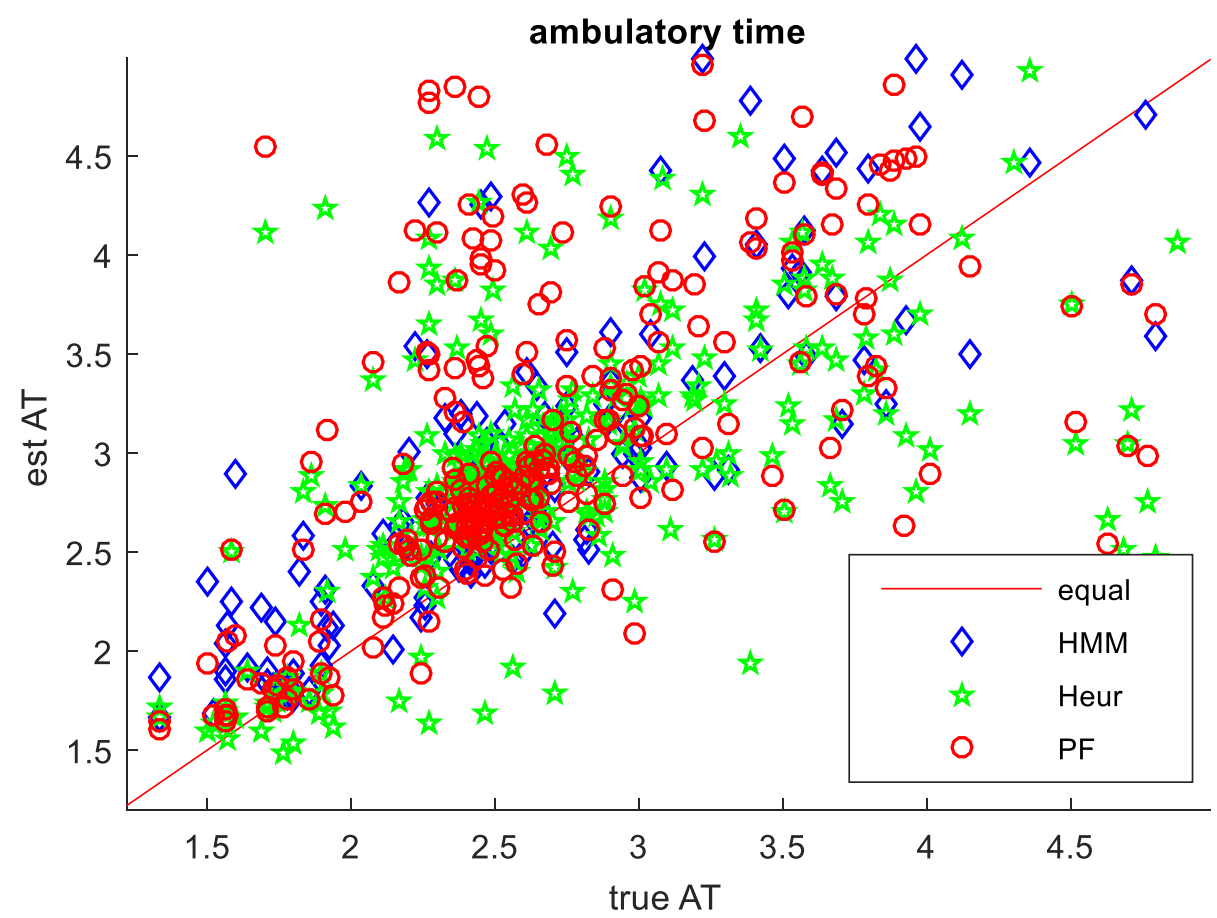

Figure 13: Scatter plot of Ambulatory Times (AT). True AT (as determined by GR mat) is on the $\mathrm{x}$-axis, while estimated is on y-axis. Thus, the closer to the diagonal (red) line the better.

is at position $1.5 \mathrm{~m}$ and at no time before $12.79 \mathrm{sec}$ does it go beyond $1.438 \mathrm{~m}$ or 1.5762

meters, then the foot is considered to be stationary in that window. If the foot stays within those bounds for a longer duration, the foot is considered stationary over that longer duration. Any time step during which a foot is in front of the sensor array and is not stationary, it is considered to be moving. Each of the tracking algorithms provides an estimated foot position for the near and far feet. The estimated positions are then found using a separate heuristic function as described here.

\section{Walking Speed}

In the data collections true walking speed ranged between $.5 \mathrm{~m} / \mathrm{s}$ and $2 \mathrm{~m} / \mathrm{s}$. The sensor system is 2.34 meters long, therefore to calculate walking speed the heuristic 
method finds the ambulatory time (AT) for each walk. AT is then used as the denominator in the formula $W S=\frac{\Delta \text { distance }}{\Delta \text { time }}$ for of walking speed (WS). The heuristic method for extracting walking speed is described below. The HMM and APF both measure walking speed in the following way. By looking at time difference between instances where both feet are at the same position (such that the ankles are crossing). Two such positions gives sufficient data for a walking speed calculation. If there are less than two such times the walk is discarded from the walking speed calculation. Otherwise it is assumed that when two feet are next to each other the torso is aligned above the feet. A linear regression, $y=m x+b$ is computed using the two or more ankle crossing times as inputs (x) and the two or more ankle crossing positions as outputs (y). The resulting slope (m) is the estimated walking speed. In Figure 12 walking speeds are depicted for each of the 3 estimation methods. The $\mathrm{x}$-axis is the ground truth walking speed as derived from the GAITRite system. The y-axis is the estimated walking speed for each respective analysis method. Therefore the closer to the $x=y$ line that a measurement is, the closer that measurement is to the true speed of the walk as measured by the GAITRite. The HMM method has performs with an RMSE of $20.1 \mathrm{~cm} / \mathrm{s}$ on $48.7 \%$ of the data, meaning that more than half of the data had no measureable AT due to the HMM not being able to measure more than one ankle crossing. The heuristic method has an RMSE of $19.7 \mathrm{~cm} / \mathrm{s}$. The heuristic method was able to detect $100 \%$ of walks Table 5. The APF has a walking speed RMSE of $21.7 \mathrm{~cm} / \mathrm{s}$ calculated on $100 \%$ of the data. Despite the fact that the HMM has a slightly better RMSE than that of the APF method, it has more than 50\% data loss Table 5. The HMM suffers from a great deal more data loss because of its use of a 
discrete state model for the gait cycle. When the HMM-based analysis system loses the objects that it is tracking, it fails to re-acquire them and the data for the entire walk is lost.

\section{Ambulatory Time}

Ambulatory time is the time spent walking during a single walk. For the heuristic analysis method ambulatory time is calculated by looking at the duration of time during which the entire system has an energy level greater than a certain threshold $\left(\mu_{P E}\right)$ :

$$
\begin{gathered}
\text { Ambulatory Time }=P E>2 \mu_{P E} \\
\text { where } P E=\sum_{l=1} x_{n, l}^{2}
\end{gathered}
$$

This value is then used to calculate walking speed. In contrast, the ambulatory time of the GR, HMM and APF are calculated based up walking speed as $A T=\frac{\text { Distance }}{W S}$ where Distance is $2.34 \mathrm{~m}$, the length of the sensor array. In Figure 13 the shorter ambulatory times, those between 1.5 seconds and 2.5 seconds are tightly grouped around the $x=y$ line. This suggests that faster walking speeds were easier for all systems to detect, perhaps because the feet made more distinct motions through the IR path. As the ambulatory time increases the measurements spread out leading to more RMSE. Comparing Figures 12 and 13 it can be seen that the walking speed RMSE seems to be tighter about the $x=y$ line for walking speeds faster than $3 \mathrm{mph}$. For reference the average walking speed of an adult on flat ground is around $3 \mathrm{mph}$. The HMM and the heuristic methods have similar AT RMSE with respective values of $1.014 \mathrm{sec}$ and 0.807 


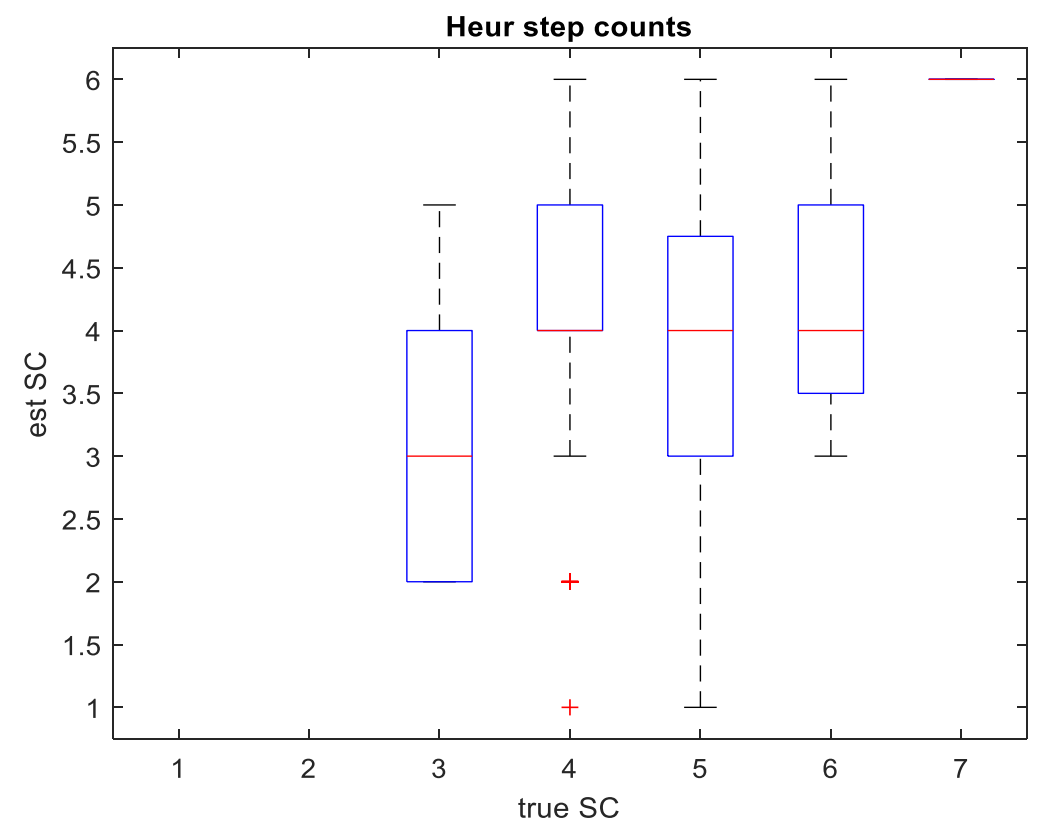

Figure 14: boxplot of step count (SC) for the heuristic algorithm. The x-axis depicts the true SC based on GAITRite mat. The y-axis depicts the estimated step count.

sec. RMSE values for ambulatory time. The APF has more than double the RMSE of the next worst method, the HMM, and both are inferior to the Heuristic method. Again the amount of data loss suggests that the HMM is not a good algorithm for AT analysis, despite its relatively low RMSE values. The HMM fails to correctly estimate ambulatory time because it is not able to track the feet with sufficient accuracy in time. This leads to foot-crossing times which are inaccurate and ultimately to an inaccurate ambulatory time estimation.

\section{Step Count}

A step is considered to be a period of $1 / 3$ of a second during which a foot is stationary. Counting all such steps leads naturally to a step count. Each column is a step 


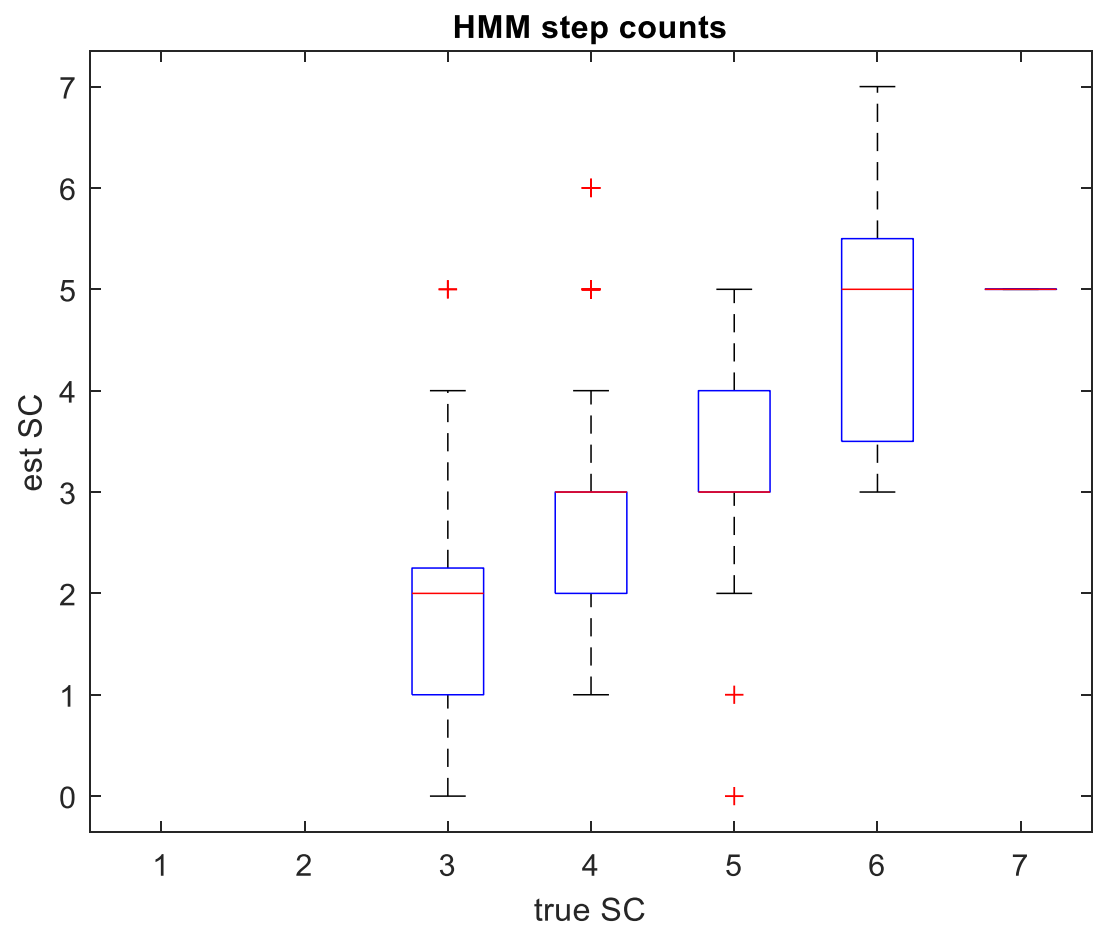

Figure 15: boxplot of step count (SC) for HMM algorithm. The x-axis depicts the true SC based on GAITRite mat. The y-axis depicts the estimated step count.

count from the GAITRite system. Each box plot is a distribution of estimated step counts as calculated by the titular method for that true step count. For example, in Figure 14 the $1^{\text {st }}$ columnar box plot depicts walks with 3 steps. The heuristic method measured step counts between 2 and 5 steps for these walks. T he median step count was 3 , the $1^{\text {st }}$ quartile is 2 , and the $4^{\text {th }}$ quartile is 4 . The $2^{\text {nd }}$ columnar boxplot depicts walks with 4 steps, and so on. For the heuristic method the mode of estimated values are equal to the true value for 3 and 4 steps, thereafter the algorithm has trouble distinguishing walks with more than 4 steps. This, again, reinforces the intuition derived from the WS and AT estimates that fewer steps (equivalent to faster walking) is easier for the system to detect. The heuristic method Figure 14 has a RMSE of 1.147 steps. The HMM method has an 


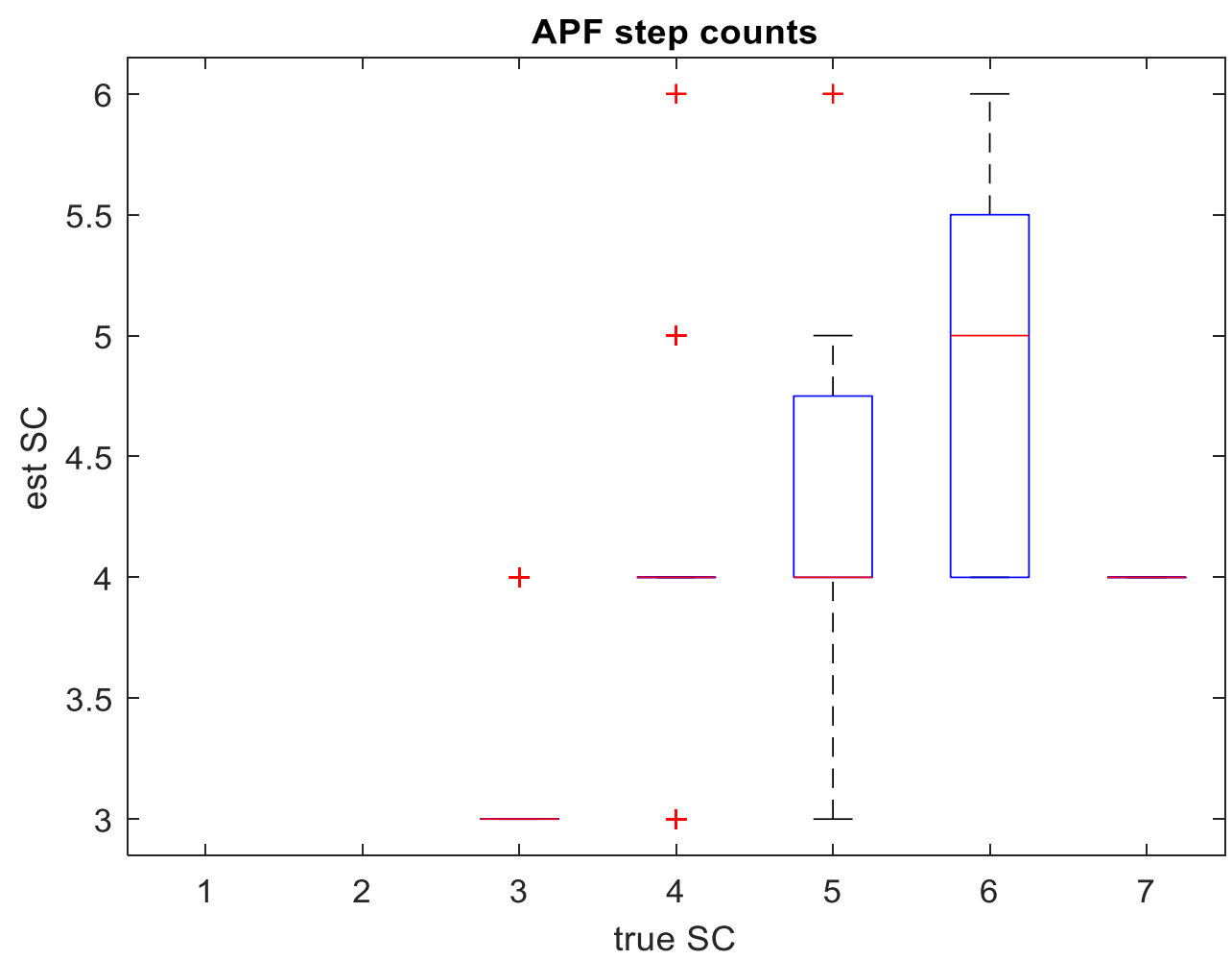

Figure 16: boxplot of step count (SC) for the APF. The x-axis depicts the true SC based on GAITRite mat. The y-axis depicts the estimated step count.

RMSE of 1.520 steps, worse than its heuristic counterpart. Looking at Figure 15 it can be seen that there is a larger deviation in the boxplot median values for true step counts. The APF in Figure 16 has an RMSE of 0.652 steps, providing the best step count estimate of any of the methods. The figure shows that the APF method is very good at estimating step count for ' 3 and 4 step walks which make up the bulk of the walks. It is less capable with walks of 5 and 6 steps. The nRMSE in Table 4 shows that the HMM and APF have fairly similar nRMSE values while the heuristic algorithm more than doubles that error. The GAITRite mat is longer than the IR sensor array by more than a meter. Steps recorded by the GAITRite are stored with position along the walking direction of the mat. 


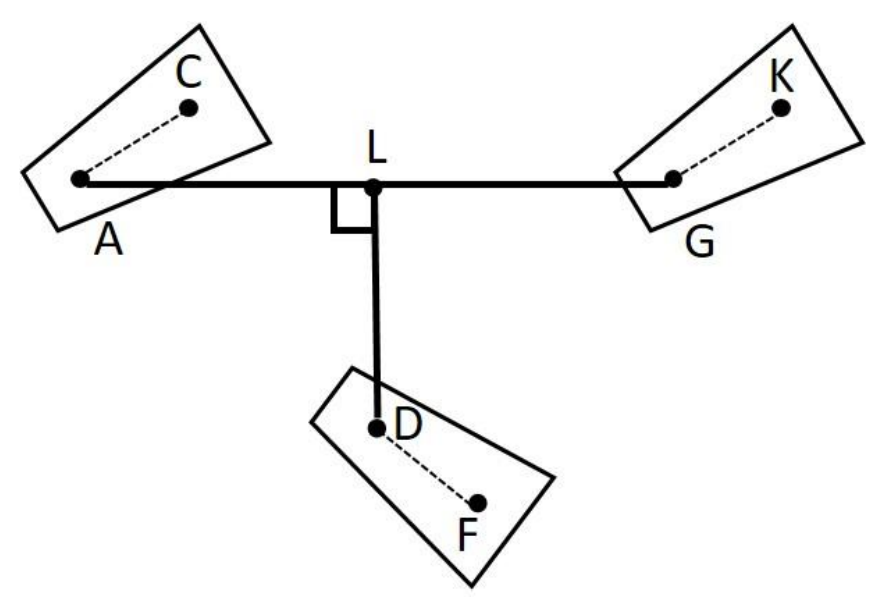

Figure 17: This figure is a reproduction of a figure from the GAITRite technical literature. Step length is measured from $\mathrm{A}$ to $\mathrm{L}$, stride length is measured from $\mathrm{A}$ to $\mathrm{G}$, and stride width is measured from $\mathrm{D}$ to $\mathrm{L}$.

To accurately record step count only those steps which occurred in front of the IR sensor array are included in the GAITRite count.

\section{Stride Length}

Stride length (STL) is calculated as the distance from on foot's placement on the ground to the place that foot next lands on the ground, visualized in (Figure 17) as line segment AG. For example in a walk the distance from where you pick your right foot up to where you set it down again is a single stride length. The HMM has a stride length RMSE of $21.6 \mathrm{~cm}$ drawn from $76.1 \%$ of data. The heuristic is the worst stride length estimator with an RMSE of $51.8 \mathrm{~cm}$ drawn from $62.7 \%$ of the data. The APF's RMSE of $18.2 \mathrm{~cm}$ which is calculated on $100 \%$ of the data is the best result both in terms of the RMSE and the amount of data calculated on. Part of the reason that the non-PF algorithms calculate their STL RMSE values on such a low volume of data is because 


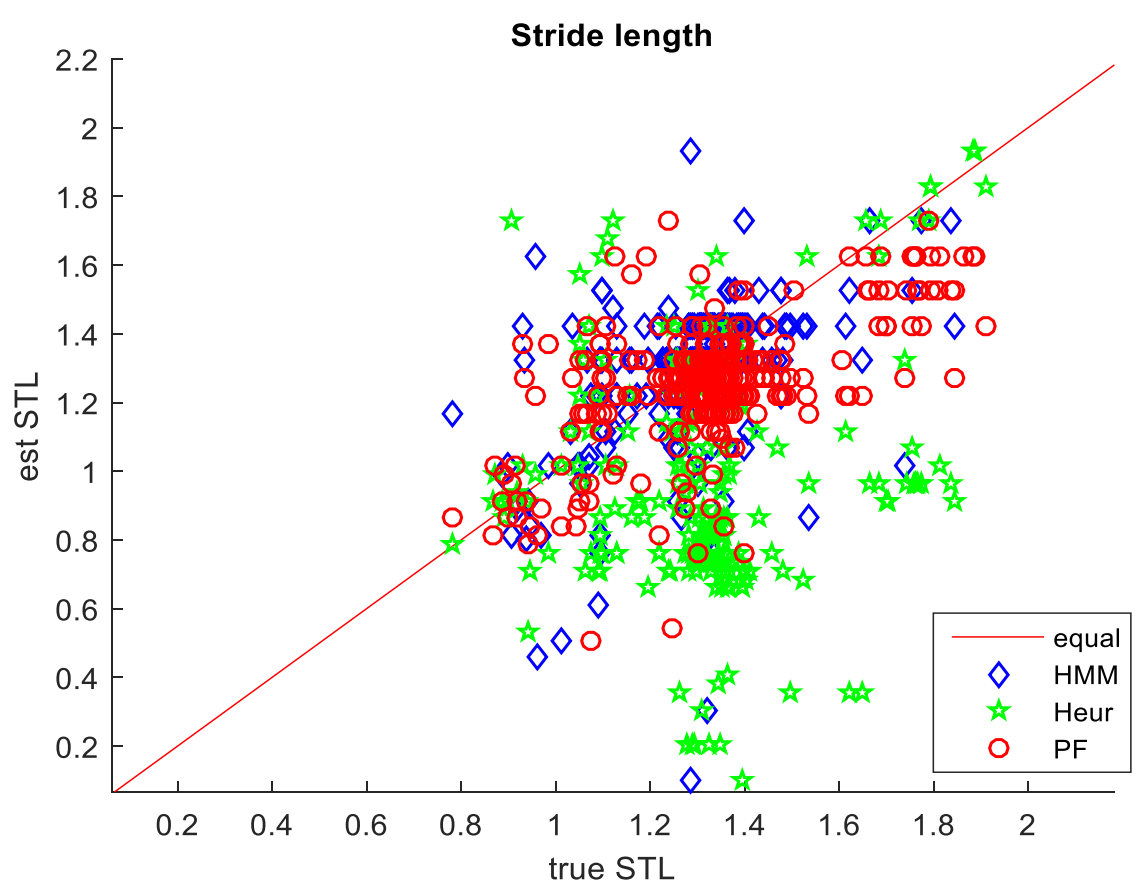

Figure 18: Stride Length (STL). The true STL is on the x-axis while the estimated STL is on the y-axis.

those algorithms had a very hard time detecting footfall. In a number of walks the number of true footfalls was only 3 , therefore a single missed footfall on the side with 2 footfalls would result in a failure to calculate stride length, while the step length is calculable with only 2 footfalls detected.

\section{Step Length}

Step Length (SL) is calculated as the distance from one foot's placement to the ground to the line tangent to the next foot placement on the ground. This is visualized in Figure 17 as the line segment AL. The HMM has an RMSE of $39.8 \mathrm{~cm}$ calculated on $73.18 \%$ of data. The fact that the percent of data calculated on is lower for step length than for stride length in the HMM method suggests that one foot was harder to detect 


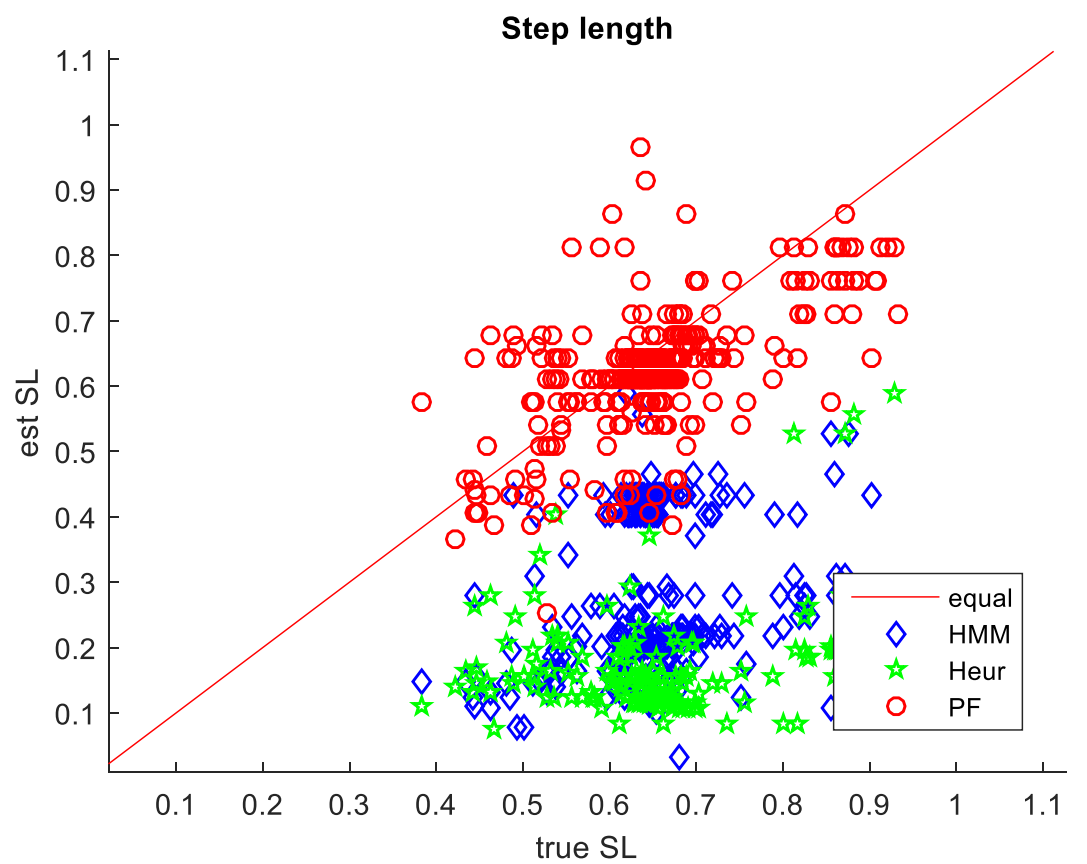

Figure 19: Step length (SL). The true SL is on the x-axis while the estimated SL is on the y-axis.

than the other, thereby making step length harder to detect than stride length. It also suggests that the estimated stride length, if differentiated between the two feet would have much higher data loss for one of the two feet (likely the far foot). The Heuristic method has an RMSE of $49.4 \mathrm{~cm}$ calculated on $46.9 \%$ of data. The particle filter has an RMSE of $9 \mathrm{~cm}$ calculated on $100 \%$ of data, and again, the lowest of the methods.

\section{Dual-Leg Standing Time}

Dual-Leg Standing Time (DST) is a measure of how much time a person stands on both legs while transitioning weight from one foot to the next during a walk. The HMM method used just under $70 \%$ of the data and had an RMSE of 0.181 seconds. The heuristic method had an RMSE of 0.246 seconds calculated on $92.4 \%$ of the data. The 


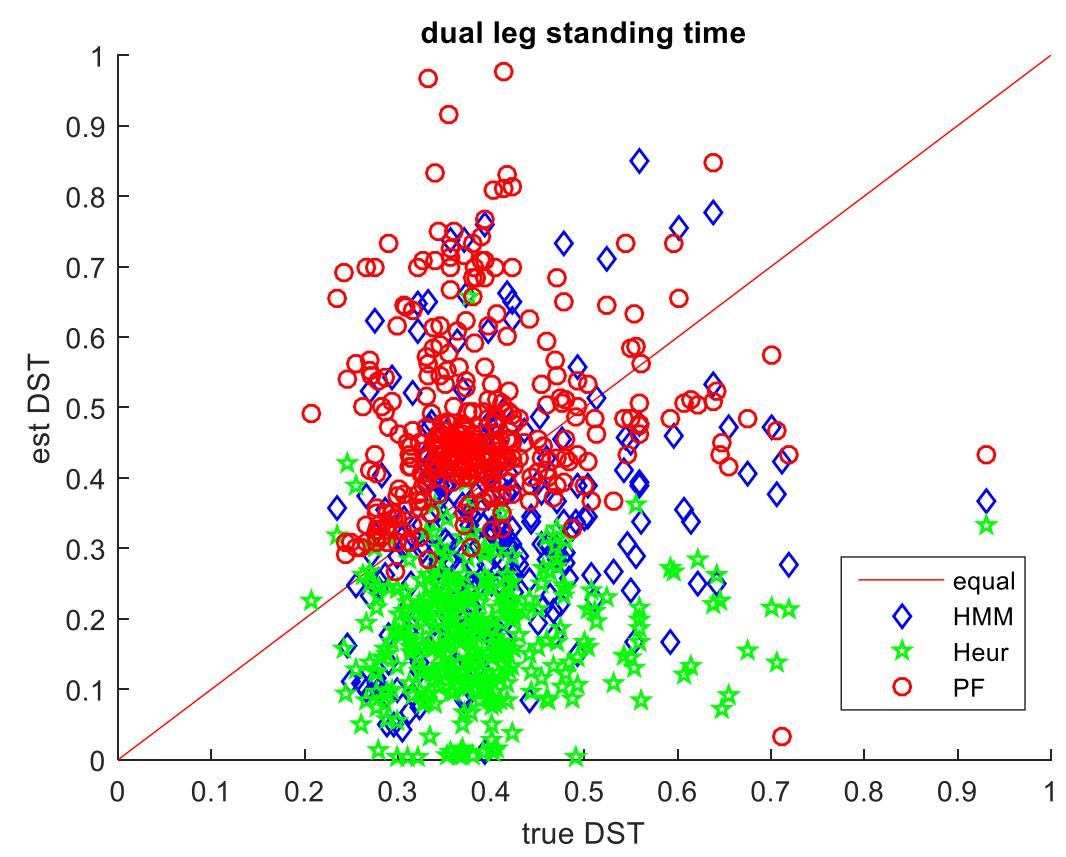

Figure 20: Dual-leg standing time (DST). The true DST is on the $\mathrm{x}$-axis while the estimated DST is on the y-axis.

APF has an RMSE of 0.178 seconds calculated on $100 \%$ of data. While RMSE is a useful measurement for many of the gait analysis metrics, the standing time values are a bit difficult to quantify because there is little natural intuition about how long a person stands on both feet during a walk. The superior measurement is then the nRMSE presented in Table 5 which shows that the APF and HMM both have an nRMSE of around 0.46 seconds and are mainly differentiated by the percentage of data used in the calculation.

\section{Single-Leg Standing Time}

Single-leg standing time (SST) is calculated as the average amount of time spent on each leg. The HMM has an RMSE of 0.284 seconds calculated on $74.6 \%$ of the data. 


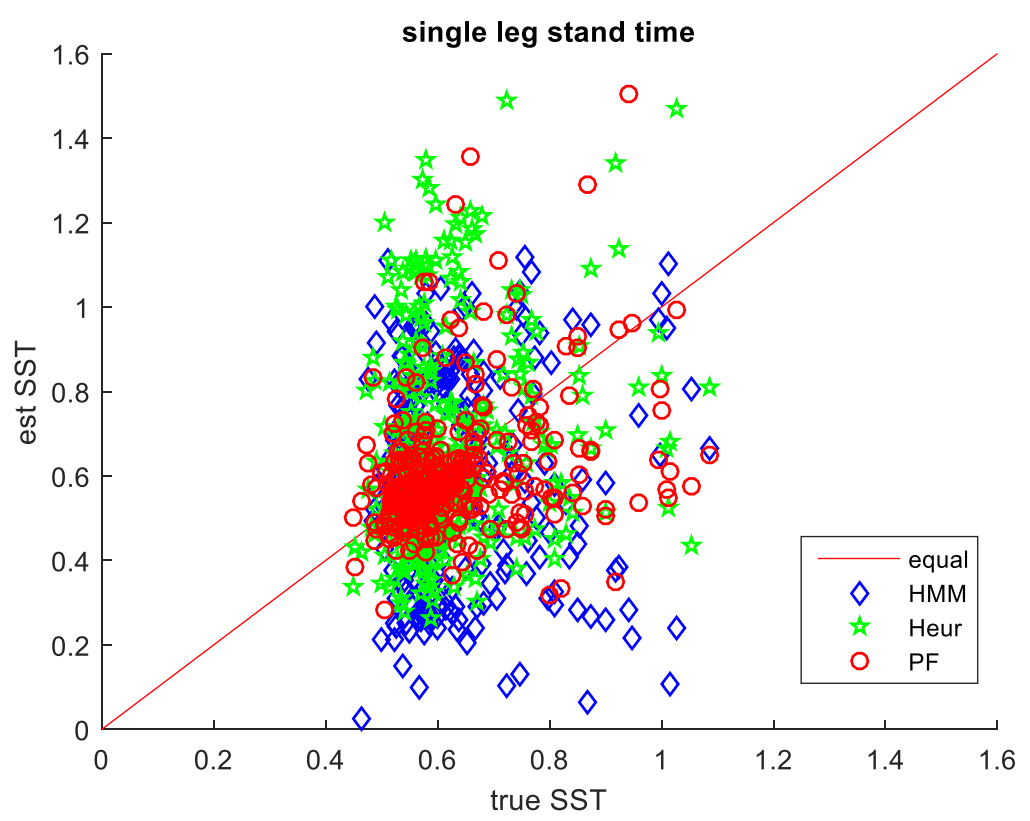

Figure 21: Single-leg standing time (SST). The true SST (as determined by the GR) is on the x-axis, while the estimated SST is on the y-axis. The closer to the diagonal line the better.

The heuristic method had an RMSE of 0.264 calculated on $95.3 \%$ of the data. The APF greatly outperformed the heuristic method with a MSE of 0.157 seconds calculated on $100 \%$ of the data. Again the nRMSE provides the greatest intuition, showing that the APF has an nRMSE of almost half that of the other two methods.

\section{Discussion}

Among the methods investigated in this thesis the Auxiliary Particle Filter is clearly the single best method for the detection and analysis of gait characteristics. The only metrics in which it was bested are the walking speed and ambulatory time. The APF calculates all of the other gait metrics with better than $99.7 \%$ data use. The AT and WS estimates are best calculated using the heuristic method which achieves both using $100 \%$ 


\begin{tabular}{|l|rrl|}
\hline Gait Metric & Lowest Accuracy Desired & Achieved Accuracy & Use Case \\
\hline Walking Speed $(\mathrm{cm} / \mathrm{s})$ & 16 & 21.7 & Stroke Recovery \\
Step Length $(\mathrm{cm})$ & 10 & 9.05 & Medication Efficacy \\
\hline Stride Length $(\mathrm{cm})$ & 5 & 18.237 & Spatial Gait Stability \\
Single Support Time (ms & 10 & 157 & Temporal Gait Stability \\
\hline Dual Support Time $(\mathrm{ms})$ & 20 & 178 & Temporal Gait Stability \\
\hline
\end{tabular}

Table 6: A Summary of Gait Metrics, their lowest desirable accuracy, and achieved results.

of data. Therefore an ideal system would incorporate the APF for gait analysis in all metrics except AT and WS for which it would use the heuristic method.

In Chapter 2 Table 2 a list of accuracies for successful gait analysis in various activities is presented. Table 6 presents the lowest necessary accuracies from Table 2 compared with the results from our IR based system. The color coding in Table 6 relates to how successfully each gait metric is estimated using the current system prototype. Achieved accuracy is calculated as RMSE. Step length, used in the context of measuring the efficacy of Parkinson's disease medication, is the sole successful measurement based upon the system. The value for walking speed is close to the desired accuracy of walking speed based upon a use case of stroke recovery monitoring, but is inaccurate by more than $35 \%$. The remaining compared metrics: stride length, single support time, and dual support time, are all significantly less accurate than they would need to be to successfully measure changes over time of spatiotemporal gait characteristics.

Comparing the results of our IR gait system with that of the other IR gait system [31] requires the introduction of a new metric which is used in the other paper. The mean absolute relative error (MARE) is calculated as: 


$$
M A R E_{x, \hat{x}}=\frac{\sum_{1}^{N}\left(\left|x_{n}-\hat{x}_{n}\right|\right)}{N} \text { where } \mathrm{N} \text { is the number of observations }
$$

The MARE is a useful metric for ignoring outliers while calculating the error between an expected outcome $\left(x_{n}\right)$ and a measured outcome $\left(\hat{x}_{n}\right)$. Fukui et al. provides only two metrics for gait analysis: walking speed and step length. In a test with elderly people, they report an error of $6.5 \%$ for walking speed and $3.8 \%$ for step length. Their results are reported from a data set consisting of 10 walks per subject with 5 male and 5 female subjects for a total of 100 walks. MARE figures are evaluated using data from an unspecified pressure mat, similar to the GAITRite, as reference values. In contrast, the IR gait system described in this paper gave an $18.5 \%$ error for walking speed and an $11 \%$ error for step length. This data is based upon approximately 350 walks collected from 4 subjects, as described previously. Clearly Fukui et al. had better results. It is this author's opinion that this analysis must be taken with a grain of salt for a few reasons. First, the results reported in Fukui et al. are based on a simple method used on a clean data set which is less noisy than the data collected from the prototype presented in this thesis, as seen in Figure 10. The data in Fukui et al. can be separated from the background using a threshold alone. This is due to a very high SNR which makes dealing with background noise easy. My suspicion is that the inferiority of our data is the result of a not fully developed hardware prototype which had improperly shielded and grounded wires. I believe that with a better hardware prototype the algorithms described in this thesis could provide better results than we have reported, and possibly better than in Fukui et al. Because the two feet are tracked independently using an APF, it would be trivial, given sufficiently high SNR, to track step width, instantaneous velocity per foot, and other 
metrics not mentioned in Fukui et al. A review of the system, assessment of its capabilities, and comments on future steps follows the next chapter which is on subject identification. 


\section{Chapter 4: Subject Identification using IR and RF Fingerprinting}

This chapter describes a passive subject identification system which is designed to be an unobtrusive, easily installed, and inexpensive solution for uniquely identifying multiple residents in a smart home. To effectively monitor biometrics of a subject living in a multi-resident home it is important to separate that subject's data from the data of caregivers, cohabitants, and visitors. For example, a male and female couple living at home with a male care giver could install these systems and the subject ID system would be used to distinguish the unique data sets.

The subject ID system described herein consists of four radio frequency (RF) transceivers and 3 infrared sensors set up in or near a doorway. Received Signal Strength (RSS) can be defined broadly as power of the signal at a time step between two transceivers. RSS from each path was used to separate three subjects, two male and one female of varying heights and weights. The testing for the subject identification hardware and the gait analysis hardware were conducted at separate times. The systems, once integrated will be able to augment each other in a number of ways. Most importantly the gait analysis system will be able to separate subjects from non-subjects. In addition the walking speed derived from the gait analysis system, as well as other features derived from the gait analysis system, could be used to perform more effective subject identification.

The system achieved $98 \%$ accuracy separating two male from one female subject and $83 \%$ accuracy separating the two male subjects from each other when using all available training data, around 2,300 observations per subject. The system maintained 
over $96 \%$ accuracy separating male subjects from female subject and $82 \%$ accuracy separating between the two male subjects when trained on only 140 observations per subject (Tables 3 and 5). This result suggests that the training data could easily be acquired within a week in a multi-resident home with minimal effort from the residents. This chapter is based on research conducted for a paper by Schafermeyer et al. published in the proceedings of the IEEE Engineering in Medicine and Biology Conference (EMBC) from August of 2015 [37].

\section{Hardware Methodology}

The system used in this research consists of 4 radio frequency (RF) transceivers operating in the $900 \mathrm{MHz}$ range at a $20 \mathrm{~Hz}$ sample rate (Figure 22). The receivers each sample a received signal strength (RSS) value which indicates how strong the signal being transmitted is upon reception. Changes in RSS are caused by objects in the room absorbing, reflecting, and blocking the signal energy. Humans, furniture, and walls can all contribute to RSS values. The transceivers used were originally designed to be used in a larger, slower sample-rate network for the

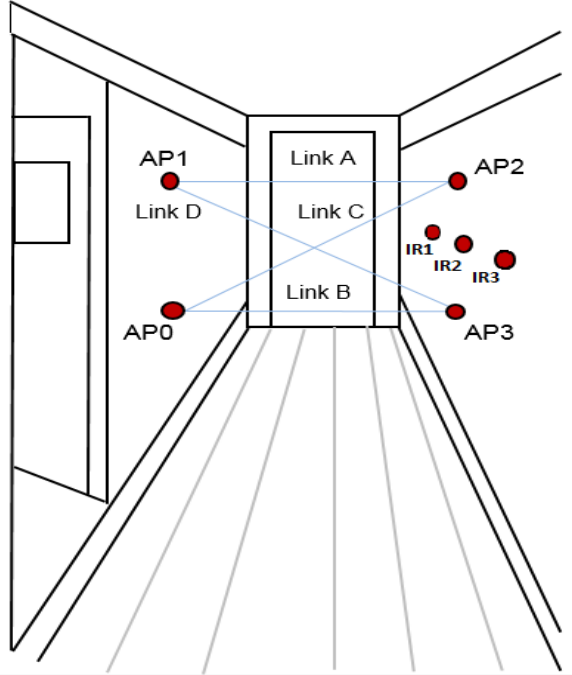

Figure 22: Access-points (AP) transceivers are arranged in a hallway. As a person walks past, the RSS disruptions between links are measured and used to recognize the person. IR sensors provide timing information to extract walking speed.

because of their low sample rate of 1 sample every 
$250 \mathrm{~ms}$, or $4 \mathrm{~Hz}$. To use them for subject ID it was necessary to run them at the significantly faster sample rate of $20 \mathrm{~Hz}$. To achieve this high rate the sensors and data collection software had to be extensively tested. It was determined that sensors from some manufacturers were not sufficiently well built to run at a higher sample rate. For this reason only a small subset of the sensors tested was usable for this data collection.

During testing sensors were mounted in a $2 \mathrm{~m}$ by $1 \mathrm{~m}$ door frame with two APs positioned at $1.75 \mathrm{~m}$ from the ground (head height) and two positioned at $1 \mathrm{~m}$ from the ground (waist height). There are three types of physical devices, or access points (AP), the first is a initiator, which sends out a signal to all three of the other access points, the second is a transceiver access point which receives then sends a signal to each of the other access points except the initiator, the final is a hub which only receives from all the other access points without transmitting. In this chapter the path between any two access points is referred to as a link. The hub receives a packet of information from each AP which contains the received signal strength (RSS) of each transmitted path between the initiator and the hub to go through that AP. An example of a single waveform for each

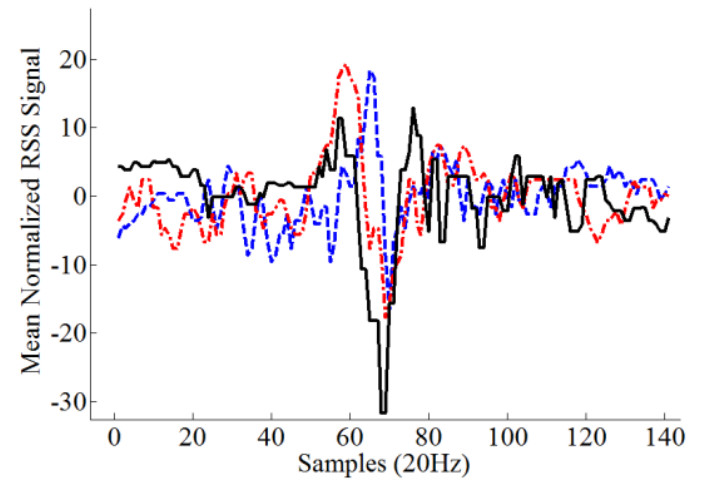

Figure 23: Example RSS waveforms for three different subjects subject is given in (Figure 23). In parallel a set of 3 infrared (IR) sensors provide a walking speed and trigger which alerts the system to a subject walking through. Because the IR triggers are at waist height (approximately $1 \mathrm{~m}$ from the ground) the trigger also negates the issue of a pet 
triggering the subject identification system. When the system is triggered a marker is placed at that point in the RF data such that it can be used to identify which subject is walking past. The physical sensors are less than one inch square and can be housed in unobtrusive plastic enclosures to be minimally invasive to a home-living environment.

The APs themselves are used for wireless transmission of packets of information. In addition, on each cycle a transmitting sensor will send a reference signal to each of its receiving sensors. The receiving sensor will be able to determine the RSS value from that reference signal. That RSS value is then added as data to the signal sent to the next sensor. The initiator transmits first and is received by access points $2,3,4$, then access points 2,3 transmit and are received by access point 4, which is the hub. Access point 4 does not transmit, it only receives. Access point 1, the initiator, does not receive, it only transmits. After transmitting each access point restarts. For each pair of sensors to transmit uninterrupted by the transmission of other sensors it is important for the transmitting sensors to have a staggered firing time from each other. It was experimentally determined that the ideal delay in firing time is around $15 \mathrm{~ms}$. This $15 \mathrm{~ms}$ value accounts for both the time for an access point which is not the initiator or the hub to receive a signal, and then to package the RSS value it has received and resends it as a part of its own packet to the next sensor in the series. The longest such path is from the initiator to AP2 to AP3 and then to AP4. This path requires 3 steps of $15 \mathrm{~ms}$ apiece, leading to the roughly $50 \mathrm{~ms}$ sample time of the sensor system as a whole.

As a person walks in the region of a transmitting and receiving sensor pair the receiver will register a change in the RSS value sent from the transmitter. This change is 
possibly due to a number of factors. One possible factor is the capacitance of the person's body absorbing some of the electromagnetic energy from that transmission, and thereby blocking that energy from reaching the receiver. Another possible factor in RSS shift during a walk is the person's body blocking or reflecting some of the RSS signal between two sensors, thereby creating a change in multi-path.

The subjects walked down a simulated corridor demarcated in the center of the BSP lab with tape. The corridor goes through a simulated door frame at its center point (Figure 22). It is important to note that during the week these data collections were being completed the lab was in active use. Students and staff worked in the lab on other projects, hardware was jostled and tables, chairs, and couches were shifted. Because of these changes the multipath signature of the room was not constant among data collections. Just as in a home living environment the features selected, as well as the algorithms used, have to be robust to these changes.

\section{Software Methodology}

The software used in this system can be broken into two parts, the data acquisition system and the data processing and analysis system. The data acquisition system used was developed specifically for this sensor set by MotioSens. It acquires data from multiple sensors at a high data acquisition rate. A reference to supporting materials for this acquisition system can be found in the appendices. Data processing and analysis was primarily conducted in MatLab. 


\section{Data acquisition}

The details of the data acquisition system are covered in depth in the appendices. The system consists of a serial interface communicating with the hub AP over a USB connection. The data acquisition system, called ERF Collector 3 can be used for programming the APs as well as collecting data from them. To program the APs each must be connected as though it were a hub and programmed directly. The network cannot be reprogrammed over the air.

Data comes into the ERF collector software and is batched until data collection is complete. It is then stored into a text file. The data collections are uniquely identified with timestamp and user ID.

\section{Algorithms for data processing}

To identify the subjects from the data collected, a method for separating among the three based upon that data must be created. A Gaussian Mixture Model (GMM) is a model which attempts to fit data given the assumption that that data can be fit using only Gaussians. Gaussian distributions are described by mean and covariance. For example a random variable $\mathrm{X}$ which is Gaussian has the probability density function:

$$
P_{X}(x)=\frac{1}{\sigma \sqrt{2 \pi}} e^{-(x-\mu)^{2} / 2 \sigma^{2}}
$$


Where $\mu$ is the mean and $\sigma^{2}$ is the covariance of the normal distribution in a multivariate GMM. A GMM is constructed of weighted sums of Gaussian distributions.

$$
\begin{gathered}
p(x \mid \lambda)=\sum_{i=1}^{M} w_{i} * g\left(x \mid \mu_{i}, \Sigma_{i}\right), \\
\text { Where } x \text { is a } \begin{array}{c}
D \text {-dimensional feature vector }, w_{i}, i=1, \ldots, \text { M are mixture } \\
\text { weights, }
\end{array} \\
g\left(x \mid \mu_{i}, \Sigma_{i}\right)=\frac{1}{(2 \pi)^{\frac{D}{2}\left|\sigma_{i}\right|^{\frac{1}{2}}} * \exp \left\{-\frac{1}{2}\left(x-\mu_{i}\right)^{\prime} \sigma_{i}^{-1}\left(x-\mu_{i}\right)\right\} \text { are the component }} \\
\text { Gaussian densities, } \\
\text { And } \lambda=\left\{w_{i}, \mu_{i}, \Sigma_{i}\right\} \text { are the parameters of Gaussian } i \\
\Sigma_{i} \text { is a diagonal covariance matrix }
\end{gathered}
$$

Gaussians are fit using the expectation maximization (EM) algorithm. EM is an iterative approach for finding the maximum a posteriori (MAP) estimate for mean and variance. The EM algorithm consists of two steps: the expectation step consists of fixing the parameters ( $\mu$ and $\sigma$ ) and solving for the posterior distribution. The maximization step consists of fixing the posterior distribution and optimizing the parameters.

Given a set of observations $x \in\left\{x_{1}, \ldots, x_{n}\right\}$, EM begins by initializing the parameters $\theta_{0} \in \Theta$. Mean initialization consisted of drawing parameters from the set of observed data at random. Standard deviation was initialized to 1. As $t \rightarrow \infty$ (until convergence):

$$
\bar{w}_{i}=\frac{1}{T} \sum_{t=1}^{T} \operatorname{Pr}\left(i \mid x_{t}, \lambda\right) \text { (weight update) }
$$




$$
\begin{gathered}
\bar{\mu}_{i}=\frac{\sum_{t=1}^{T} \operatorname{Pr}\left(i \mid x_{t}, \lambda\right) x_{t}}{\sum_{t=1}^{T} \operatorname{Pr}\left(i \mid x_{t}, \lambda\right)}(\text { mean update }) \\
\bar{\sigma}_{i}^{2}=\frac{\sum_{t=1}^{T} \operatorname{Pr}\left(i \mid x_{t}, \lambda\right) x_{t}^{2}}{\operatorname{Pr}\left(i \mid x_{t}, \lambda\right)}-\bar{\mu}_{i}^{2}(\text { diagonal covariance update })
\end{gathered}
$$

The EM steps are then repeated until convergence is reached. In this case a separate GMM is formed for data from each subject. The argmax of log likelihoods from each GMM determines the subject label.

$$
\operatorname{Pr}\left(i \mid x_{t}, \lambda\right)=\frac{w_{i} g\left(x_{t} \mid \mu_{i}, \Sigma_{i}\right)}{\sum_{k=1}^{M} w_{k} g\left(x_{t} \mid \mu_{k}, \Sigma_{k}\right)}
$$

Where $\mathrm{i}$ is the subject label.. This is the same as the maximization step in the EM algorithm. For the purposes of this research a diagonal covariance matrix was used for each Gaussian. This choice led to faster execution times but causes the model to not fit the data as well as if full covariance matrices were used. Hyper-parameters, including the number of Gaussians used and the distance metric, allow for the GMM to be adjusted to fit a specific need. Information theoretic methods can be used for identifying the appropriate number of Gaussians for each GMM. However the number of Gaussians used was found experimentally in this research.

The efficacy of the GMM algorithm was determined to exceed that of other classification methods such the heuristic approach which is described further in The Appendix. The testing method for each of these algorithms consisted of a 5-fold cross validation method in which subject data for 4 days would be used for training with the final day's data reserved for testing. Data is rotated such that 5 train/test cycles are 
completed and the results are averaged. Using k-fold cross validation avoids the risk of overfitting the training data. To ensure enough data points are used a Monte Carlo method is implemented for drawing samples from the observations.

\section{Experimental Setup}

Data was collected from 3 subjects once per day for 5 days. Each subject was asked to walk for 1 hour. A total of just over 7,000 walks were collected from 3 subjects over 5 days. Subjects varied in age, gender, height, and weight. Subjects were asked to walk at a variety of speeds, in various shoes and clothing. Each subject walked both with and without cellphones while placing calls, listening to music, or simply with the smartphone in a pocket. The lab was in active use except at the time of data collections. The system was restarted before each data collection. The three subjects consisted of one female and two males. Subject A is a 5'3" $145 \mathrm{lb}$ female, Subject B is a 5'11" $185 \mathrm{lb}$ male, and Subject C is a 6' '1" $195 \mathrm{lb}$ male. Subjects were asked to walk continuously back and forth down a $7 \mathrm{~m}$ simulated hallway constructed in the BSP lab. Each subject wore different clothing and shoes on different days. Each subject used his or her phone at some point on each day. Subjects were asked to vary gait and walking speed but gait variations were not quantified.

Further Testing for the subject identification subsystem would consist of more subjects of a wider variety of ages, sizes, weights, and gender. Because of the preliminary nature of the study done for this thesis, it was only practical to collect data on three 
subjects for the purpose of better identifying system response relating to mean-shift changes among days, the effect of changing clothing and shoes, the effect of walking with a cellphone, as well as preliminary results relating to the separation of male and female subjects. In a multi-subject smart-home environment it is likely that two or fewer subjects would be living permanently, and that other visitors would include care givers, family, and friends. A successfully implemented subject identification prototype would therefore be able to distinguish one or two subjects, for whom gait analysis was being conducted, from non-subject walkers who may be in the home for short periods of time.

Data collections taken from multiple subjects were interspersed such that data from a single subject would not be more reflective of the day it was collected on than the subject herself. Data was interspersed in the following way: each day for 5 days each of the 3 subjects walked for 1 hour. Some such differences could include changes in the position of furniture in the room, changes in the battery power of sensors, or even changes in the ambient EM noise of the room itself. A distinction is made between background data and data acquired during a walk by measuring the level of variance in the data, which increases as a subject walks by. When variance is low the data is considered to be background data and that data is averaged for each path and used for mean subtraction. By subtracting the background mean from the data it is hoped that the data becomes somewhat more stationary.

A significant amount of effort was directed towards understanding RF multipath in a multi-resident environment. Tests were conducted in three locations including an outdoor location with no hard surfaces within $20 \mathrm{~m}$ for RF signals to reflect off, an office 
environment with no furniture, only blank walls, and a fully furnished simulated apartment called the Point of Care Lab, a testing facility in the OHSU biomedical engineering department dedicated to elderly care research. It was found conclusively that multipath does exist in the latter two environments and that the largest drop in RSS does not necessarily occur when the direct path between two links is physically blocked by a body. This suggests that there are strong multipath elements to the RSS. To capture any information relevant to subject identification a broad window of time about the triggering of the sensor system should be used for data analysis.

\section{Data analysis}

Data is imported from a text file into MatLab where it is stored in matrix format. Each matrix represents a set of walks. From each of these walks a set of features must be extracted which can be used to distinguish the subjects in a meaningful way. A feature is any measurable property of the raw waveforms which can be used to distinguish one subject from another. For example the magnitude of the highest peak during a walk (Figure 23) is a feature. To find these types of features a window is first cut in time around the time that the center IR trigger is activated resulting in a windowed waveform (Figure 23). First, RSS is converted from dBs to power (Watts) by the following formula:

$$
R S S_{\text {Watts }}=10^{\frac{R S S_{p w r}}{10}}
$$

This formulation allows for the accentuation of small fluctuations in the signal. Second, a background mean subtraction is executed to remove mean-shift. Background mean is 
calculated by finding the mean of all data from a given link which has a variance below a certain threshold. This variance threshold defines the difference between foreground and background. Finally a two second window is cut from both the RSS data and the PWR data using the IR trigger. From that window a large candidate pool of features is extracted including spectral representations of the signal, mean, variance, skewness, kurtosis, peak magnitudes, number of peaks, and more.

A total of 81 candidate features were drawn from the windowed data. Using a process called sequential forward selection (SFS) [18] a set of the ten best features was selected. SFS is a fast feature selection method which chooses the best feature for a classification method by running the classifier using each feature independently. Whichever feature provides the highest classification rate is added to a permanent list of features and the remaining set is iterated over again with the permanent set included. This process is repeated until a predetermined number of features is reached or classification ceases to improve by a predetermined amount between features. The classification algorithm for SFS was a GMM. For subject identification SFS was considered complete when adding new features ceased to improve the classification of subjects. A final set of 10 features was selected. Those features include:

1. The area under the curve for the link C RSS; area is calculated as the sum of the RSS magnitude squared.

2. The first 5 principle components from the link A signal.

3. Number of unique peaks in link B RSS signal.

4. The max minus the min value of link A RSS signal.

5. Walking speed as approximated by the timing difference between IR sensor firings from IR1 and IR3.

6. The skewness of the link B RSS signal.

7. The standard deviation of the link B RSS signal. 
8. The max minus the min value of the difference between the link A and link B RSS signals.

9. The mean of link C RSS signal.

10. The difference in standard deviation between the link B RSS signal before and after the primary peak of link B RSS signal.

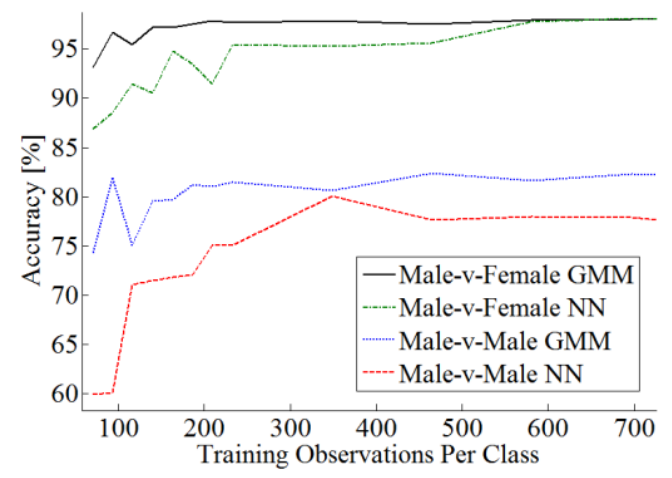

All features are scalars except for feature

Figure 24: Percent classification accuracy versus amount of labeled training data used for both a GMM 2 , which is a vector of 5 values. Features 1 and feed forward NN.

and 3 are derived from the RSS signal in dBs while features 2,4,7-10 are derived from the RSS signal in units of watts.

\section{Results and Discussion}

A GMM was found to be the most effective classification algorithm both with small amounts of input data and with large amounts. In Figure 24 a comparison was made between a feed-forward Neural Network and the GMM using varying amounts of training observations per class. The y-axis gives percent accuracy between $60 \%$ and $95 \%$. The $\mathrm{x}$ axis has the number of training observations used for classification varying from 100 to

\begin{tabular}{|c|l|l|l|}
\hline \multicolumn{1}{|r|}{$\mathbf{E}$} & Subject A & Subject B & Subject C \\
\hline Subject A & 96.97 & 1.68 & 1.35 \\
\hline Subject B & 1.76 & 85.43 & 12.81 \\
\hline Subject C & 0.58 & 20.79 & 78.64 \\
\hline
\end{tabular}

700. Four different classification tasks are

performed: 1. Separation of male and female

subjects using the GMM algorithm. 2. Separation of

Table 7: Confusion matrix of three subjects (A-female, B-male, C-male) classified using a GMM trained on all available labeled observations. 5-fold daily cross validation.

male and female subjects using the NN algorithm. 3 .

Separation of one male subject from the other male 
subject using the GMM algorithm. And 4. Separation of one male subject from the other male subject using the $\mathrm{NN}$ algorithm. The GMM outperformed the NN in both identifying male-vs-female subjects as well as identifying male-vs-male subjects. Also notable in this graph is that the GMM is able to maintain around 97\% accuracy distinguishing male-vs-female and $80 \%$ accuracy distinguishing male-vs-male subjects with only $6 \%$ of the labeled data (or around 140 samples per subject) used for training. To select 140 training samples from the training data Monte-Carlo sampling was implemented in which the original training set of around 2,300 samples was drawn from uniformly with replacement. This finding suggests that were the system to be deployed for home use; the user would only need to do around 5 minutes of walking per day for a week to train the system. Some subsequent walking would be necessary at regular intervals for the system to maintain its accuracy due to the mean-shifting of the RF as well as changes in multipath of the environment.

To evaluate performance confusion matrices for each of the three subjects were generated. Table 7 shows performance for all three subjects when the GMM is trained on $100 \%$ of the training set. In table 7 it is clear that subject $\mathrm{A}$, the female subject, is highly

\begin{tabular}{|c|c|c|c|}
\hline E & Subject A & Subject B & Subject C \\
\hline Subject A & 94.06 & 4.43 & 1.51 \\
\hline Subject B & 2.67 & 82.47 & 14.87 \\
\hline Subject C & 1.95 & 19.29 & 78.76 \\
\hline
\end{tabular}

Table 8: Confusion matrix of three subjects (A-female, B-male, C-male) classified using a GMM trained on approximately 140 labeled observations (6\% of data) per subject. 5 -fold daily cross validation with Monte-Carlo sampling. separable from subjects B, C. Subject A (female) is classified with nearly $97 \%$ accuracy, while subjects B,C are classified with $85 \%$ and $78 \%$ accuracies, respectively. The drop in classification rates is due to the more subtle height and weight difference between the two men (2", 10lbs) than between the 
men and the woman (9”, 45lbs average). Because the men and woman are of different heights and weights the APs register markedly different shifts in RSS as the subjects walk through. When the two male subjects are distinguished the difference in mass distribution is more subtle and therefore harder for the system to delineate. Table 8 shows performance for all three subjects when the GMM is trained on only $6 \%$ of the training set. The female subject is again classified with the highest accuracy at $94 \%$, while the male subjects are classified with approximately $82 \%$ and $79 \%$ accuracies. So far, analysis assumes that the classifier is evaluated on all data. If instead only the $80 \%$ of data scoring above a confidence threshold for the GMM is used, the results of training the GMM on only 140 examples approach those of using all available training data.

The research presented in this chapter represents an unobtrusive, inexpensive, and yet highly accurate system for distinguishing subjects in a multi-resident living situation. The system is implemented using sensors developed by MotioSens, a local company which specializes in tag-free localization systems. In the future further testing with more subjects in an in-home environment could be used to provide a richer data set for further algorithm development. 


\section{Contributions and Skills Developed}

This thesis represents a contribution to the fields of biomedical signal processing and elderly care in that it is an extension of known gait analysis methods to in-home use. By leveraging inexpensive electronic systems and state-of-the-art signal processing and machine learning algorithms the author was able to design and implement a prototype for automated gait analysis in a multi-resident in-home setting. This device could greatly increase the quality and quantity of data which care providers have, thereby improving their ability to make good decisions regarding patients with gait impairment.

The work completed in this paper was done in conjunction with a number of researchers at OHSU, PSU, and MotioSens. It was also supported by the help of many professors and fellow students. Specifically, prototype gait analysis system versions 1 and 3 were designed by Eric Wan, Erich Schafermeyer, and Shadman Samin. Prototype 1 was soldered and assembled by Shadman Samin with the help of Erich Schafermeyer. Prototype 3 is being constructed by Shadman Samin with the help of Madisen Phillips and Tanisha Payne. Prototype 2 was constructed by Erich Schafermeyer with the help of Shadman Samin. Software for data acquisition and pre-processing, version 1, was built by Noah Zentzis. Software version 2 was built by Erich Schafermeyer. Data collections were conducted by Eric Wan, Erich Schafermeyer, Shadman Samin, Tanisha Payne, John Condon, and Colin Doolittle. Collaboration with OHSU and the Point of Care Lab were facilitated by John Condon. Board Layout and PCB designs were done with the help of Jon Folsom. Algorithms were designed and implemented by Erich Schafermeyer, Eric Wan and Anindya Paul. 
Financial support was provided through a lab grant for elder care research to the PSU BSP Lab. This project was also supported by grant no. 1R41AG035400 from the National Institute of Aging and grant no. ETAC-12-239042 from the Alzheimer's Association. The content is solely the responsibility of the authors and does not necessarily represent the official views of the National Center for Aging or the National Institute of Health.

Skills Developed for this thesis by Erich Schafermeyer include: Machine Learning (GMM-UBM, NN, KNN, K-means, NN-HMM). Signal Processing (SIR_PF, nLMS, AR modeling, filtering, and sample theory). Hardware design and implementation: PCB design and prototyping, 2-D and 3-D fabrication techniques, soldering, gluing, welding, etc. These skills were taught by the excellent students and staff in the Maseeh College of Engineering and Computer Science. Thank you all! 


\section{Conclusion and Future Work}

The gait analysis subsystem presented in this thesis is capable of tracking and analyzing gait metrics for multiple subjects living in a smart-home environment. It can measure step length with a RMSE of $9.05 \mathrm{~cm}$, walking speed with a RMSE of $22.16 \mathrm{~cm} / \mathrm{s}$, and single leg standing time with a 157ms RMSE. A direct comparison of the three gait analysis algorithms - HMM, heuristic, and APF - shows that the auxiliary particle filter is the most effective at extracting analyzable foot positions for gait metric extraction in all cases but the ambulatory time and walking speed metrics. In those cases the heuristic method is the most effective. A future prototype of the analysis system could include a heuristic walking speed analysis method and an APF-based approach for the remaining gait metrics. It is possible that with the next hardware prototype a higher SNR will be achieved. If a higher SNR is achieved, testing with varying SNR levels of synthetic data suggest that the APF tracking could be greatly enhanced to track more states such as step width and instantaneous foot velocity as well as to track more accurately, lowering the RMSE for the gait metrics. A higher SNR could enable a greatly simplified workflow consisting of one particle filter with a more detailed process model which could potentially be used without the need for data association or a second particle filter. If a higher SNR is not achieved through better hardware improved data association techniques and a true interacting mixture model, or joint probabilistic data association (JPDAF), could be used. JPDAF is a tool used in computer vision for tracking an unknown number of targets which may interact of be obscured in a scene. 
The results of the subject identification work are highly encouraging but are preliminary. They suggest that an inexpensive, unobtrusive subject identification system could be deployed to a user's home with minimal in-home training. The system could be used in conjunction with many other biomedical applications including region level tracking systems [7], gait detection systems, drug usage systems, and more. In the case of a region level tracking system capable of tracking multiple users a subject ID system such as the one suggested in this thesis could be used to distinguish the walking paths of multiple subjects within a home environment. With this data a couple could be tracked based upon their individual statistics and relevant usage information could be communicated to each of their doctors with a high degree of certainty.

Future research on the subject ID system will focus on algorithm development. Such algorithms could include a universal background model (GMM-UBM) variant of the Gaussian Mixture Model algorithm used in this thesis. The universal background model consists of unlabeled data to which a single generic GMM can be fit. This generic GMM can then be adapted to each subject using expectation maximization. Such a model should allow for less labeled data to be used in the training of the subject identification system. The GMM-UBM system was implemented in the course of the research leading up to this thesis but was not sufficiently effective to include in the results. Its effectiveness should be as good as, or better than, the GMM alone. Future prototypes of the RF sensors in conjunction with a subject-worn tag for tracking could be used for training the system in an in-home environment. 
Both the gait analysis and subject identification subsystem have, thus far, been tested on a very small population of mostly healthy adults. Further data collections with more subjects would allow for a better idea of the efficacy of the system as a whole and could be included in a final product's data set as part of the GMM-UBM. Specifically, data collections from elderly subjects in care-facility environments could provide salient information and data sets for distinguishing gait characteristics among populations with age related diseases. 


\section{Reference}

1. Olshansky, S., et al. "Aging in America in the twenty-first century: demographic forecasts from the MacArthur Foundation Research Network on an aging society." Milbank Quarterly 87.4 (2009): 842-862.

2. SL Colby et al. Projections of the Size and Composition of the US population: 2014-2060

3. Schneider, Edward L., and Jack M. Guralnik. "The aging of America: impact on health care costs." Jama 263.17 (1990): 2335-2340.

4. The Supplement on Aging to the 1981; National Health Interview Survey Hyattsville, Md: National Center for Health Statistics; 1987. Vital and Health Statistics. Series 1. No. 21.

5. Hu, Teh-wei, Lien-fu Huang, and William S. Cartwright. "Evaluation of the costs of caring for the senile demented elderly: a pilot study." The Gerontologist 26.2 (1986): 158-163.

6. Katz, Sidney, et al. "Studies of illness in the aged: the index of ADL: a standardized measure of biological and psychosocial function." Jama 185.12 (1963): 914-919.

7. Paul, Anindya S., et al. "MobileRF: a robust device-free tracking system based on a hybrid neural network HMM classifier." Proceedings of the 2014 ACM International Joint Conference on Pervasive and Ubiquitous Computing. ACM, 2014.

8. Kaye, Jeffrey, et al. "One walk a year to 1000 within a year: Continuous in-home unobtrusive gait assessment of older adults." Gait \& posture 35.2 (2012): 197202.

9. Tilson, Julie K., et al. "Meaningful gait speed improvement during the first 60 days poststroke: minimal clinically important difference." Physical therapy 90.2 (2010): 196-208.

10. Morris, Meg E., et al. "Temporal stability of gait in Parkinson's disease." Physical Therapy 76.7 (1996): 763-777.

11. Schnall, Barri L., Brian S. Baum, and Anne M. Andrews. "Gait characteristics of a soldier with a traumatic hip disarticulation." Physical therapy 88.12 (2008): 1568-1577.

12. Wert, David M., et al. "Gait biomechanics, spatial and temporal characteristics, and the energy cost of walking in older adults with impaired mobility." Physical Therapy 90.7 (2010): 977-985.

13. Lopopolo, Rosalie B., et al. "Effect of therapeutic exercise on gait speed in community-dwelling elderly people: a meta-analysis." Physical Therapy 86.4 (2006): 520-540. 
14. Van Uden, Cornelis JT, and Marcus P. Besser. "Test-retest reliability of temporal and spatial gait characteristics measured with an instrumented walkway system (GAITRite®)." BMC Musculoskeletal Disorders 5.1 (2004): 13.

15. Muro-de-la-Herran, Alvaro, Begonya Garcia-Zapirain, and Amaia MendezZorrilla. "Gait analysis methods: An overview of wearable and non-wearable systems, highlighting clinical applications." Sensors 14.2 (2014): 33623394.Windolf, Markus, Nils Götzen, and Michael Morlock. "Systematic accuracy and precision analysis of video motion capturing systems - exemplified on the Vicon-460 system." Journal of biomechanics 41.12 (2008): 2776-2780.

16. Toulotte, C., et al. "Identification of healthy elderly fallers and non-fallers by gait analysis under dual-task conditions." Clinical rehabilitation 20.3 (2006): 269-276.

17. Cappozzo, Aurelio. "Gait analysis methodology." Human Movement Science3.1-2 (1984): 27-50.

18. Adib, Fadel, Zachary Kabelac, and Dina Katabi. "Multi-person localization via RF body reflections." 12th USENIX Symposium on Networked Systems Design and Implementation (NSDI 15). 2015.

19. Martori, Amanda Lynn. "A wearable motion analysis system to evaluate gait deviations." (2013).

20. Horak, Fay, Laurie King, and Martina Mancini. "Role of body-worn movement monitor technology for balance and gait rehabilitation." Physical therapy 95.3 (2015): 461-470.

21. Howell, David R., Louis R. Osternig, and Li-Shan Chou. "Dual-task effect on gait balance control in adolescents with concussion." Archives of physical medicine and rehabilitation 94.8 (2013): 1513-1520.

22. Clark, Ross A., et al. "Concurrent validity of the Microsoft Kinect for assessment of spatiotemporal gait variables." Journal of biomechanics 46.15 (2013): 27222725.

23. Bonnechere, Bruno, et al. "Validity and reliability of the Kinect within functional assessment activities: comparison with standard stereophotogrammetry." Gait \& posture 39.1 (2014): 593-598.

24. Stone, Erik E., and Marjorie Skubic. "Unobtrusive, continuous, in-home gait measurement using the Microsoft Kinect." IEEE Transactions on Biomedical Engineering 60.10 (2013): 2925-2932.

25. M. Montero-Odasso, M. Schapira, E.R. Soriano, M. Varela, R., Kaplan, L.A. Camera, et al. "Gait velocity as a single predictor of adverse events in healthy seniors 75 years and older” in J. Gerontol A. Biol. Sci. Med. Sci. 2005, vol. 60, pp. 1304-9. 
26. T. Buracchio, H. H. Dodge, D. Howieson, D. Wasserman, J. Kaye "The trajectory of gait speed preceding mild cognitive impairment", in Arch. Neurol., 2010 vol. 67, pp. 980-986

27. Ble, Alesandro, et al. "Executive function correlates with walking speed in older persons: the InCHIANTI study." Journal of the American Geriatrics Society 53.3 (2005): 410-415.

28. Bowen, Audrey, et al. "Dual-task effects of talking while walking on velocity and balance following a stroke." Age and Ageing 30.4 (2001): 319-323.

29. Technical literature for the GAITRite gait mat can be found at: http://www.gaitrite.com/downloads.htm

30. Fukui, Rui, et al. "Easy-to-install system for daily walking ability assessment using a distance sensor array." Journal of Ambient Intelligence and Smart Environments 7.3 (2015): 375-387.

31. Hayes, Tamara L., et al. "Unobtrusive assessment of walking speed in the home using inexpensive PIR sensors." 2009 Annual International Conference of the IEEE Engineering in Medicine and Biology Society. IEEE, 2009.

32. Austin, Daniel, et al. "On the disambiguation of passively measured in-home gait velocities from multi-person smart homes." Journal of ambient intelligence and smart environments 3.2 (2011): 165-174.

33. McNames, James. "The Auxiliary Particle Filter." State Space Tracking. Portland State University. Portland, Oregon. April 28, 2016.

34. Del Moral, Pierre. "Non-linear filtering: interacting particle resolution." Markov processes and related fields 2.4 (1996): 555-581.

35. Pitt, Michael K., and Neil Shephard. "Filtering via simulation: Auxiliary particle filters." Journal of the American statistical association 94.446 (1999): 590-599.

36. Schafermeyer, Erich R., et al. "Multi-resident identification using device-free IR and RF fingerprinting." Engineering in Medicine and Biology Society (EMBC), 2015 37th Annual International Conference of the IEEE. IEEE, 2015.

37. Bishop, C. "Pattern Recognition and Machine Learning (Information Science and Statistics), 1st edn. 2006. corr. 2nd printing edn." Springer, New York (2007). 


\section{Appendix: Algorithms for Gait Analysis and Subject ID}

Three algorithms are considered for the gait analysis portion of this research. The first algorithm considered is a heuristic approach, which uses some basic signal processing techniques to create an image map of a foot on the ground. The second is a Hidden Markov Model which uses a Bayesian framework to combine a time series of observations with an underlying model for gait. The third is a particle filter which is also a Bayesian technique for combining observations with a model. The particle filter is described in the body of the text. The two following sections address the functionality of each of the other two techniques.

\section{Gait Analysis Heuristic Algorithm}

The heuristic approach to gait analysis is quite simple. The benefits to the heuristic approach are that it does not require training data nor does it require calibration to an individual subject. The downsides are that it does not detect a foot in motion. The process begins by creating a window of data 10 seconds long. The sensors sample at $60 \mathrm{~Hz}$, and there are 24 of them, so this window is a matrix of dimension $24 \times 600$ double values. The following algorithm is then employed:

- Pre-filtering: The first step is to reduce noise using a low-pass filter implemented as a $9^{\text {th }}$ order Butterworth filter with a $3 \mathrm{db}$ cutoff at $25 \mathrm{~Hz}$. This filter is applied to each of the links independently resulting in a smoothed waveform.

- Detecting overall system energy: Summing across the 24 links for all time steps simultaneously provides a view of the overall energy of the system (below left). This energy increases as objects, such as feet, go in front of the system. A threshold can be defined as some scalar of the background energy. A walk is then defined as the anything exceeding the threshold for at least two seconds. Two
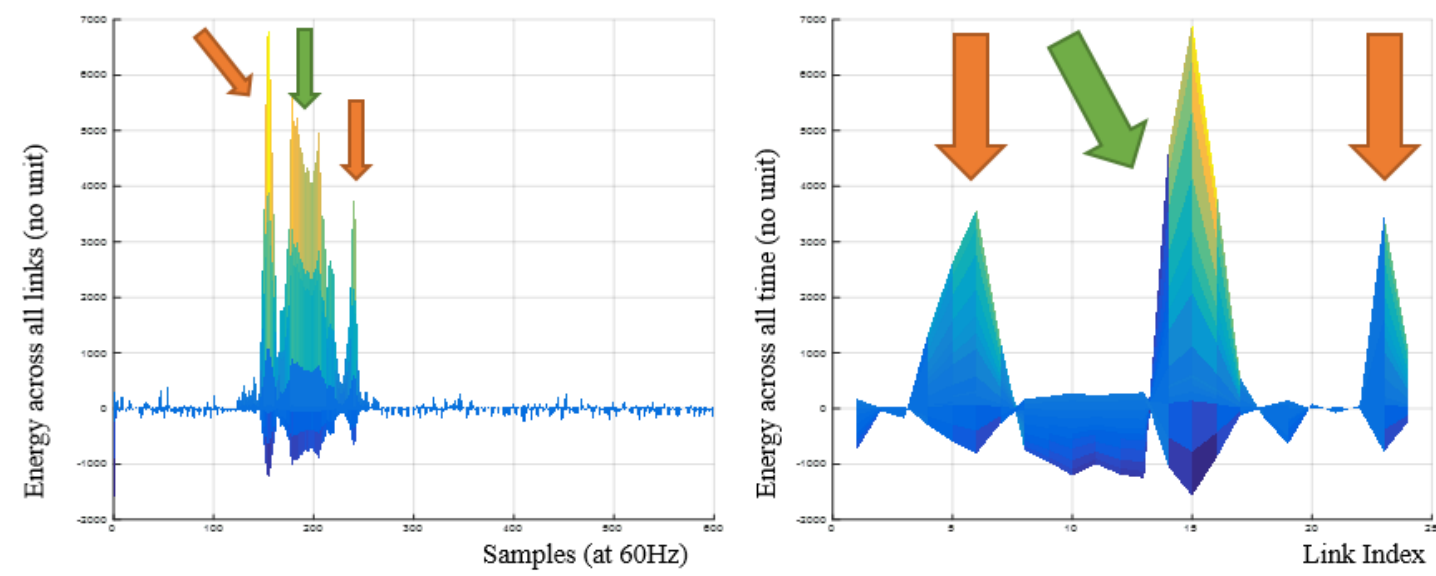

Figure: (left) Depicts the sum of energy across all links at a given sample. (right) Depicts the sum of energy across all time at a given link. 
seconds is chosen in this context because the sensor array is 7' 8 " long and a person should not walk through the system any faster than around 4 feet per second.

- Detecting Walking Speed: Walking speed can be determined directly as speed $(F P S)=\frac{\Delta \text { dist }(\text { feet })}{\Delta \text { time }(\text { seconds })}$.

- Detecting Footfall Position: As can be seen in the figure below represented by the red dotted boxes (note $A_{2}$ ): by truncating the windowed data to only include those time steps in which the total energy of the walk exceeded the threshold we gain a tighter window of data of size $24 x \mathrm{~L}$ where $\mathrm{L}<600$. To determine which links a foot fell in we then sum across all time steps, resulting in a $24 \times 1$ link-power vector. Peak detection, parameterized by a minimum peak height and a minimum distance between the peaks of at least two links, is run on the link-power vector. By making sure that peaks are at least two links apart we are able to filter for a footfall occurring in multiple links at the same time. This is essential as the foot, if longer than 7 inches will always fall in more than a single link at a time.

- Detecting Footfall Timing: As can be seen in the figure below represented by the blue boxes (note $T_{2}$ ): having detected the location of footfalls in each link it is now trivial to detect the timing of that footfall. To do so we simply look at the highest energy in the signal from each link in which a footfall was detected. A threshold is then used to determine how long the foot was on the ground.

- Creating a Footfall Map: As can be seen in the figure below represented by the black rectangles: by combining the footfall timing and position we can create a

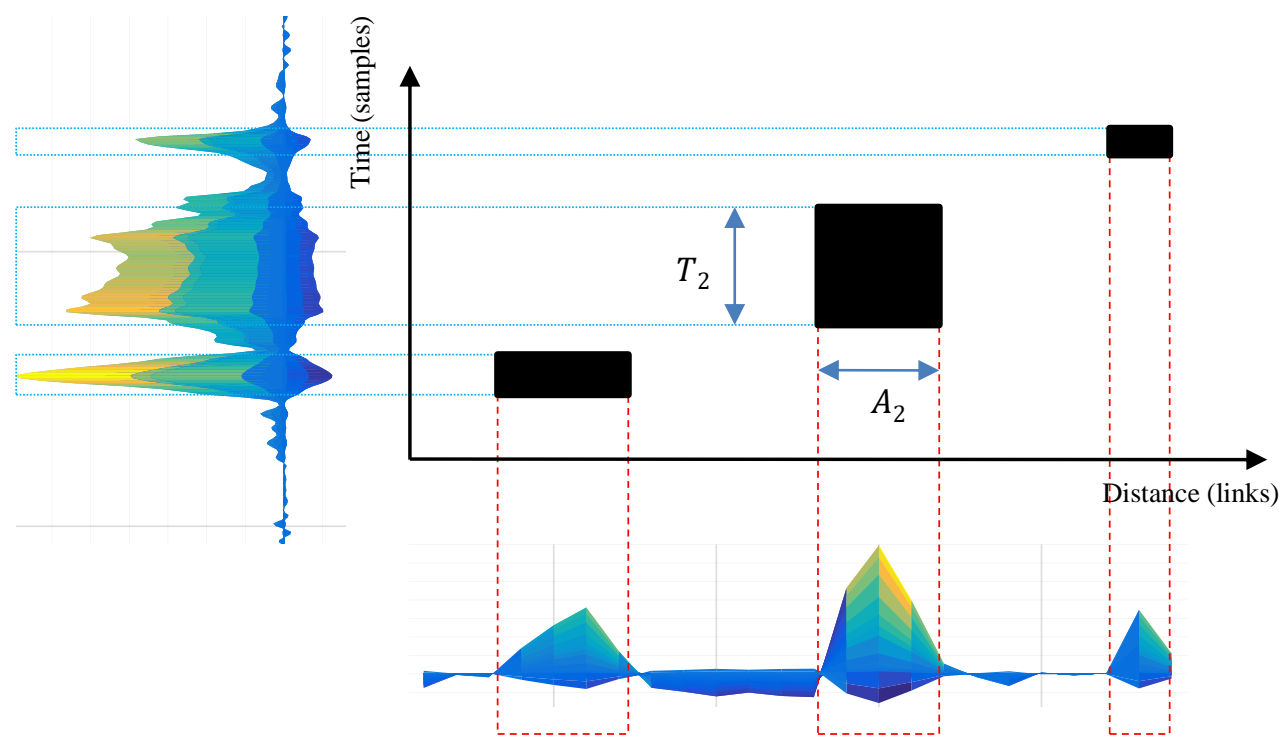

Figure: A visual depiction of how the energy graphs from the previous figure can be combined to estimate position and timing of footfalls. $T_{2}$ is the duration of the second footfall. $A_{2}$ is the duration of the second footfall. Note that the direction of the walk must be calculated first 
map of when the foot was on the ground. This map does not dictate which foot is which nor does it determine where the foot was while in the air.

\section{Hidden Markov Model}

The Hidden Markov Model (HMM) is a Bayesian technique for tracking hidden states of a system based upon a system model and measurements from the system. The HMM is very similar to the APF in respect to the parts: Bayesian inference, measurement model, process model. The HMM differs from the APF in that it uses a discrete state space. In the context of gait detection this can be thought of as the difference of the measurement of foot positions in front of a link only (HMM) versus the measurement of foot positions at any point between the first and second link, even if the foot is not directly in front of a single link (APF).

Concretely, a state sequence can be labeled $\left\{x_{1}, x_{2}, \ldots, x_{n}\right\}$ where $\mathrm{x}$ is the variable representing state and $\mathrm{n}$ is the time step. Observing state transitions for a sufficiently long period of time a conditional probability can be formed of what the next state will be based upon all previous states:

$$
p\left(x_{n} \mid x_{n-1}, x_{n-2}, \ldots, x_{1}\right) \text {. }
$$

Given a number of possible states $K$, prediction of the nth state in a sequence requires $K^{n-1}$ possible state combinations. For example, to predict the $6^{\text {th }}$ state in time in a system with 3 possible states $X=\left\{x^{1}, x^{2}, x^{3}\right\}$ would require looking at $3^{6-1}=243$ possible state combinations. To avoid huge calculations the $1^{\text {st }}$ order Markov assumption can be used, which simplifies the calculation to be:

$$
p\left(x_{n} \mid x_{n-1}, x_{n-2}, \ldots, x_{1}\right)=p\left(x_{n} \mid x_{n-1}\right) .
$$

Although the Markov assumption may not be strictly valid for the process being modeled it is often used anyway to simplify the model. In this case the Markov assumption has reduced the number of necessary state combinations from 243 to $3 * 3=9$ for all the possible combinations of $x_{n}, x_{n-1} \in X$. The probability of a sequence can be expressed using the Markov assumption as:

$$
p\left(x_{1}, \ldots, x_{n}\right)=\prod_{i=1}^{n} p\left(x_{i} \mid x_{i-1}\right)
$$

The Markov model for the states represented in the figure could also be given in a table form called the transition probability matrix (TPM). The TPM is an explicit definition of transitions made in dictionary form and used by the model to determine the next foot position with any starting state leading to a next state (in the context of the gait problem: physically the next possible foot position) with probability $p_{x_{n+1}^{i}}$ such that: 


$$
\sum_{i=1}^{K} p_{x_{n+1}^{i}}=1 \text { where } \mathrm{K} \text { is the set of all states (in this example } \mathrm{K}=3 \text { ) }
$$

The emission probability function for an HMM is defined as the probability of an observation $y_{i}$ given a state $x_{i}$ as $p\left(y_{i} \mid x_{i}\right)$. The probability of a hidden state $x_{i}$ can be found based upon knowing the previous state $x_{i-1}$ as $p\left(x_{i} \mid x_{i-1}\right)$, and the probability of an observation $y_{i}$ given a state $x_{i}$ as $p\left(y_{i} \mid x_{i}\right)$ by Bayes' rule:

$$
p\left(x_{i} \mid y_{i}\right)=\frac{p\left(y_{i} \mid x_{i}\right) * p\left(x_{i}\right)}{p\left(y_{i}\right)}
$$

Therefore the probability of $n$ events in a Markov walking sequence can be given as:

$$
p\left(x_{1}, \ldots, x_{n} \mid y_{1}, \ldots, y_{n}\right)=\frac{p\left(y_{1}, \ldots, y_{n} \mid x_{1}, \ldots, x_{n}\right) * p\left(x_{1}, \ldots, x_{n}\right)}{p\left(y_{1}, \ldots, y_{n}\right)}
$$

Using the Markov assumption and defining likelihood $L\left(x_{n} \mid y_{n}\right) \propto p\left(x_{n} \mid y_{n}\right)$ :

$$
L\left(x_{n} \mid y_{n}\right)=\prod_{i=1}^{n} p\left(y_{i} \mid x_{i}\right) * \prod_{i=1}^{n} p\left(x_{i} \mid x_{i-1}\right)
$$

Likelihood is then proportional to the probability $p\left(x_{n} \mid y_{n}\right)$.

The Viterbi algorithm is then used to find the most probable path for each state in a sequence. First, some terminology:

- Prior probabilities $\pi_{i}=P\left(x_{1}=X_{i}^{N, F}\right)$ this is the probability that the model starts in state $s_{i}$. It is fairly common to set all of these to be equal such that $\pi_{i}=$ $\frac{1}{\text { number of states }}$.

- Transition probabilities $(A)$ defined above as the probabilities of going from state i to state $\mathrm{j}: a_{i, j}=P\left(x_{n+1}=X_{i}^{N, F} \mid x_{n}=X_{j}^{N, F}\right)$.

- Emission probabilities $(B)$ also defined above as the likelihood of an observation $x$ if the model is in state $s_{i}$. because the observations in our case are continuously valued, a probability density function is defined over the observation space such that $b_{i}\left(y_{n}\right)=p\left(y_{n} \mid x_{n}=X_{i}^{N, F}\right)$.

- Model $\lambda(A, B, \pi)$ this is simply a shorthand notation so that the model can be referred to as $\lambda$ when referencing it in the description of optimization algorithms.

- Highest probability along a single path $\delta_{t}(i)=$ $\max _{\left(q_{1}, \ldots, q_{t-1}\right)} P\left[x_{1}, \ldots, x_{t}=i, Y_{1}, \ldots, Y_{t} \mid \lambda\right]$. The highest probability along a single path at time $\mathrm{t}$, which accounts for the first $\mathrm{t}$ observations and ends in state $X_{\text {final }}^{N, F}$.

This notation defines an optimal path through the HMM which takes into account both the underlying model $\lambda$ and the observed sequence $X$. The most widely used method for finding an optimal path is the Viterbi algorithm which finds a single best state sequence. The Viterbi algorithm in pseudo code as follows: 


\section{Initialization:}

- $\delta_{1}(i)=\pi_{i} b_{i}\left(\mathrm{Y}_{i}\right)$ for $1 \leq i \leq M$

- $\psi_{1}(i)=0$

Recursion:

- $\left.\delta_{t}(j)=\max _{1 \leq i \leq N}\left[\delta_{t-1}(i) * a_{i j}\right] b\left(X_{t}\right)\right]$ for $2 \leq t \leq T$

- $\psi_{t}(j)=\operatorname{argmax}_{1 \leq i \leq N}\left[\delta_{t-1}(i) * a_{i j}\right]$ for $2 \leq t \leq T, 1 \leq j \leq N$

Termination:

- $P^{*}=\max _{1 \leq i \leq N}\left[\delta_{T}(i)\right]$

- $x_{T}^{*}=\operatorname{argmax}_{1 \leq i \leq N}\left[\delta_{T}(i)\right]$

Path Backtracking:

- $x_{t}^{*}=\psi_{t+1}\left(x_{t+1}^{*}\right)$ for $t=T-1, T-2, \ldots, 1$

In the figure below it can be seen that there are $3^{N}$ possible paths through the states. To choose an optimal path at any given time step the Viterbi algorithm recursively moves to the beginning and works its way forward to find the best path given the model and observation. The final step of the Viterbi algorithm is to backtrack through the values of $\psi_{t}$ at each step to find the optimal path. For the purposes of gait tracking, the prior $(\pi)$ is set to an even probability for all foot positions entering the array within 1 meter (10 links) of the edge. The HMM can begin tracking at any step position a person with a $1 \mathrm{~m}$ or shorter stride length could take into the array. Bishop et al. [37] is an excellent resource for more information on HMMs and the Viterbi algorithm. 


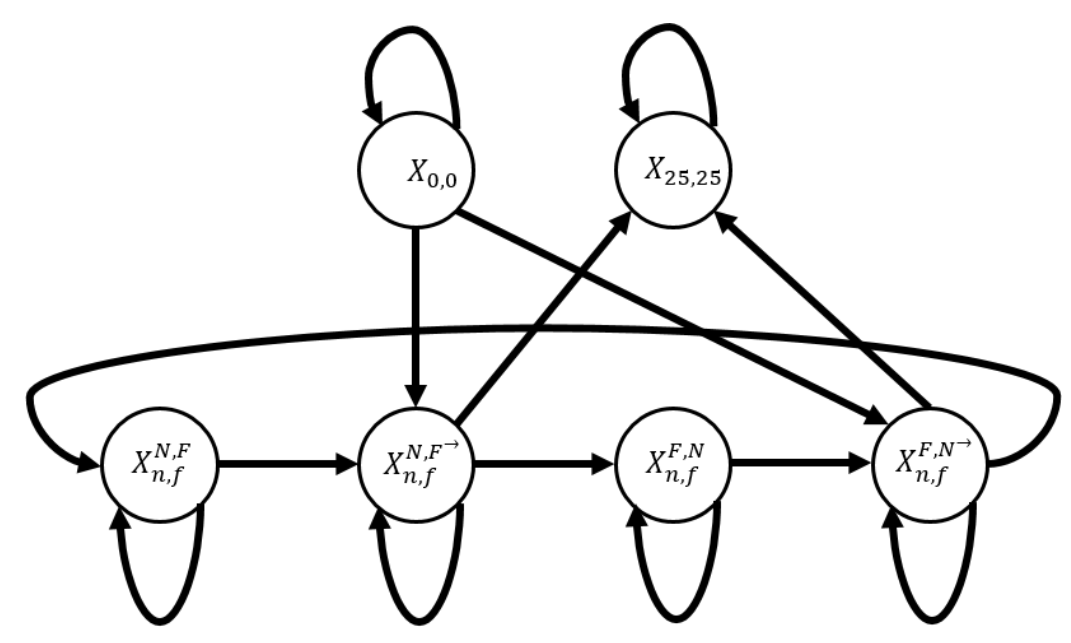

Figure: Hyper state diagram of the gait cycle. Subscripts describe which link the left and right foot are in front of. Superscripts show which foot is in motion.

The equations for the transition and the emission probabilities of the gait cycle used by the HMM are closely related to those used by the APF because both represent the same physical process. The following figure is a state space representation of the gait cycle as imagined by a discrete tracking methodology such as the HMM. In the figure on the next page the circles represent hyper-states $\mathrm{X}$ which can be thought of as generalizations of individual states. For example the state $X_{n, f}^{N, F}$ represents the set of states in which the near foot is in front of the far foot (indicated by the super-script ordering $\mathrm{N}, \mathrm{F}$ ) and both feet are stationary (indicated by the lack of a vector arrow $F^{\rightarrow}$ ). In contrast

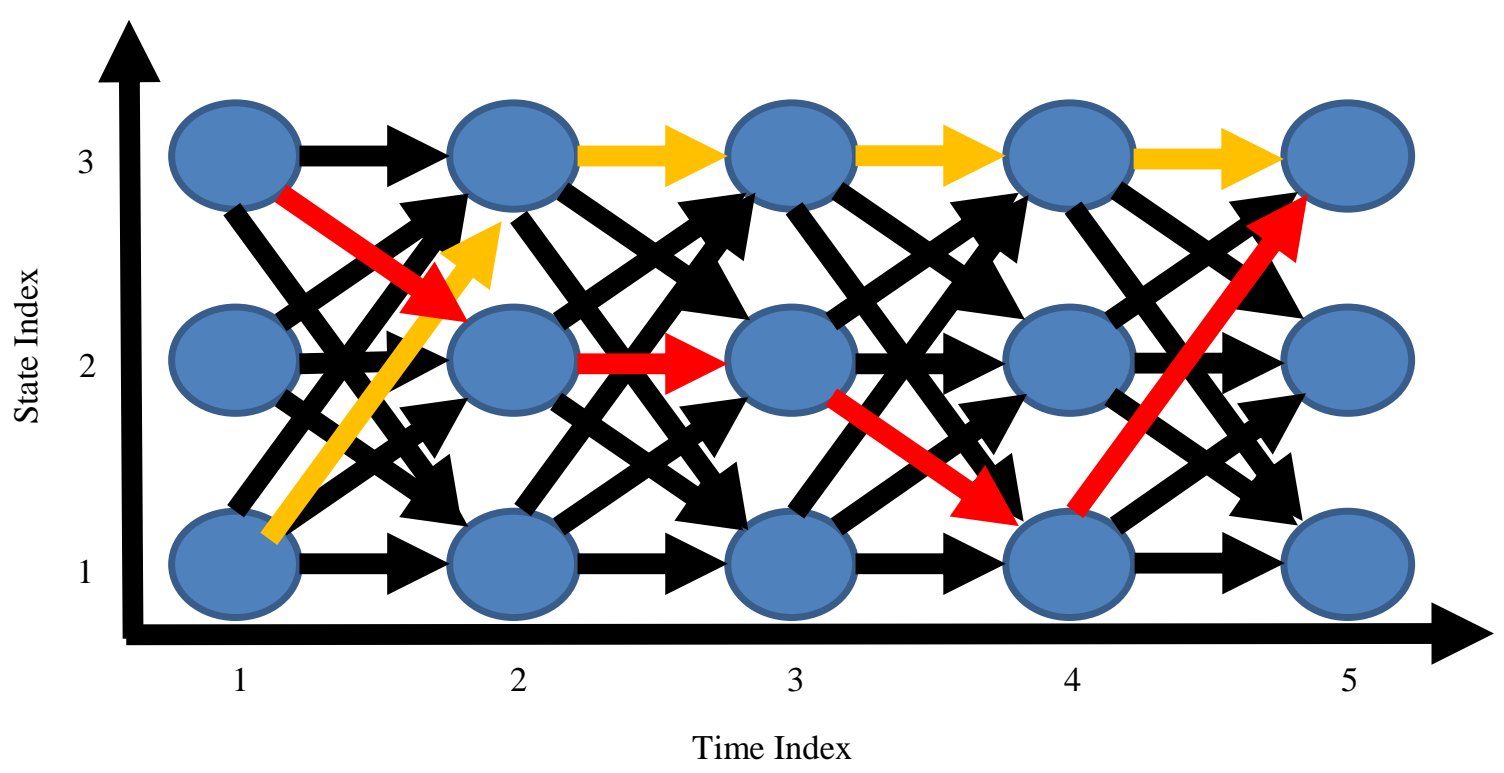

Figure: A depiction of possible paths through a state space of 3 states across 5 time steps. This figure illustrates the number of possible combinations as well as two potential individual paths through the space 
the state $X_{n, f}^{N, F^{\rightarrow}}$ represents the same ordering of feet but with the far foot in motion. The subscript indicates at which link each foot is positioned with links represented by the indices 1 through 24, the 'pre-walk' state indicated by index 0, and the 'post-walk' state indicated by index 25 . Therefore the specific state $X_{24,17}^{N, F}$ indicates that the near foot is stationary at link 24 while the far foot is in motion at link 17. That specific state is a subset of the hyper-state $X_{n, f}^{N, F^{\rightarrow}}$, therefore it can only transition to another sub-state of the same hyper-state or to one of the states contained in the hyperstate $X_{n, f}^{N, F}$ unless the feet transition out of the walkway entirely in which case the next hyper-state would be $X_{25,25}$ which is the finish state. This state can only be reached from the previous hyperstate if the specific previous state is $X_{i, 25}^{N, F^{\rightarrow}}$ where $i<25$. A visual relationship of hyper-states to foot positions can be seen in the figure on the next page. In the figure, the state $X_{n, f}^{F, N}$ is pointing at a segment of the graph in which the far foot is at link 5 and the near foot is at link 3. Therefore, the state in this interval would be: $X_{3,5}^{F, N} \in X_{n, f}^{F, N}$.

The TPM is derived from the hyper-state diagram above by careful expert labeling of all possible state transitions. There are four transition probability models: (i) for each $X_{0,0}$ and (ii) $X_{25,25}$, (iii) for $X_{n, f}^{N, F}$ and $X_{n, f}^{N, F}$, and (iv) for $X_{n, f}^{N, F^{\rightarrow}}$ and $X_{n, f}^{F, N^{\rightarrow}}$. The last two sets (iii),(iv) contain two grouped hyper states each because those hyper states behave the same way. For example the two hyper-states in (iii) are both stationary and therefore have the same modes of state transition as one another. Concretely:

i. $\quad$ For $X_{0,0}: a_{(0,0),(0,0)}=p\left(X_{0,0}, X_{0,0}\right)=p_{1}$,

$$
\begin{aligned}
& a_{(0,0),(0,1) \rightarrow}=p\left(X_{0,0}, X_{0,1}^{N, F^{\rightarrow}}\right)=\left(1-p_{1}\right)(1-\beta), \\
& a_{(0,0) \rightarrow,(1,0)}=p\left(X_{0,0}, X_{1,0}^{F, N^{\rightarrow}}\right)=\left(1-p_{1}\right) \beta
\end{aligned}
$$

Where $\beta$ is the probability of stepping with the near foot first.

ii. For $X_{25,25}: a_{(25,25),(25,25)}=p\left(X_{25,25}, X_{25,25}\right)=1$

iii. For $X_{n, f}^{N, F}: a_{(i, j),(i, j)}=p\left(X_{i, j}^{N, F}, X_{i, j}^{N, F}\right)=p_{3}$,

$$
a_{(i, j),(i, j) \rightarrow}=p\left(X_{i, j}^{N, F}, X_{i, j}^{N, F^{\rightarrow}}\right)=\left(1-p_{3}\right)
$$

Where $i, j \in\{1,25\}$ link indices. Note that the probabilities for $X_{n, f}^{F, N}$ are found by using $p_{4}$ instead.

iv. For $X_{n, f}^{N, F^{\rightarrow}}: a_{(i, j) \rightarrow,(i, j+1) \rightarrow}=p\left(X_{i, j}^{N, F^{\rightarrow}}, X_{i, j+1}^{N, F^{\rightarrow}}\right)=p_{5}$,

$$
a_{(i, j) \rightarrow,(i, j) \rightarrow}=p\left(X_{i, j}^{N, F^{\rightarrow}}, X_{i, j}^{N, F^{\rightarrow}}\right)=(1-\alpha)\left(1-p_{5}\right),
$$




$$
a_{(i, j) \rightarrow,(i, j)}=p\left(X_{i, j}^{N, F^{\rightarrow}}, X_{i, j}^{N, F}\right)=\alpha\left(1-p_{5}\right)
$$

Where $i, j \in\{1,25\}$ link indices. And $\alpha \propto|i-j|$ such that the further the two feet are apart, the more likely that the moving foot will land. Note that the probabilities for $X_{n, f}^{F, N^{\rightarrow}}$ are found by using $p_{6}$ instead.

Practically, the values $p_{1, \ldots, 6}$ must be assigned. A generic value could be assigned as a coin flip (i.e. $p_{n}=.5$ ) or could be assigned based on some knowledge of walk kinematics. In the case of this research very basic assumptions were made.

In the HMM based method, the emission probability is defined as:

$$
\begin{gathered}
b_{i}\left(y_{n}\right)=p\left(y_{n} \mid x_{n}=s_{i}\right)=\prod_{l=1}^{24} p\left(y_{n}^{l} \mid x_{n}^{l}\right) \\
p\left(y_{n}^{l} \mid x_{n}^{l}\right) \sim N\left(\mu_{l}, \sigma_{l}\right)
\end{gathered}
$$

Where $x_{n}$ is derived from $X_{n, f}^{N, F}$ and describes which foot is in front of link $l$ at time $n$. Therefore $x_{n}^{l} \in\{$ near, far, neither $\} . y_{n}^{l}$ is the sensor value at link $l$ at time $n$. Similar to $x_{n}^{l}: \mu_{l}, \sigma_{l} \in\{$ near, far, neither $\}$. The values for the mean and standard deviation $\{\mu, \sigma\}$ were found experimentally to be $\{0.58200,0.36614\}$,

$\{1.27400,0.62736\},\{-0.00766,0.08529\}$ for near foot, far foot, neither foot respectively. 
The Hidden Markov Model is a simple and powerful tool for finding hidden states using the Markov assumption. It is computationally more efficient as compared to the particle filter techniques. When used with the Viterbi algorithm the HMM based gate estimator maximizes over the entire walk, instead of simply finding the most likely next state at each time step as the APF based method does. Because a gait cycle is highly structured the Viterbi algorithm is ideal because of this path optimization. It was found experimentally that the HMM had a very hard time with low SNR data. This is because the HMM more strictly enforces the gait cycle, therefore a common failure mode of the model occurs when it gets stuck on a state and cannot transition away from it. In addition, it is less straightforward to incorporate multiple sources of prior information in an HMM than in an APF. For these reasons the HMM is unable to track subject gait with sufficient accuracy for effective gait analysis. In conclusion, possible future steps for research within the framework of the HMM algorithm could include mixing the HMM and APF approaches by leveraging an interacting mixture model to estimate the point in the gait

cycle and switch between models as a foot is moving versus when it is stationary.

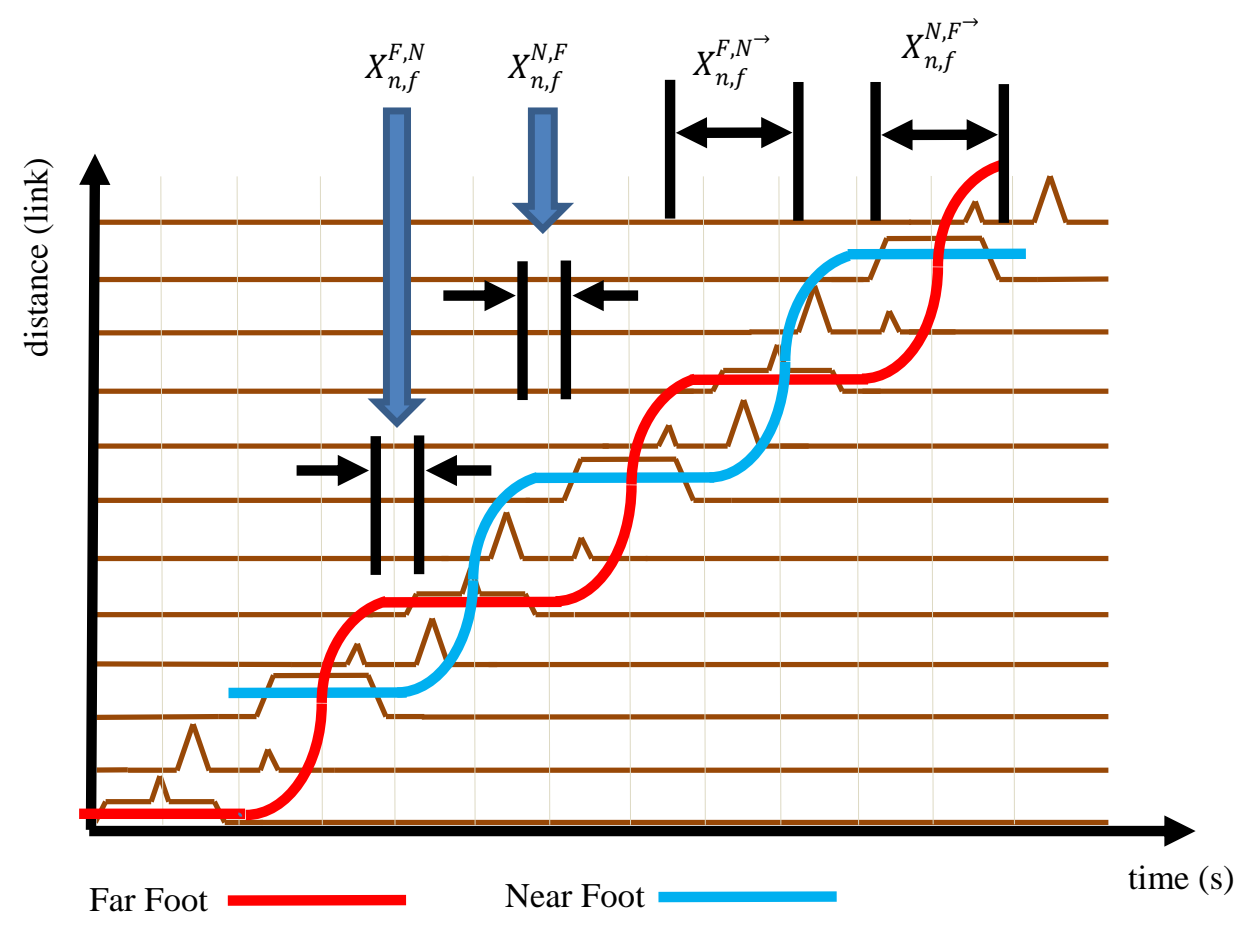

Figure: An idealized waveform for each of 12 links with an overlaid foot position for near (red) and far (blue) feet. Note the definition of the 4 hyper-states as they relate to the foot position. 